Fig S1. Tree growth and survival association curves with nitrogen and sulfur deposition by species. Each curve represents the annual growth (top graphs) or 10 year survival (bottom graphs) associations with nitrogen (N; left panels) or sulfur ( $\mathrm{S}$, right panels) deposition across the conterminous United States. Sample sizes and Pearson's correlation coefficient between N and S deposition for growth and survival analyses are given above the upper and lower panel respectively. Shaded areas represent the 2 factor support interval for the response curves. 


\section{Pacific silver fir}

$\mathbf{N}$ deposition

Abies amabilis

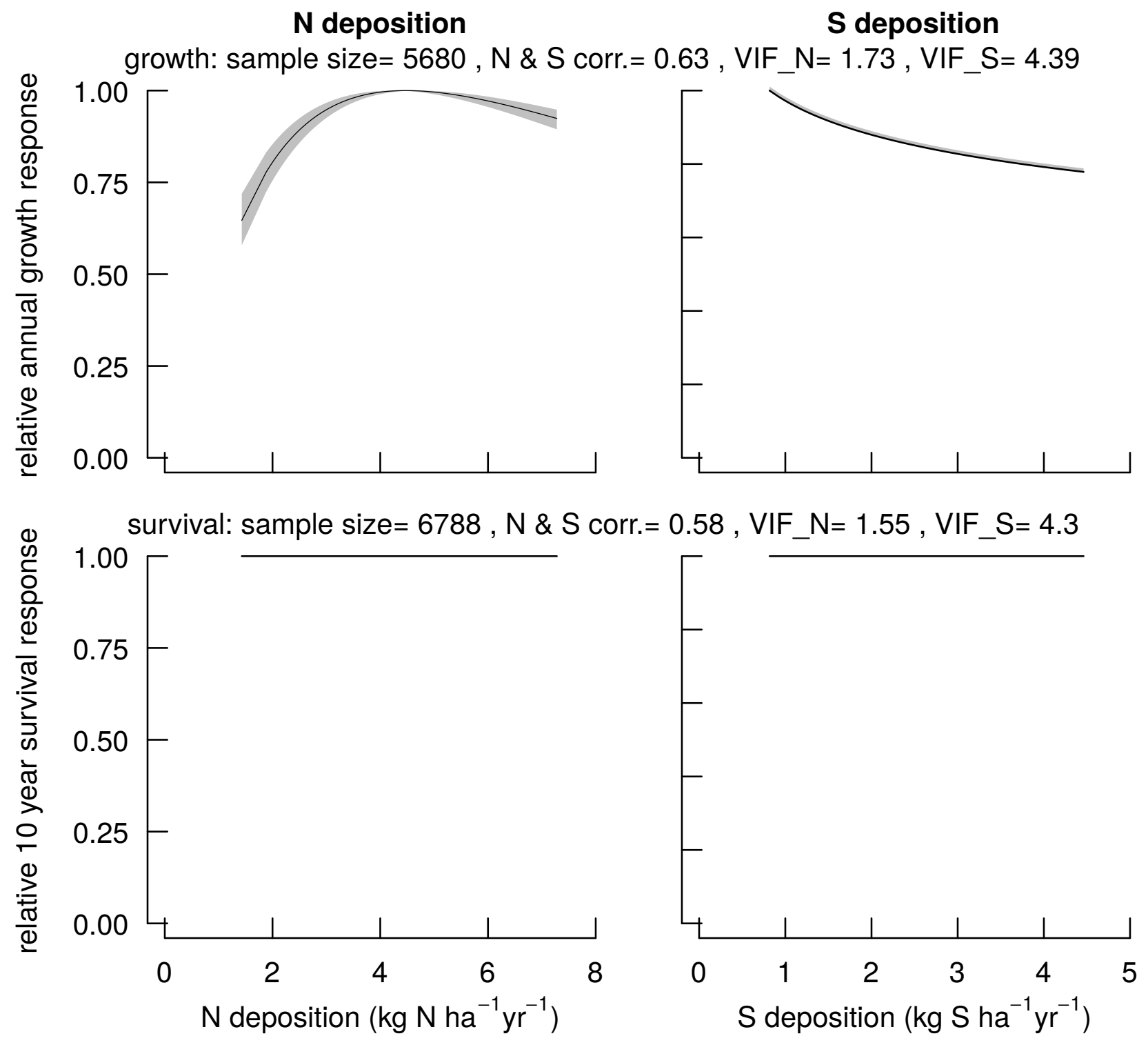




\section{balsam fir}

N deposition

Abies balsamea

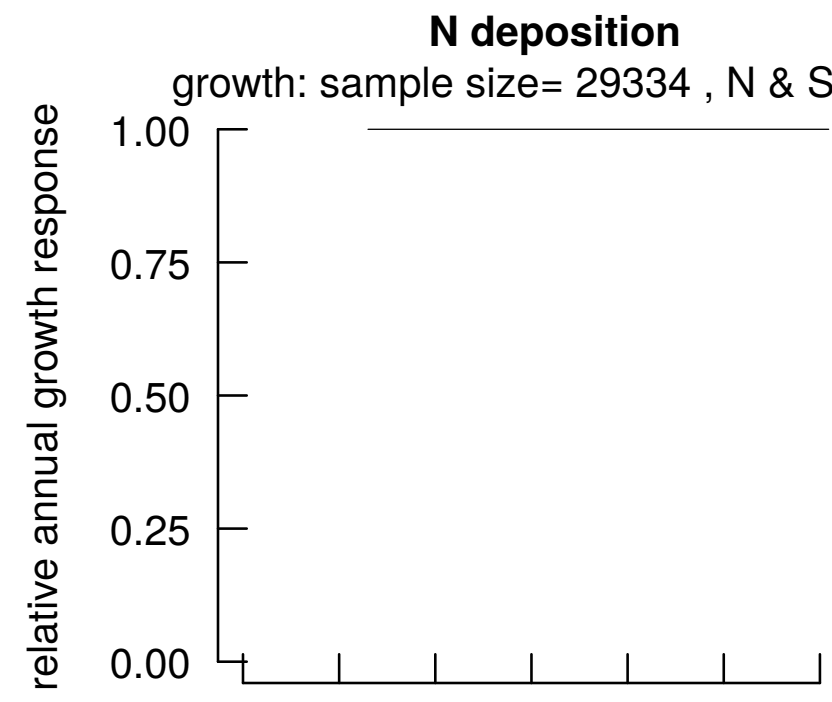

$S$ deposition
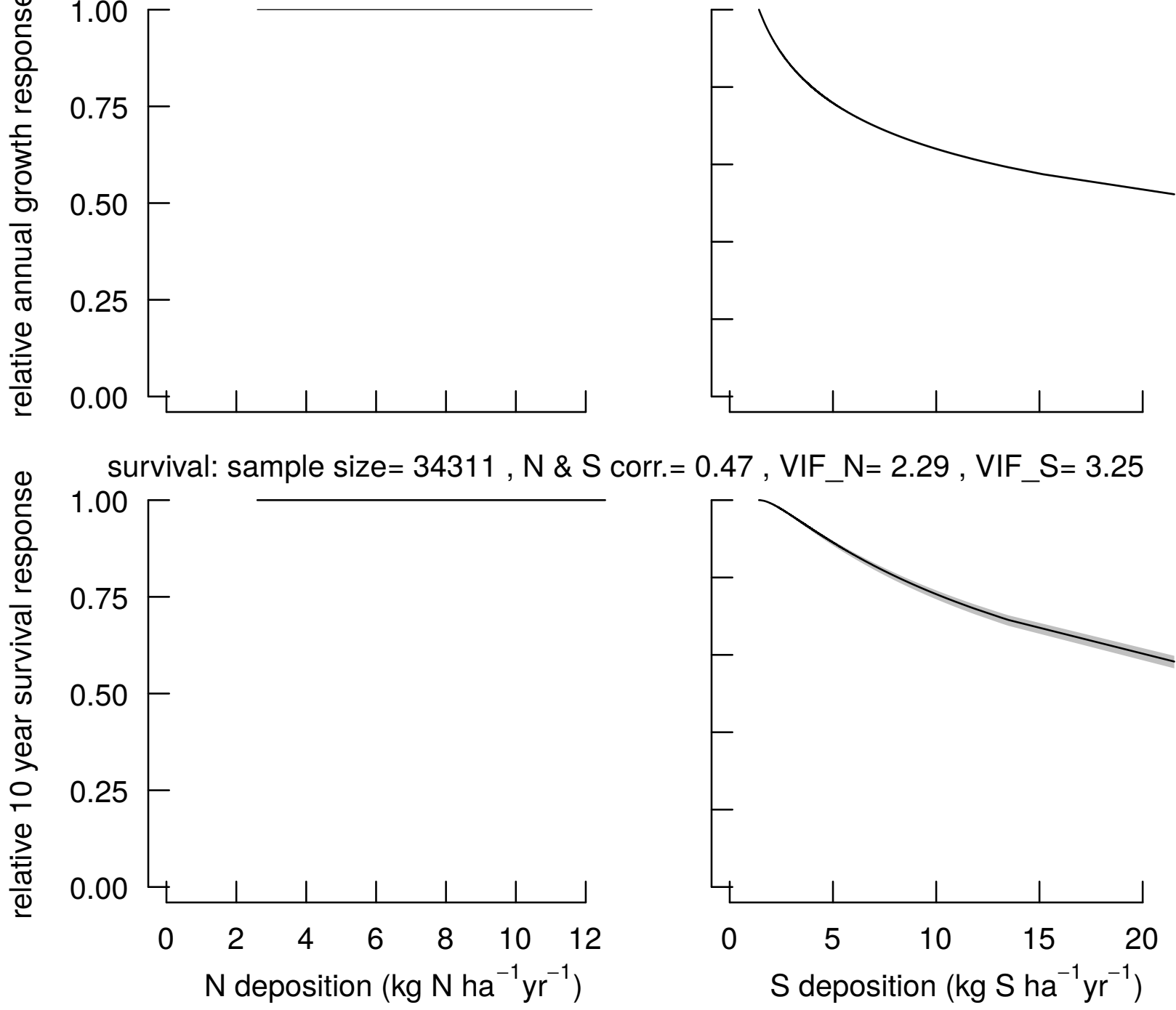


\section{white fir}

Abies concolor

\section{N deposition}
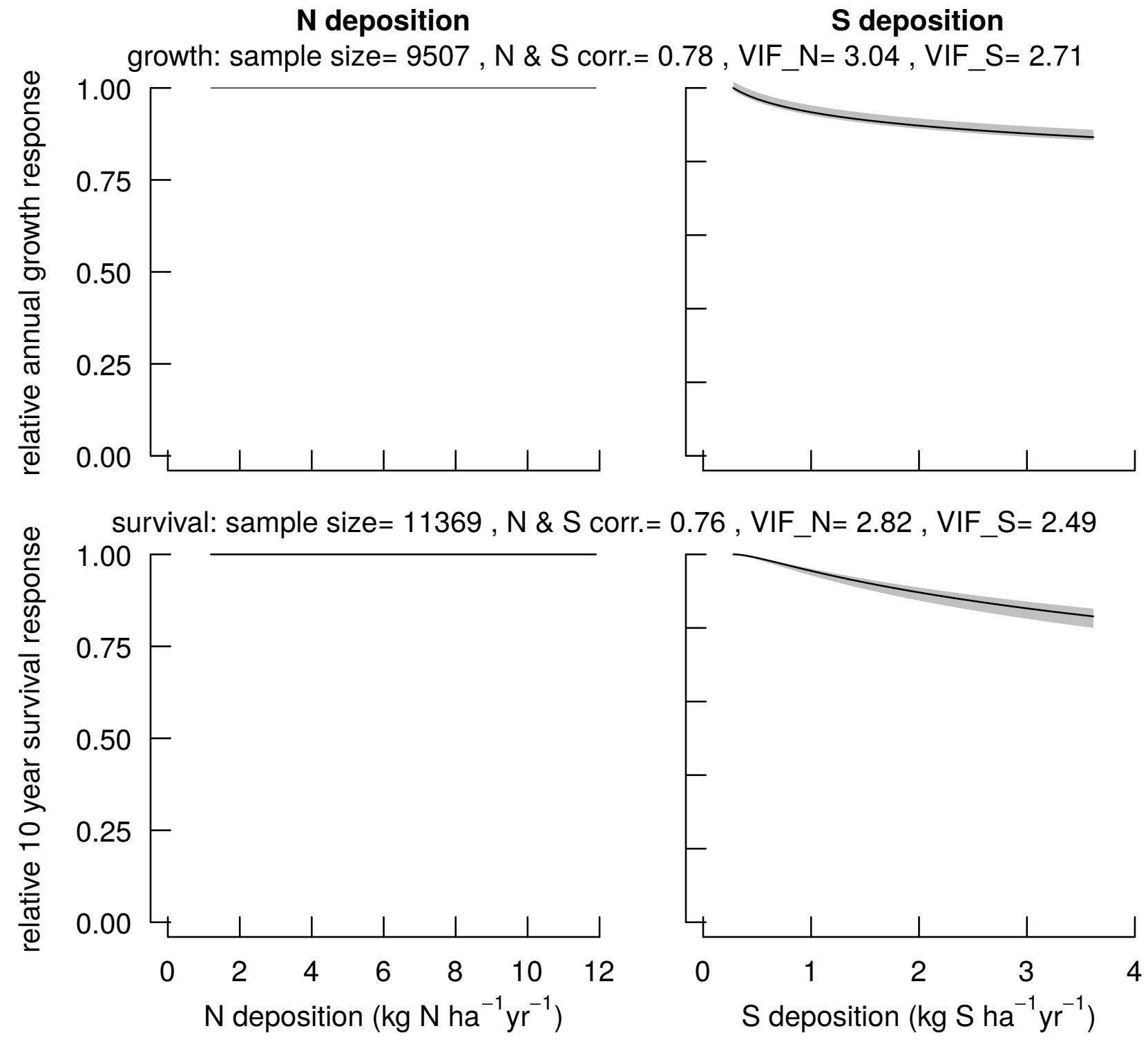


\section{grand fir}

$\mathbf{N}$ deposition

Abies grandis

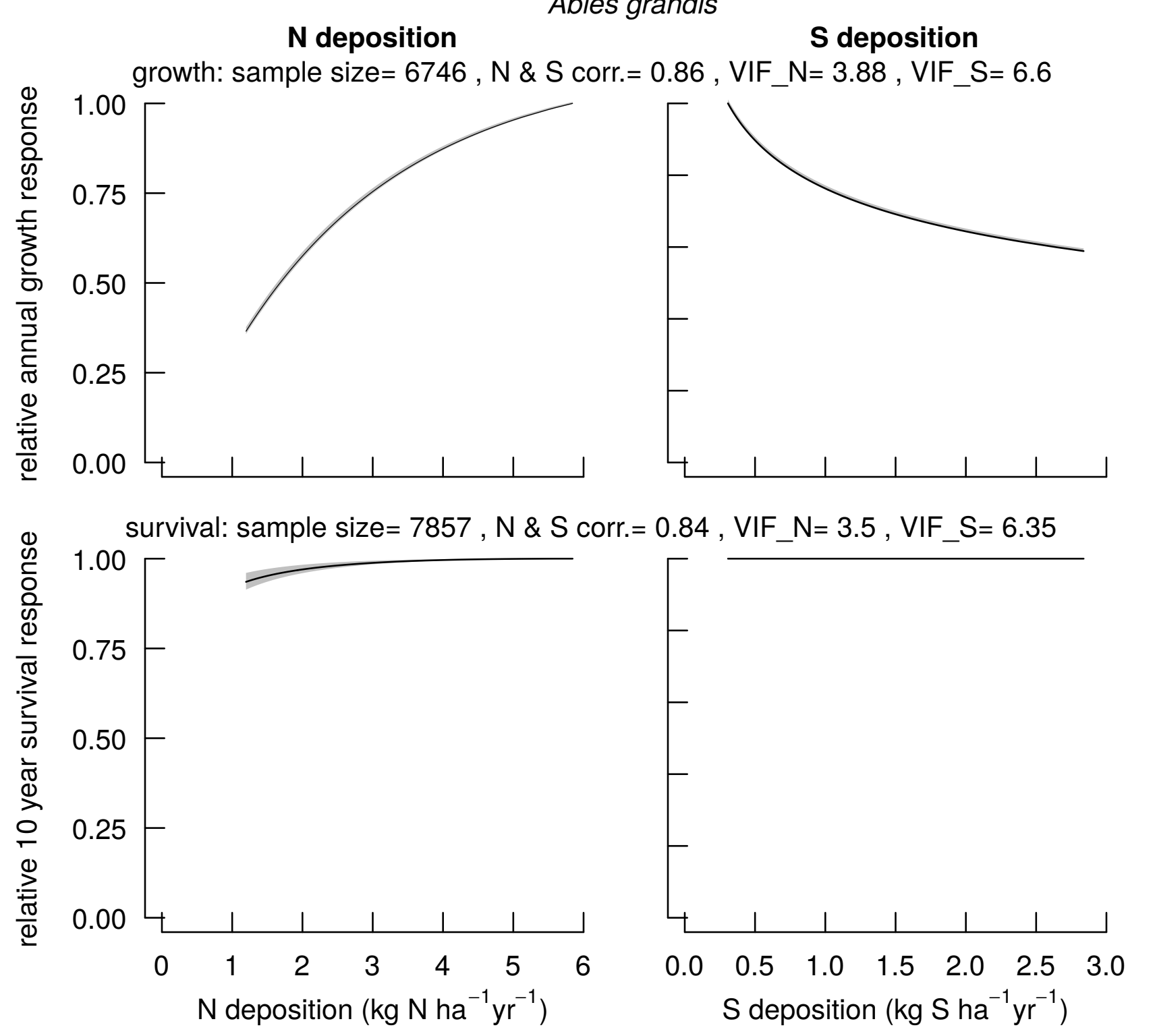




\section{subalpine fir}

N deposition

Abies lasiocarpa

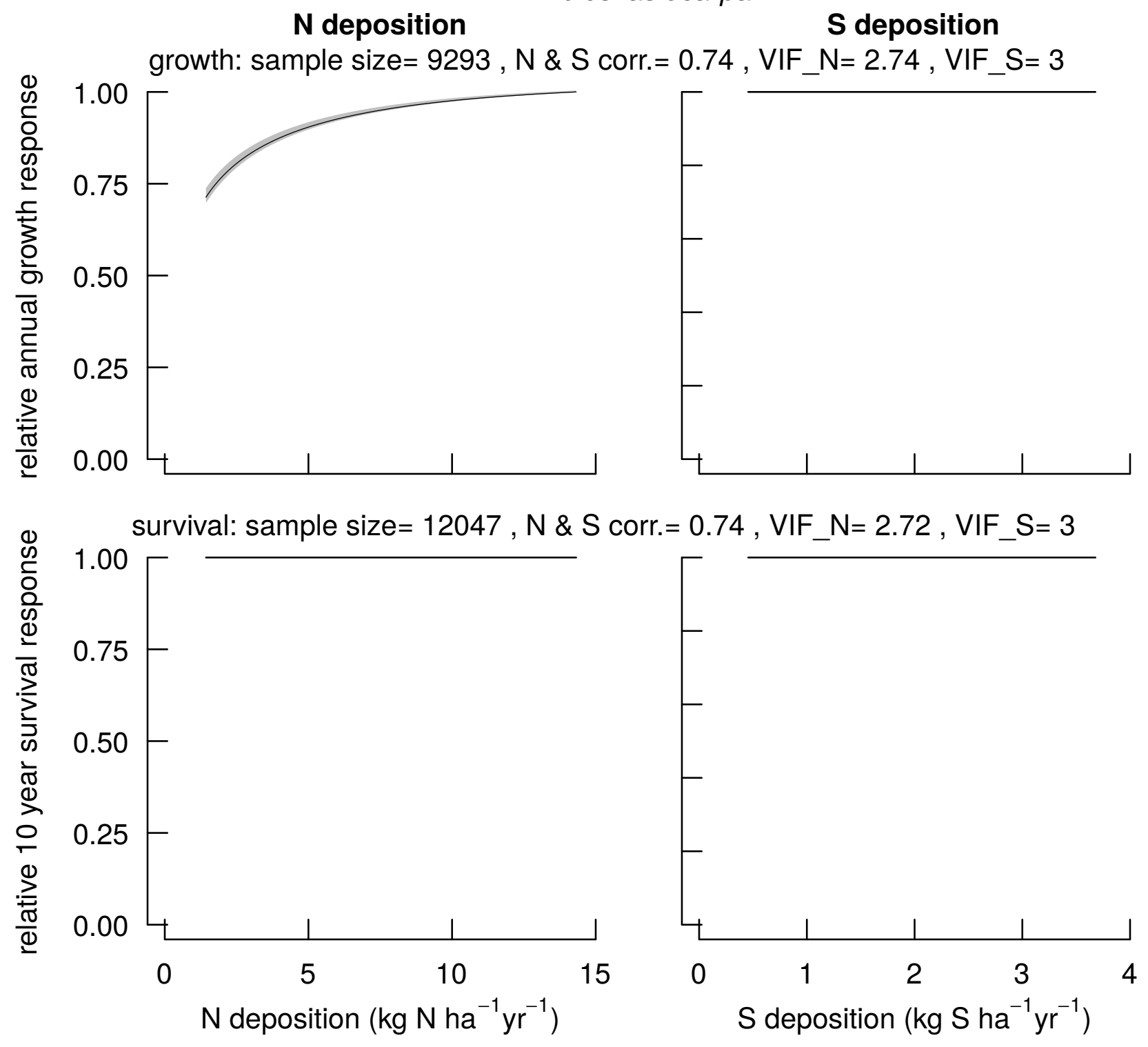




\section{western juniper \\ N deposition \\ Juniperus occidentalis}

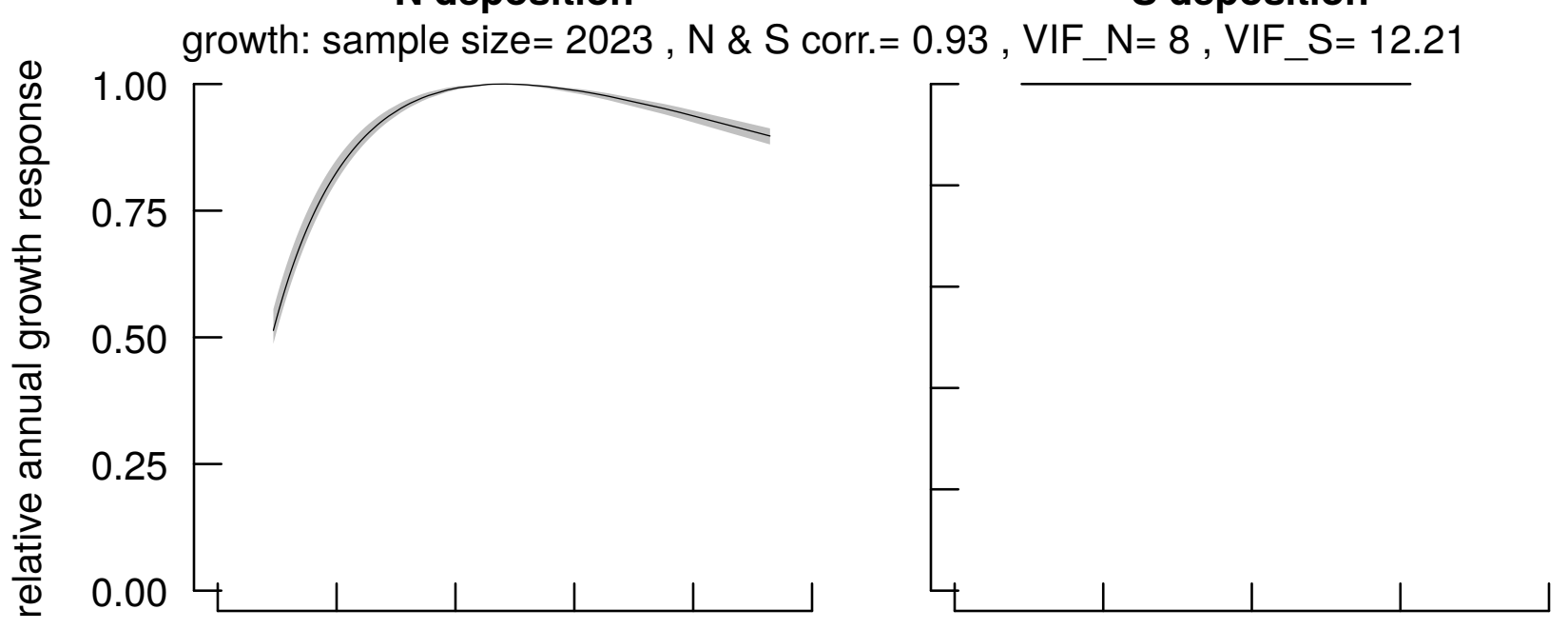

(1) survival: sample size $=2499, \mathrm{~N} \& \mathrm{~S}$ corr. $=0.94, \mathrm{VIF} N \mathrm{~N}=9.25, \mathrm{VIF} \mathrm{S}=14.89$
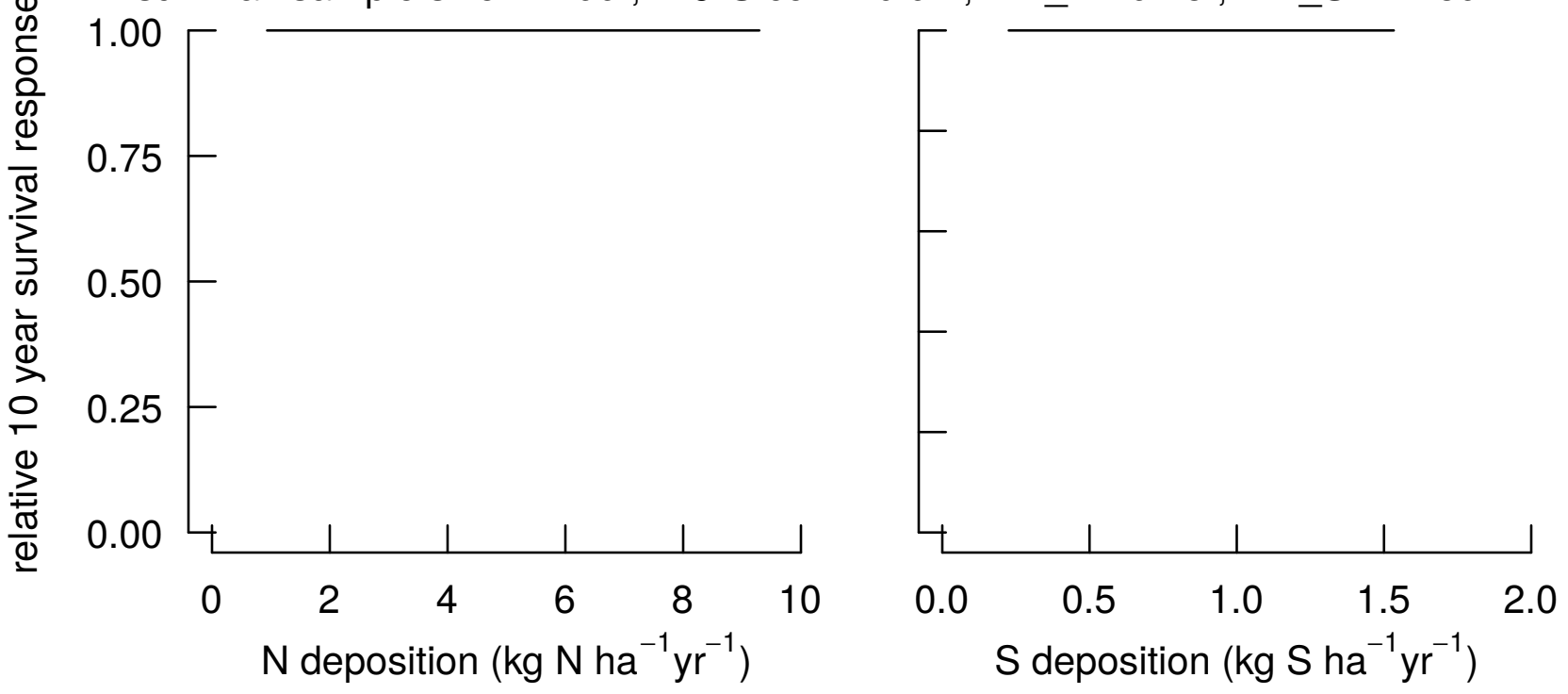


\section{Utah juniper \\ N deposition \\ Juniperus osteosperma \\ S deposition}
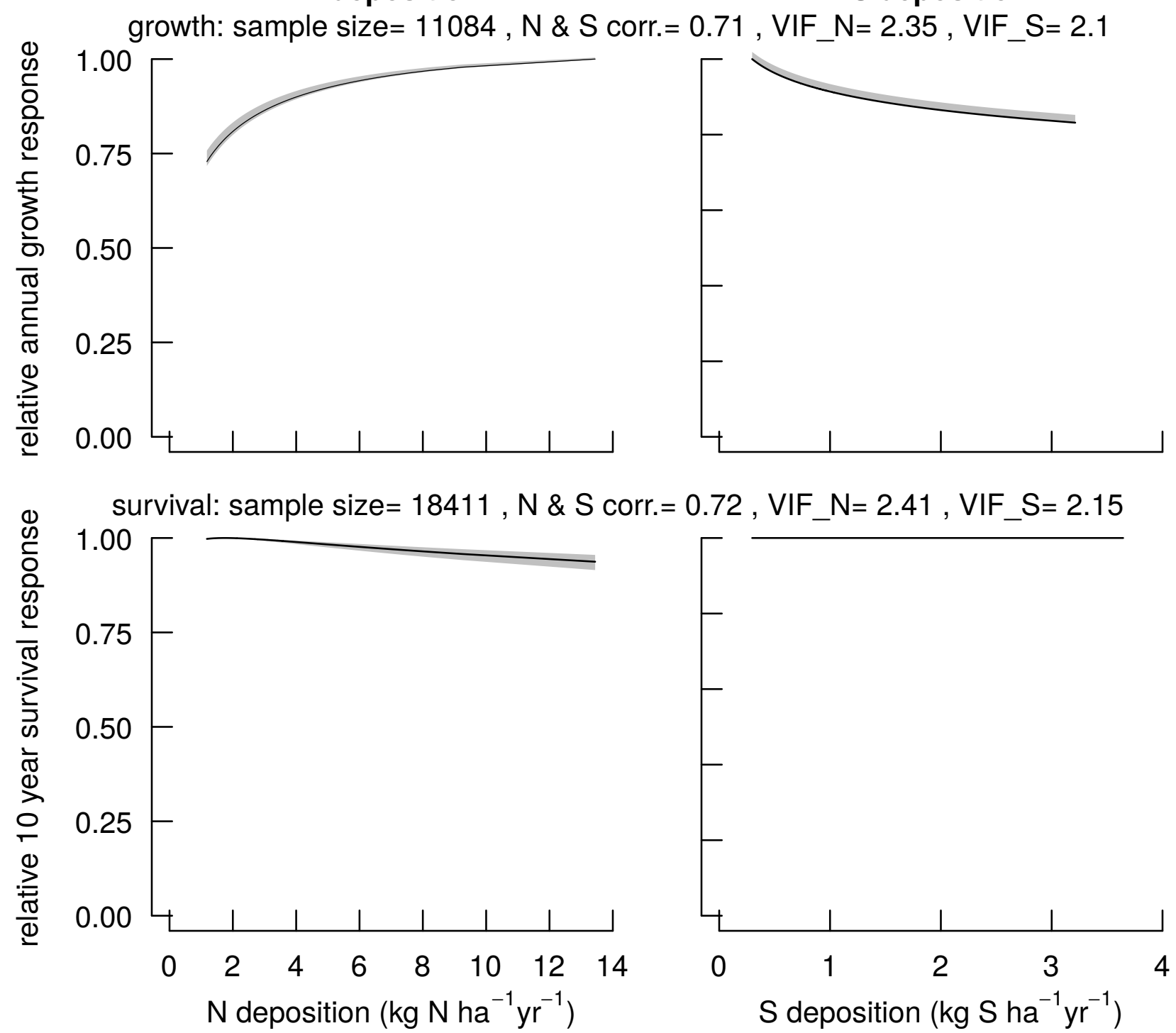


\section{eastern redcedar}

N deposition

Juniperus virginiana

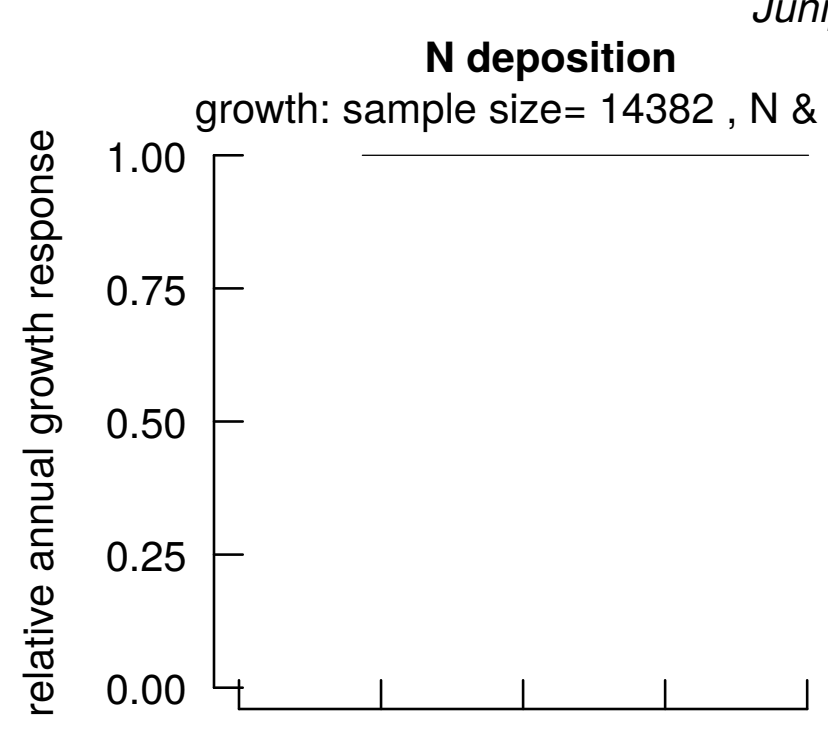

$S$ deposition
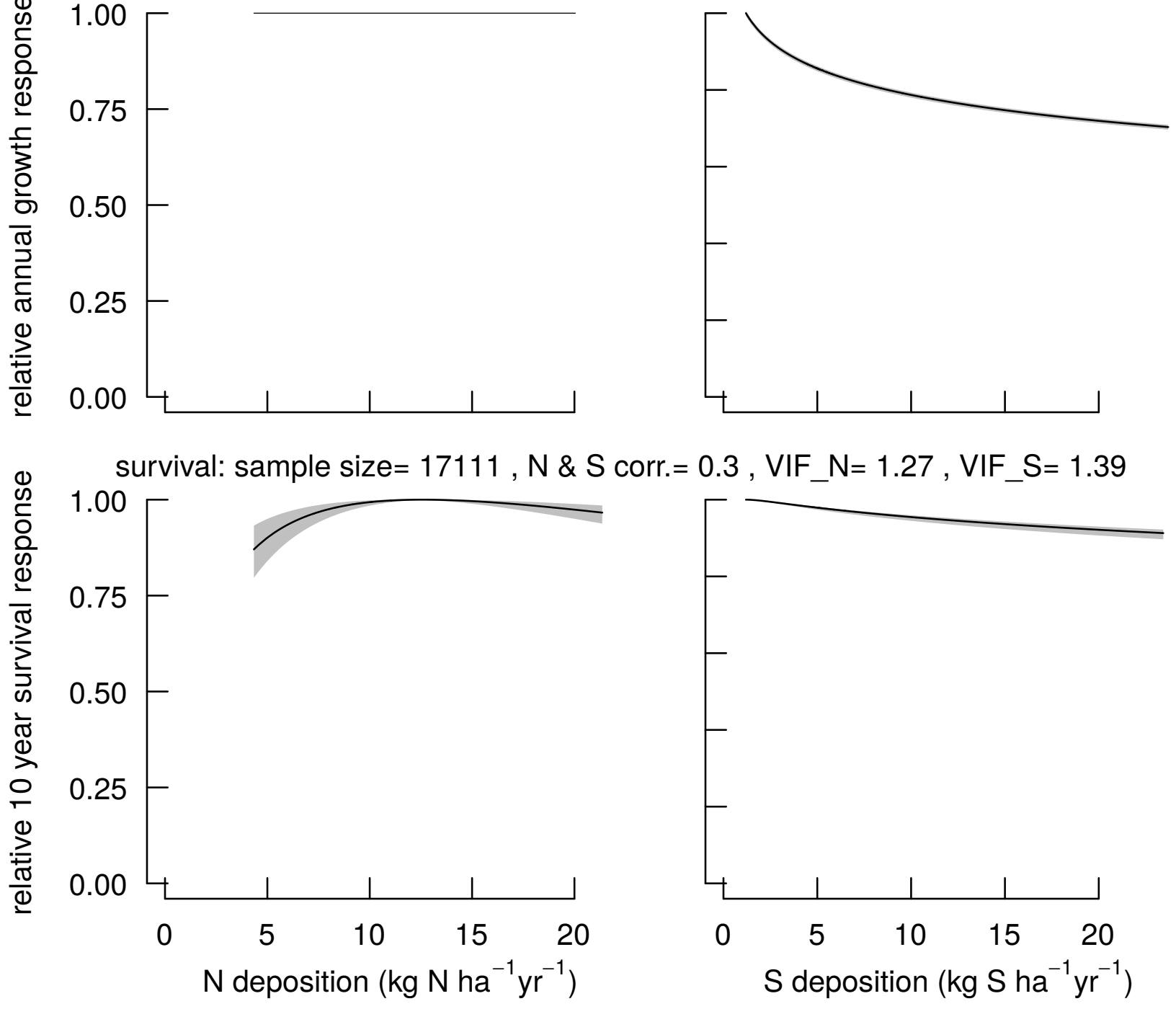

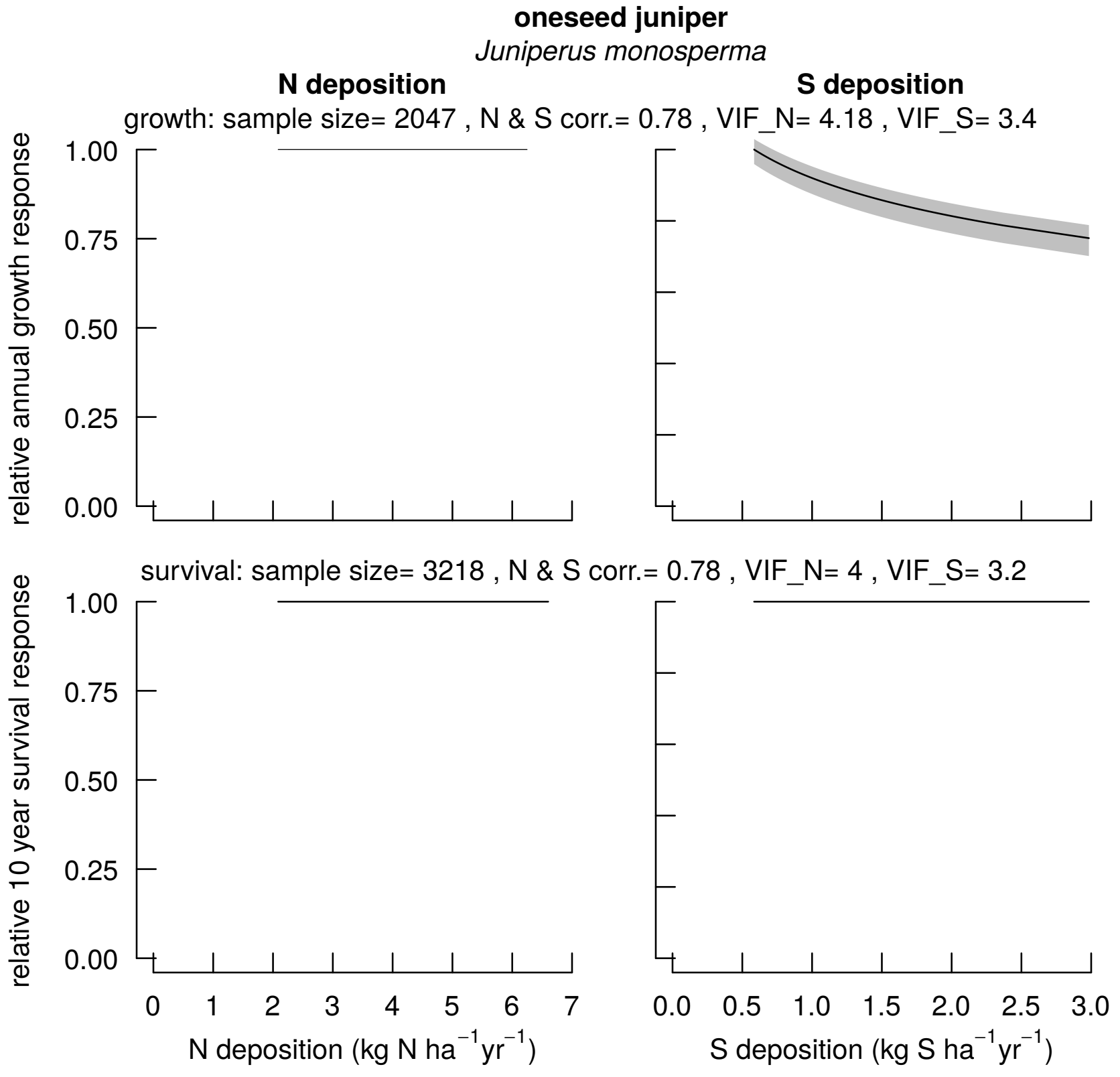


\section{tamarack (native)}

Larix laricina

\section{N deposition}
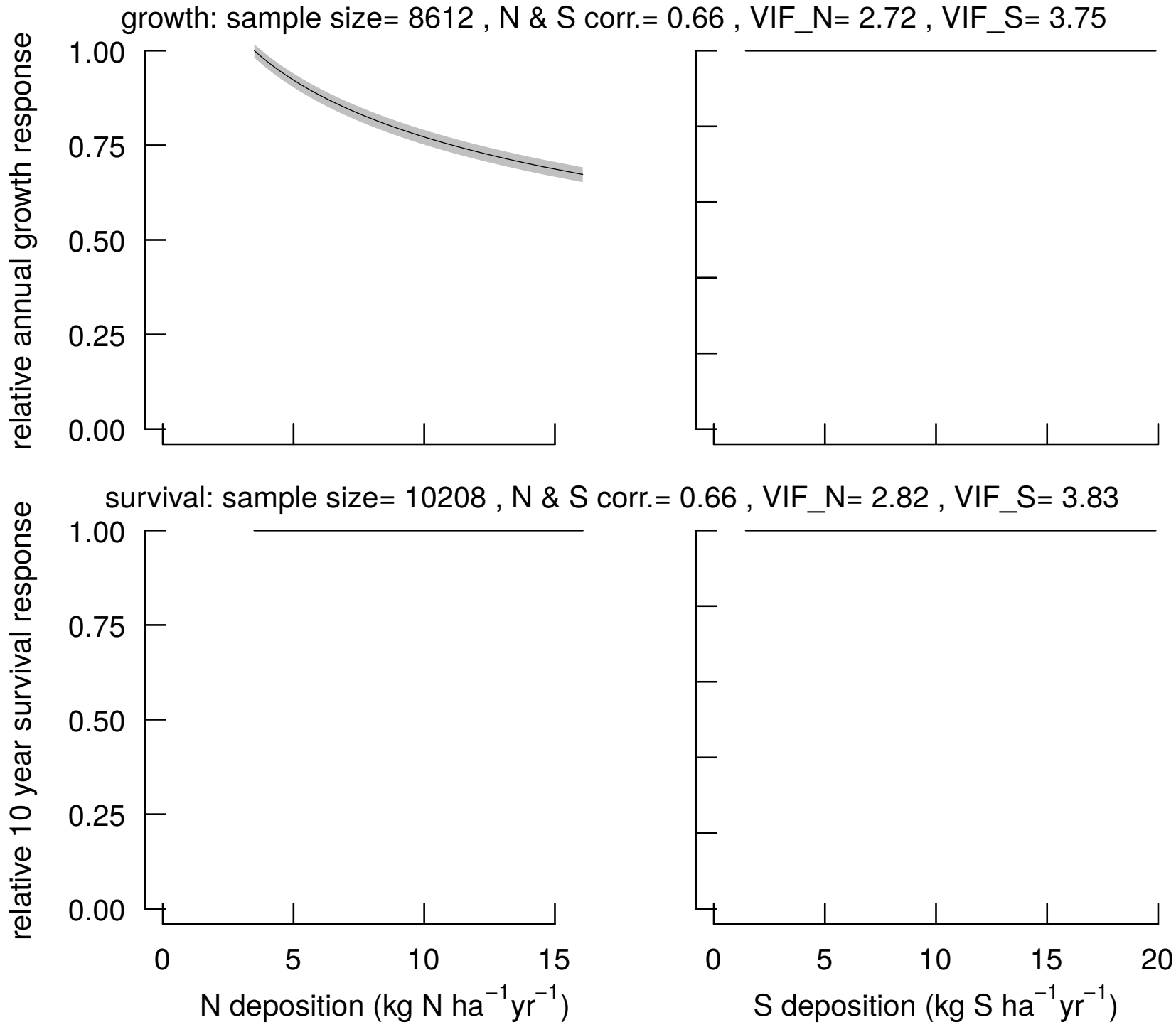


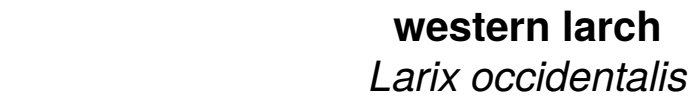

$\mathbf{N}$ deposition

Larix occidentalis

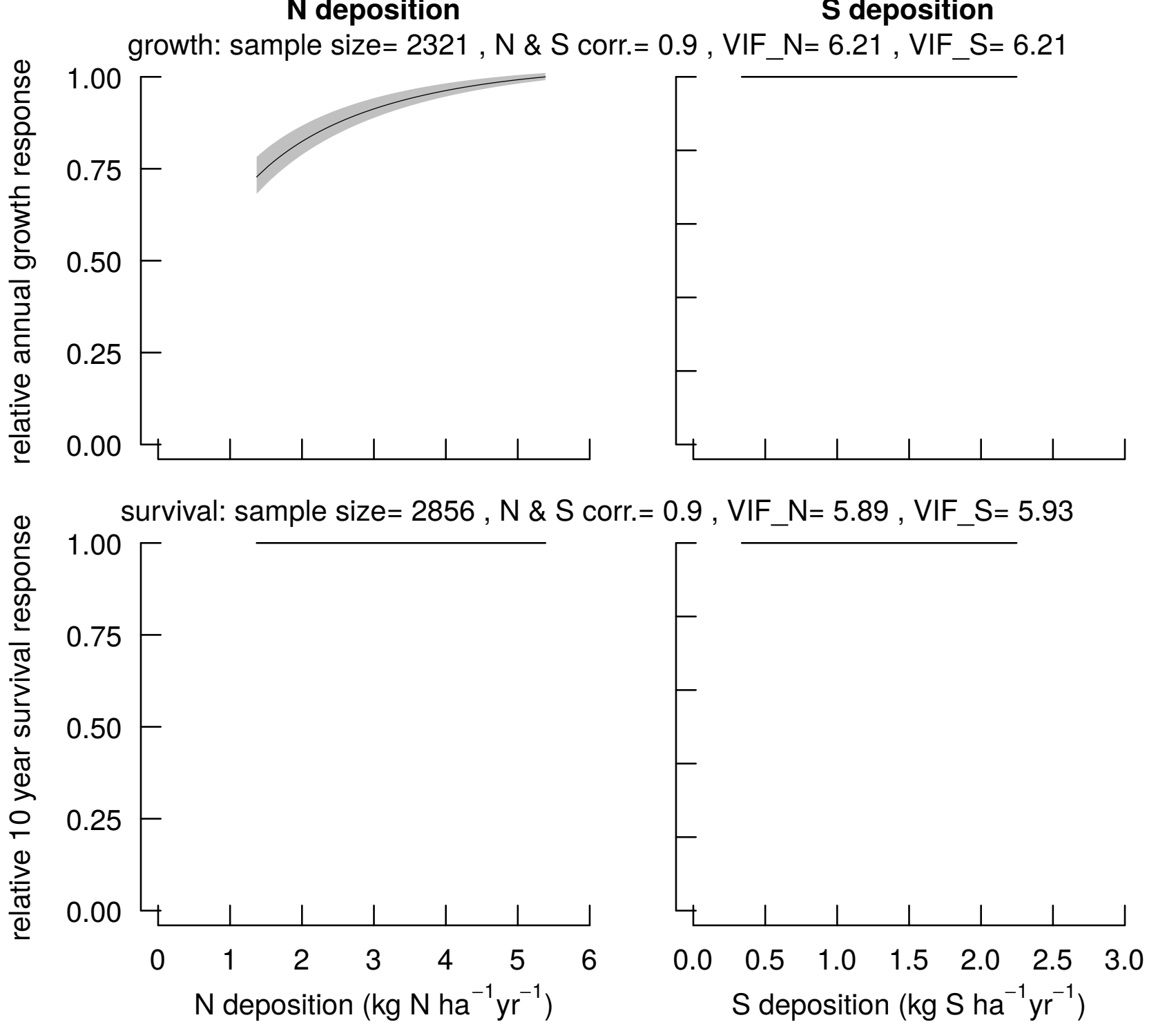




\section{incense-cedar}

Calocedrus decurrens

\section{$\mathbf{N}$ deposition}

S deposition

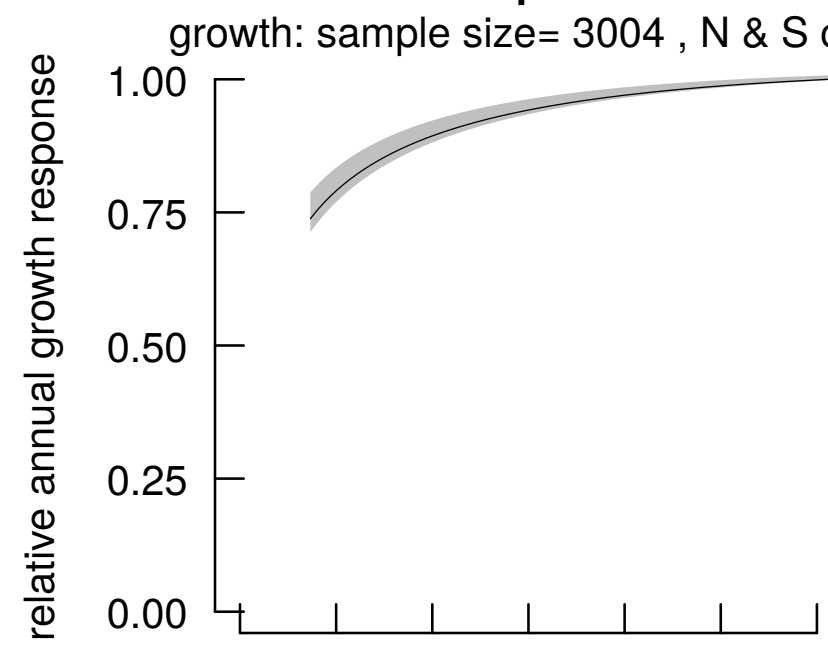

0.83, VIF_N $=7.49$, VIF_S $=10.29$
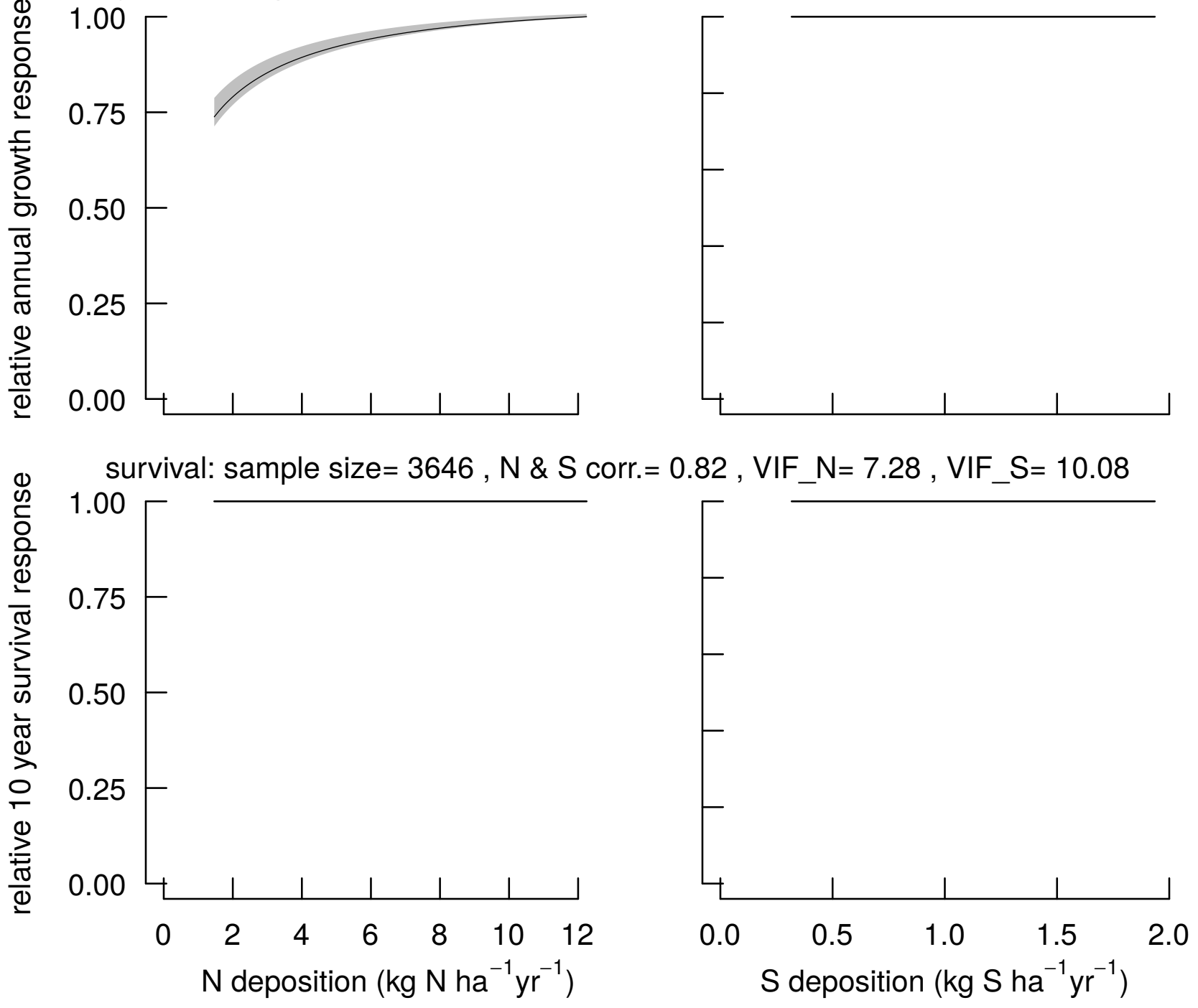


\section{Engelmann spruce}

$\mathbf{N}$ deposition

Picea engelmannii

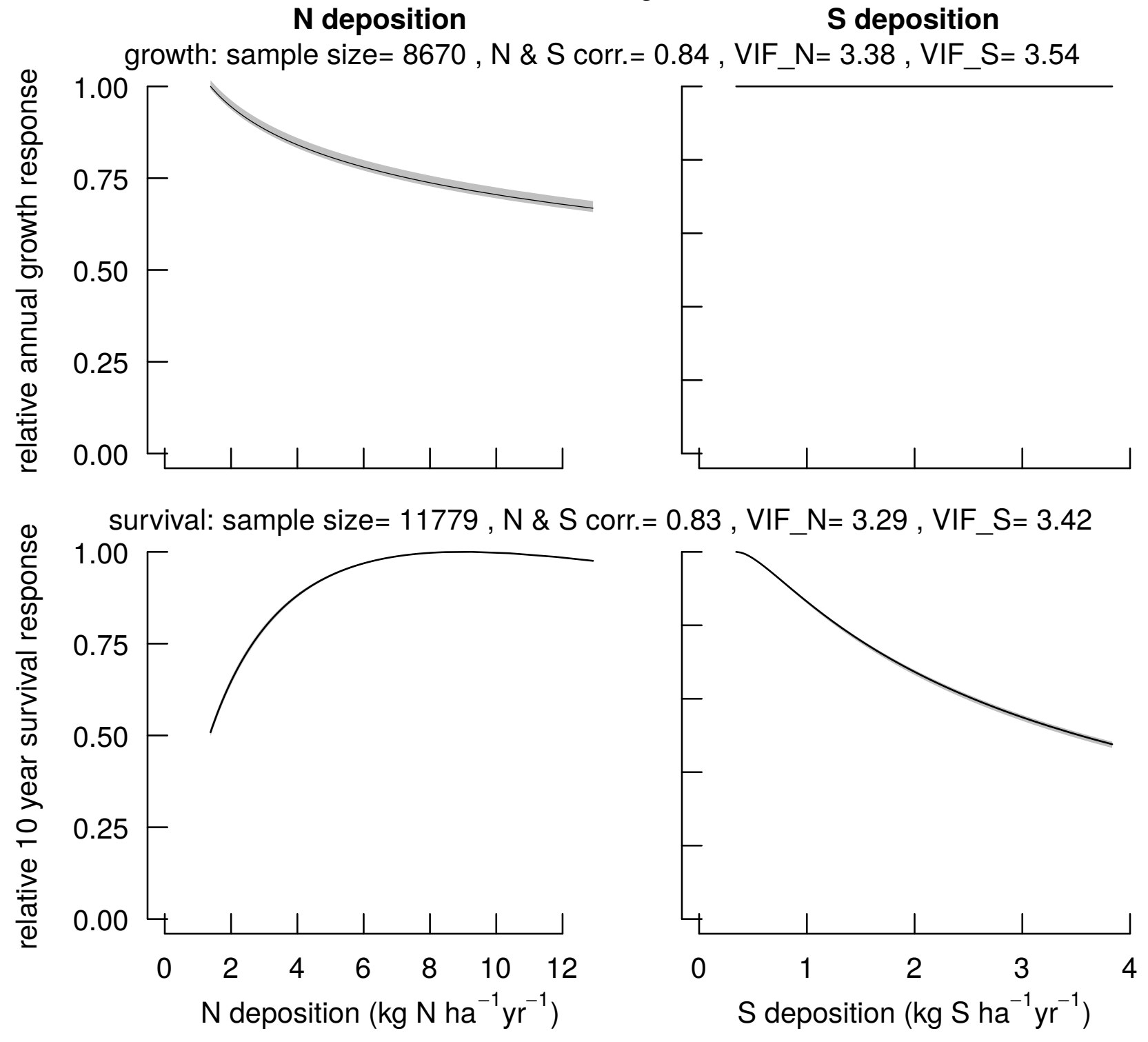




\section{white spruce}

$\mathbf{N}$ deposition

Picea glauca

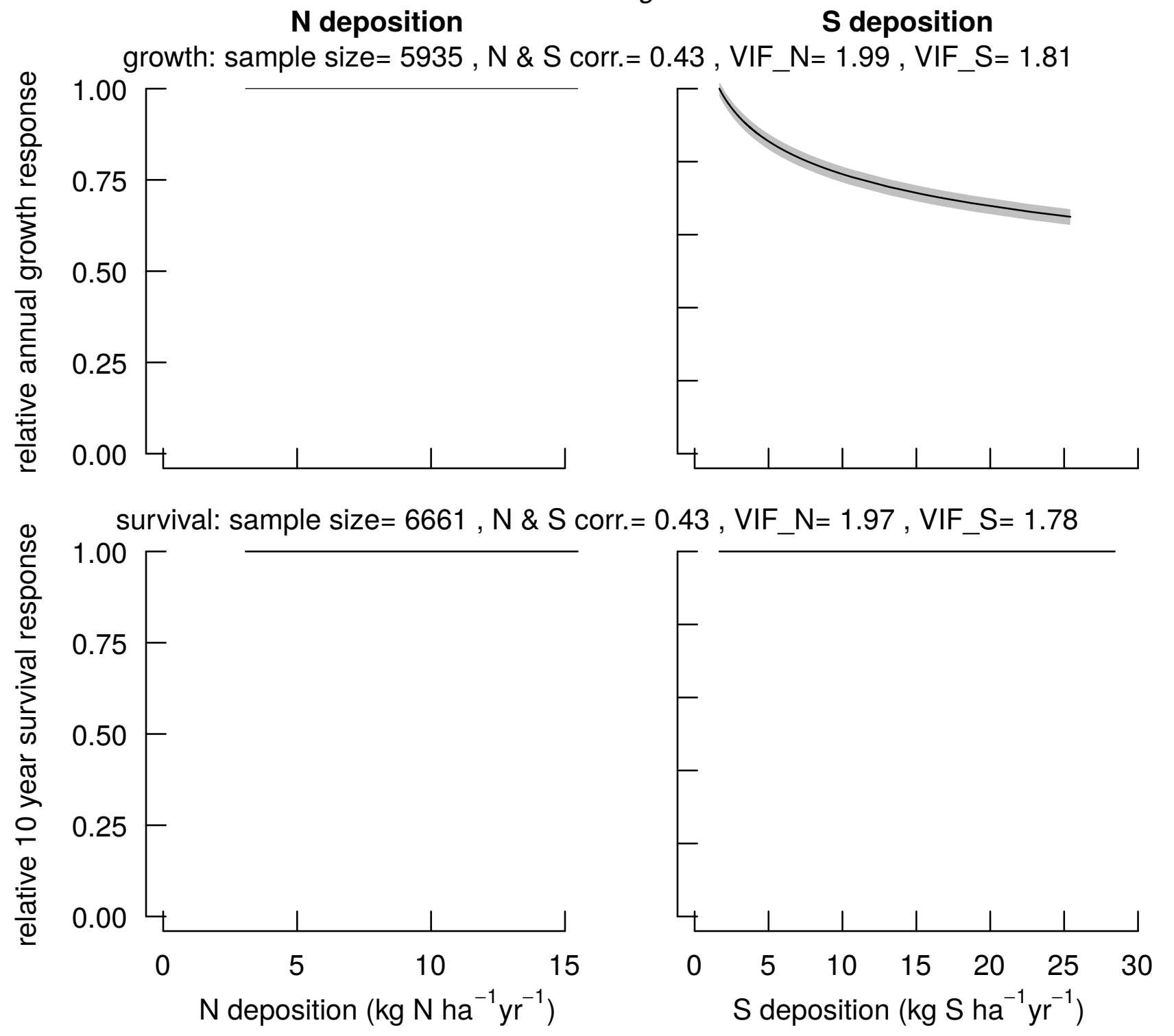




\section{black spruce}

N deposition

Picea mariana
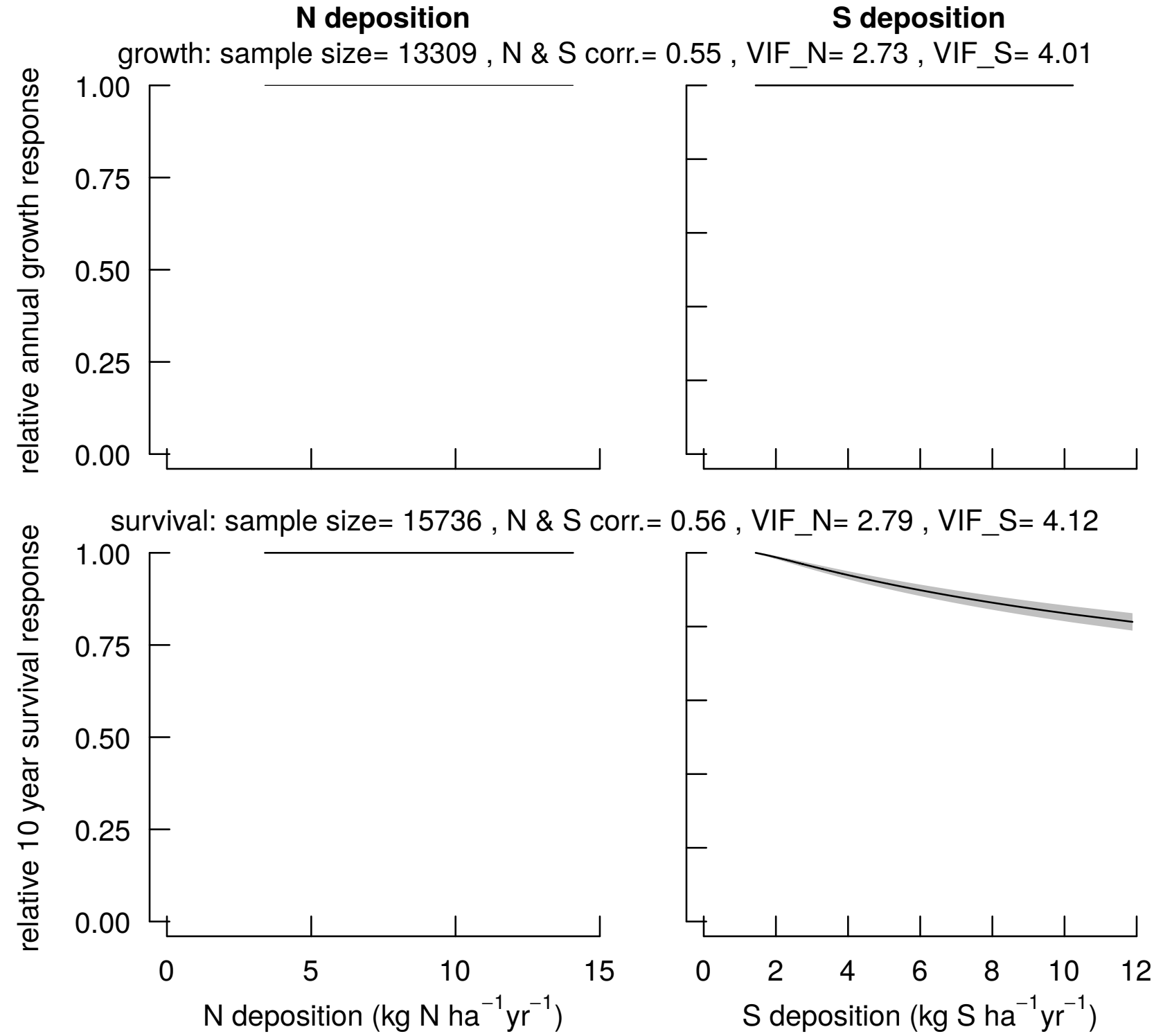


\section{red spruce}

$\mathbf{N}$ deposition

Picea rubens

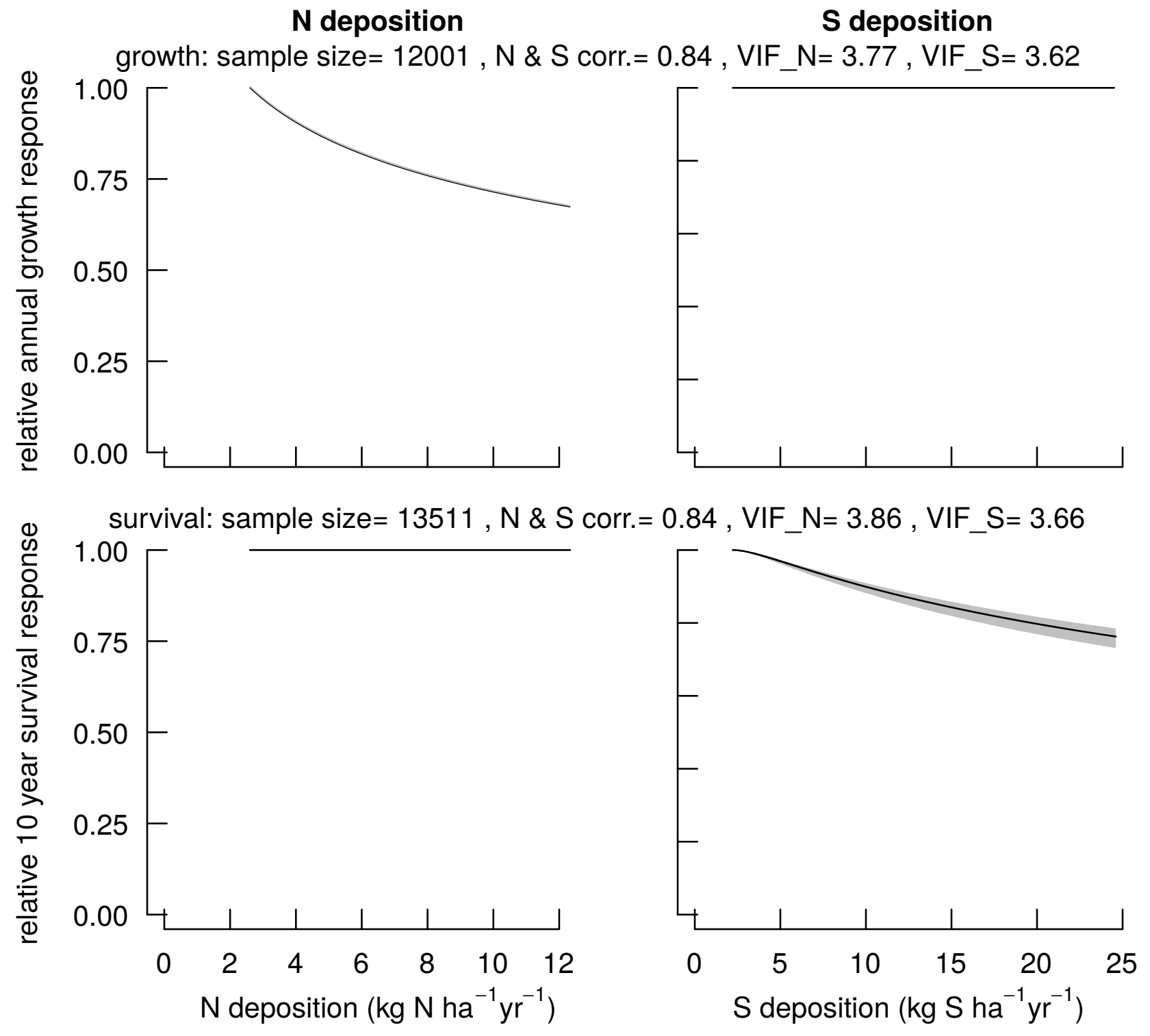



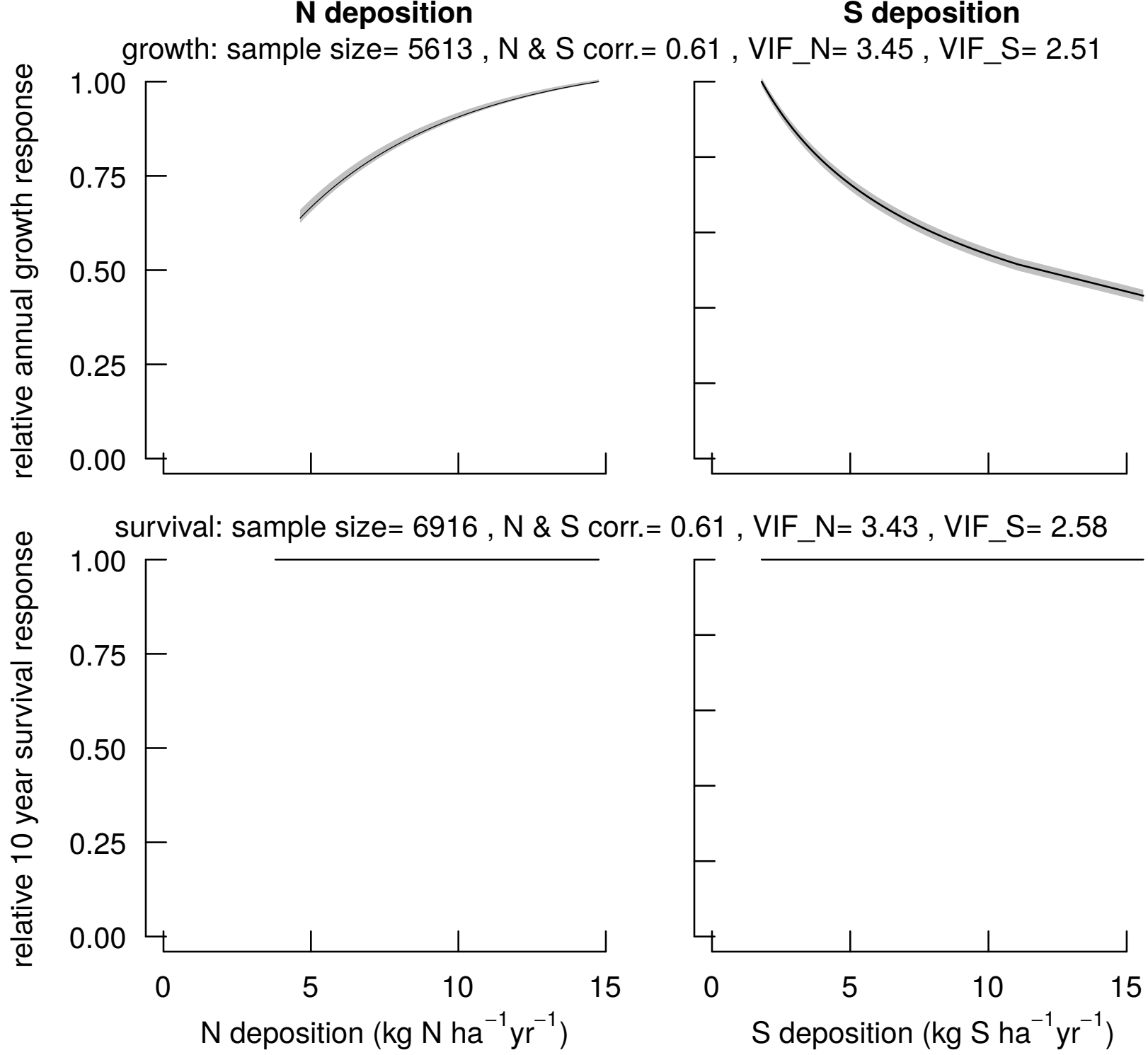


\section{common or two-needle pinyon \\ Pinus edulis}

N deposition

$\mathrm{S}$ deposition
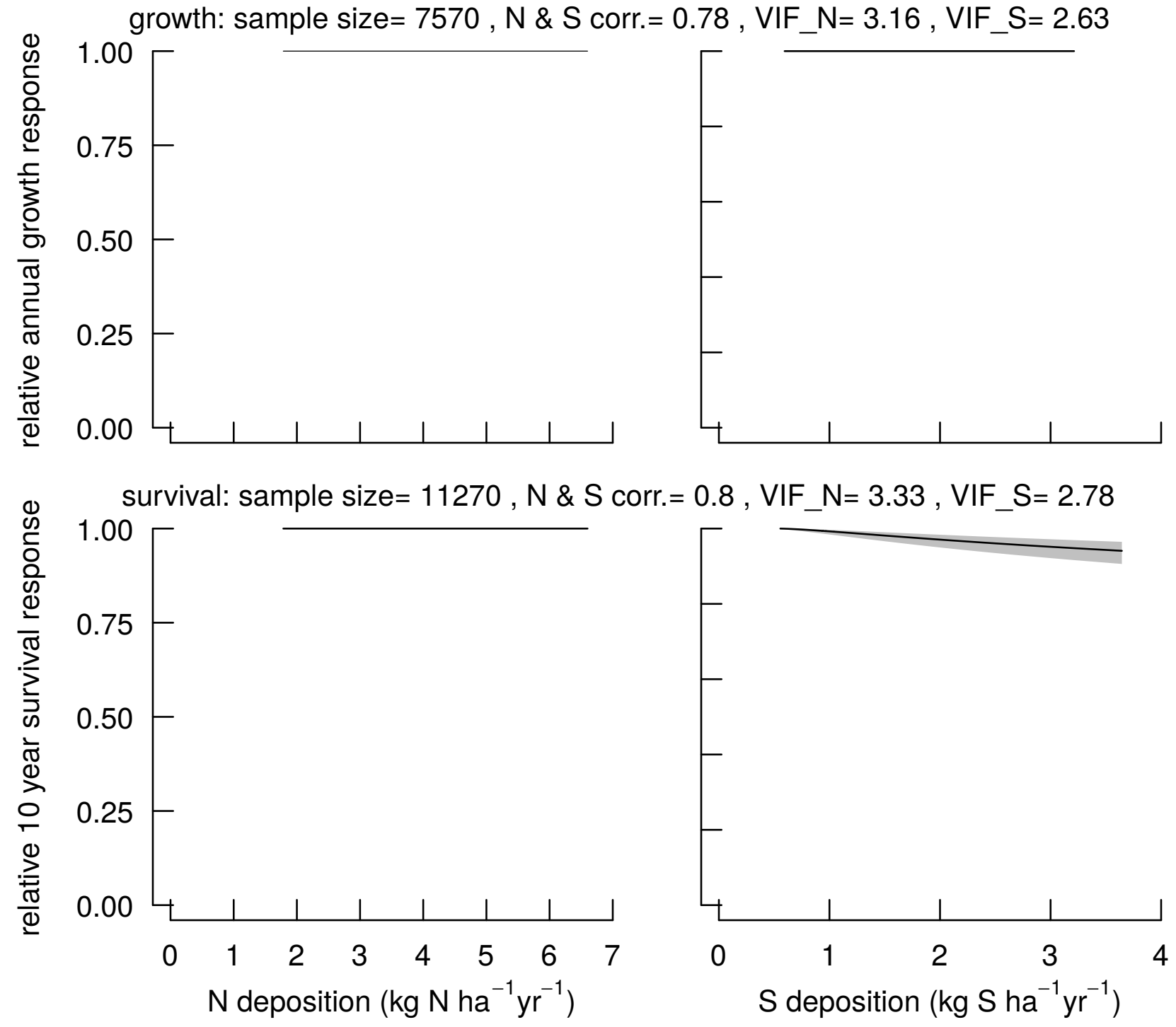


\title{
lodgepole pine
}

Pinus contorta

\section{$\mathrm{N}$ deposition}

\author{
$\mathrm{S}$ deposition
}
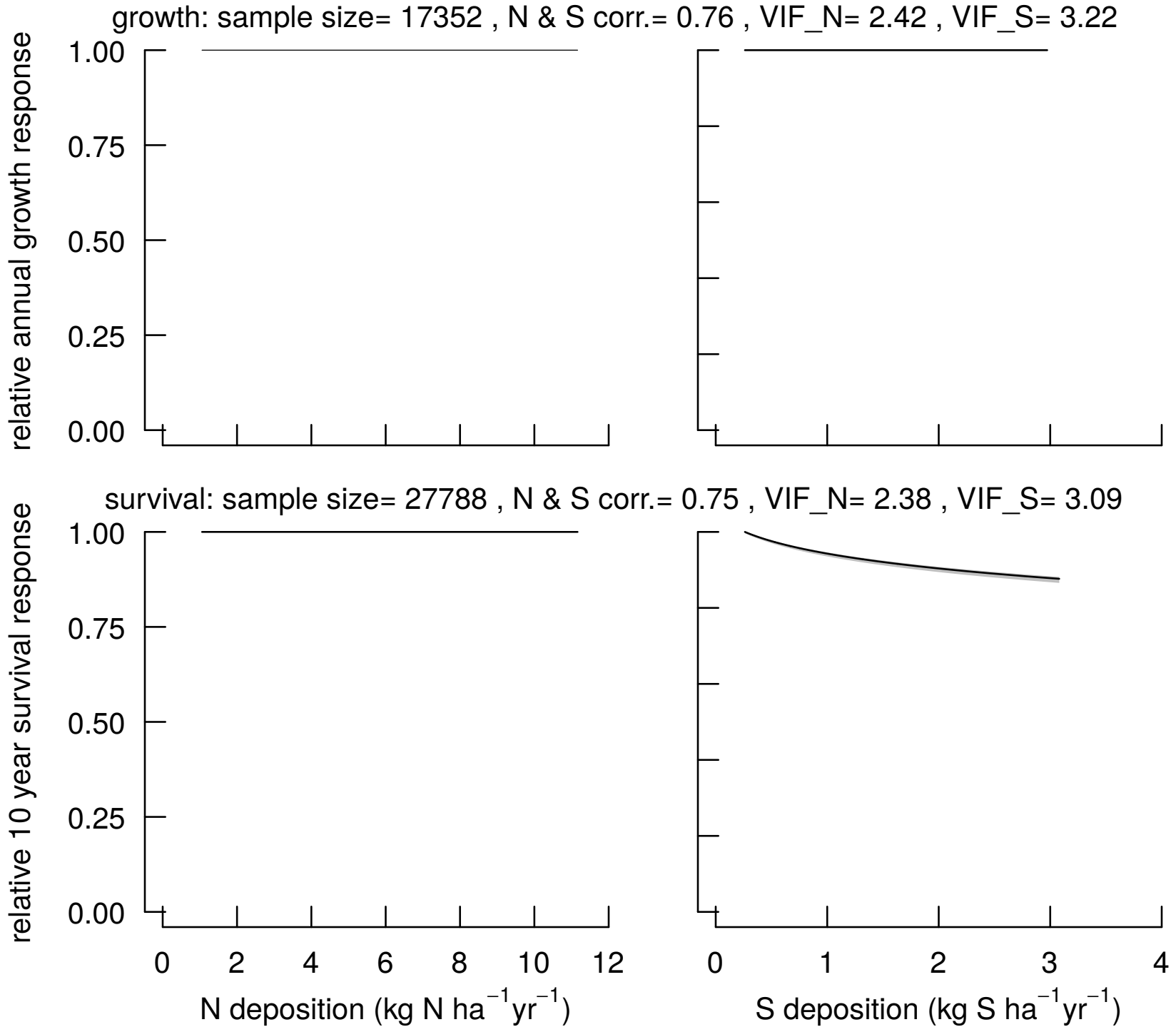


\section{shortleaf pine}

Pinus echinata

\section{$\mathbf{N}$ deposition}

$S$ deposition
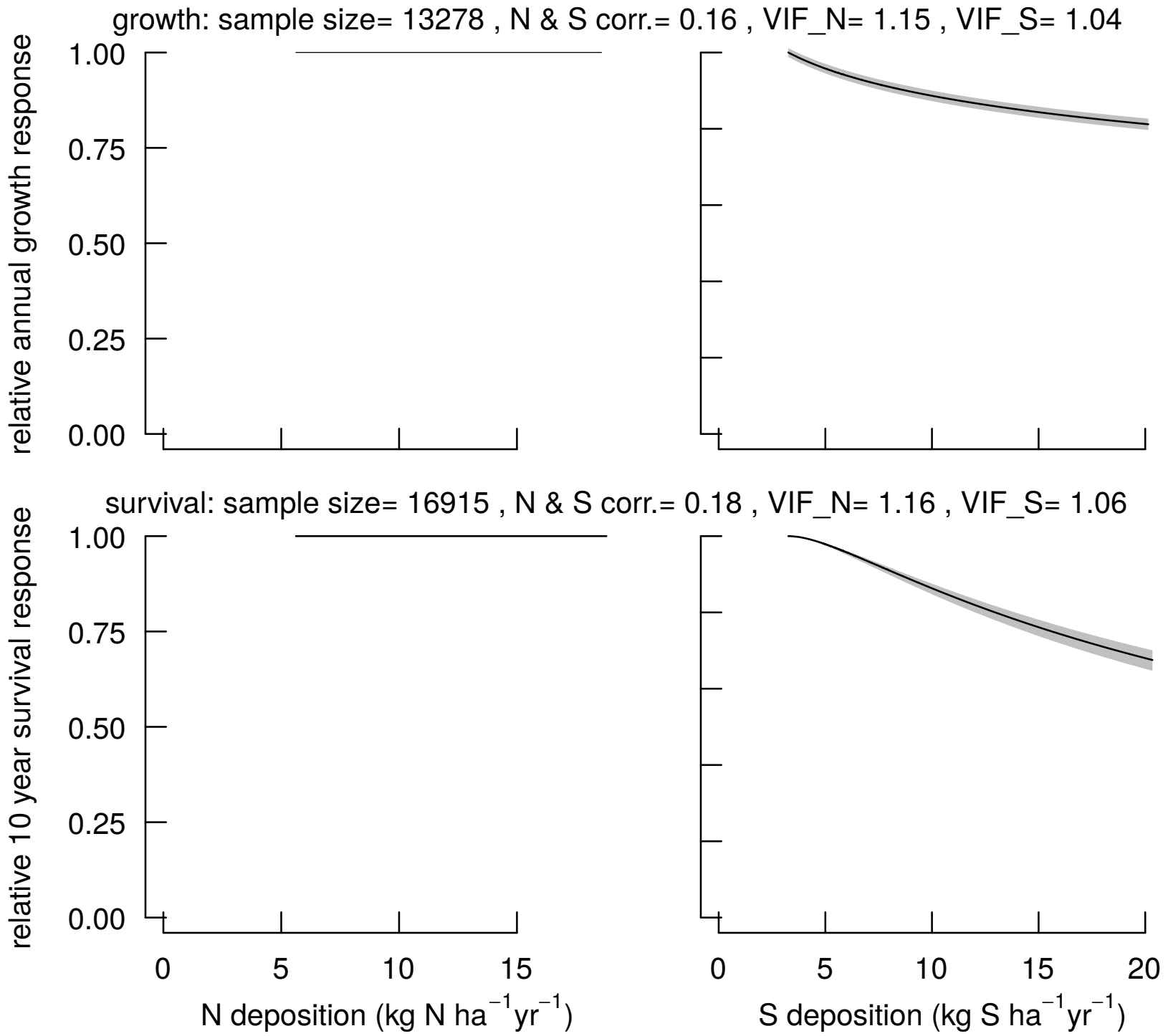
slash pine

N deposition

Pinus elliottii

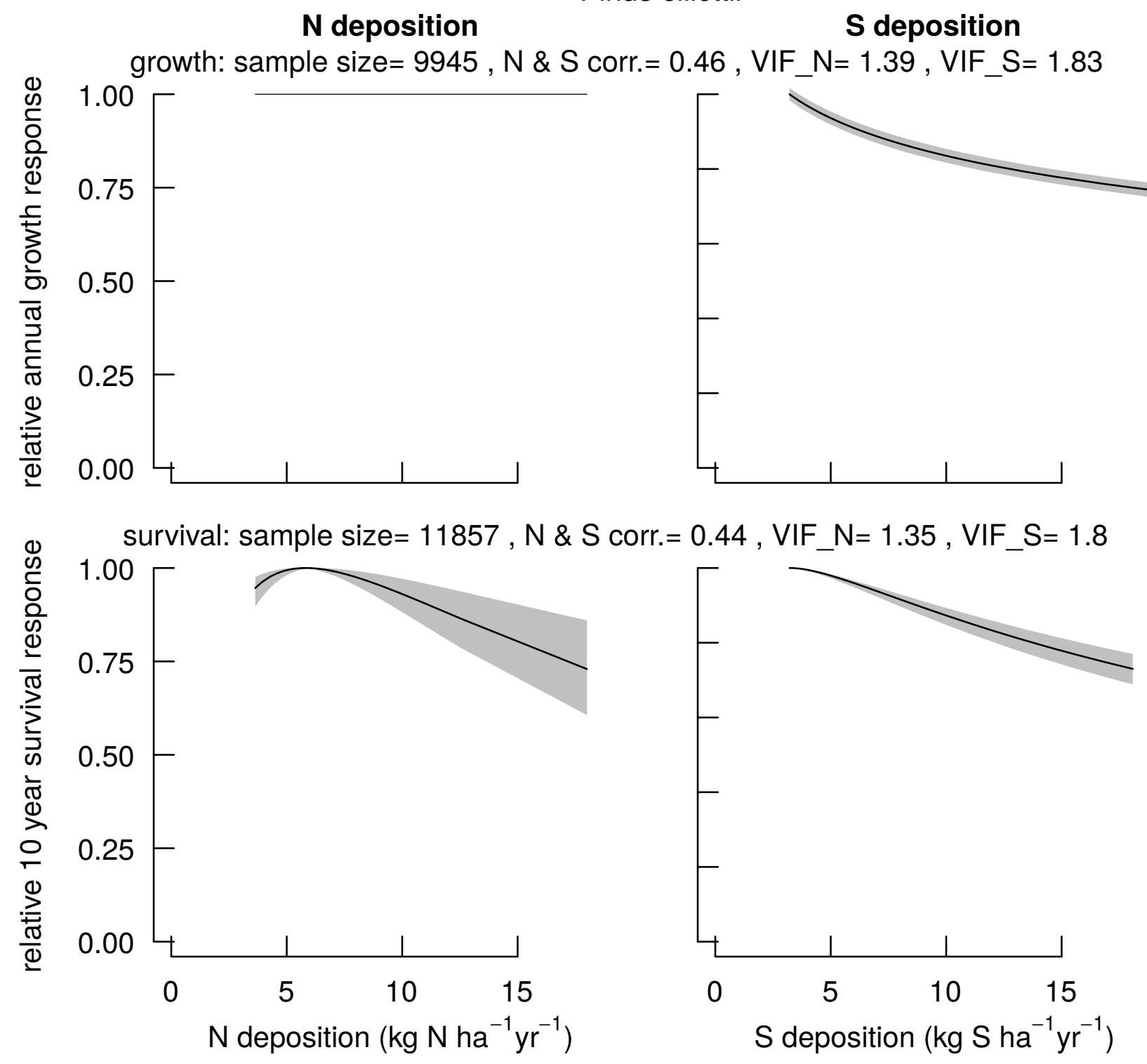




\section{longleaf pine}

$\mathbf{N}$ deposition

Pinus palustris

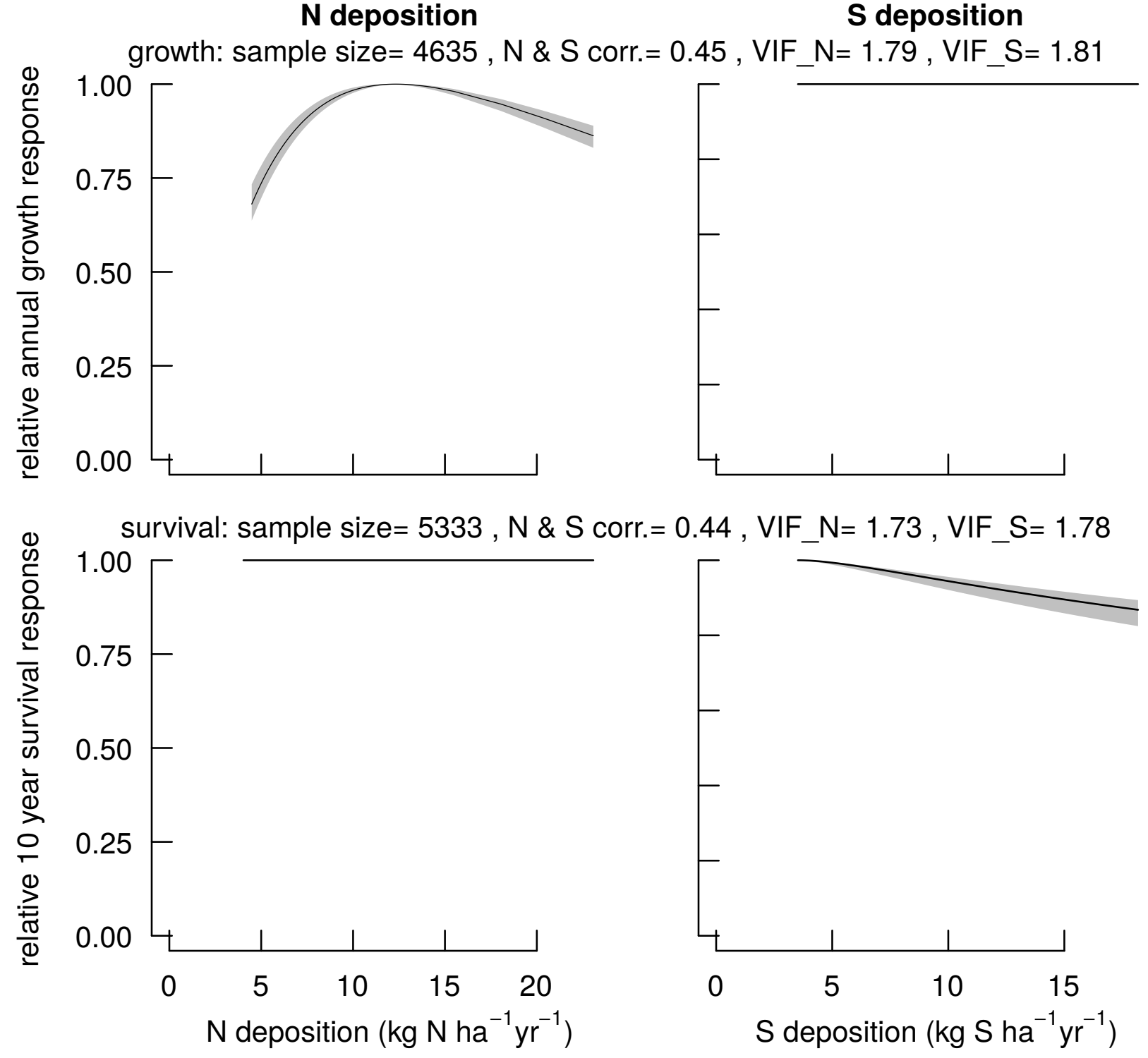


$\mathrm{N}$ deposition

ponderosa pine

Pinus ponderosa

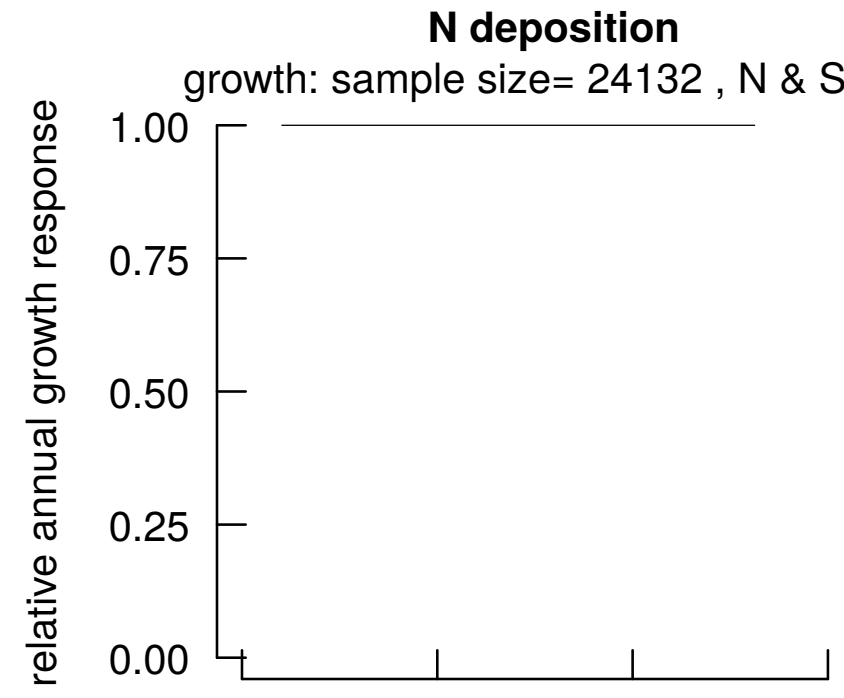

S deposition
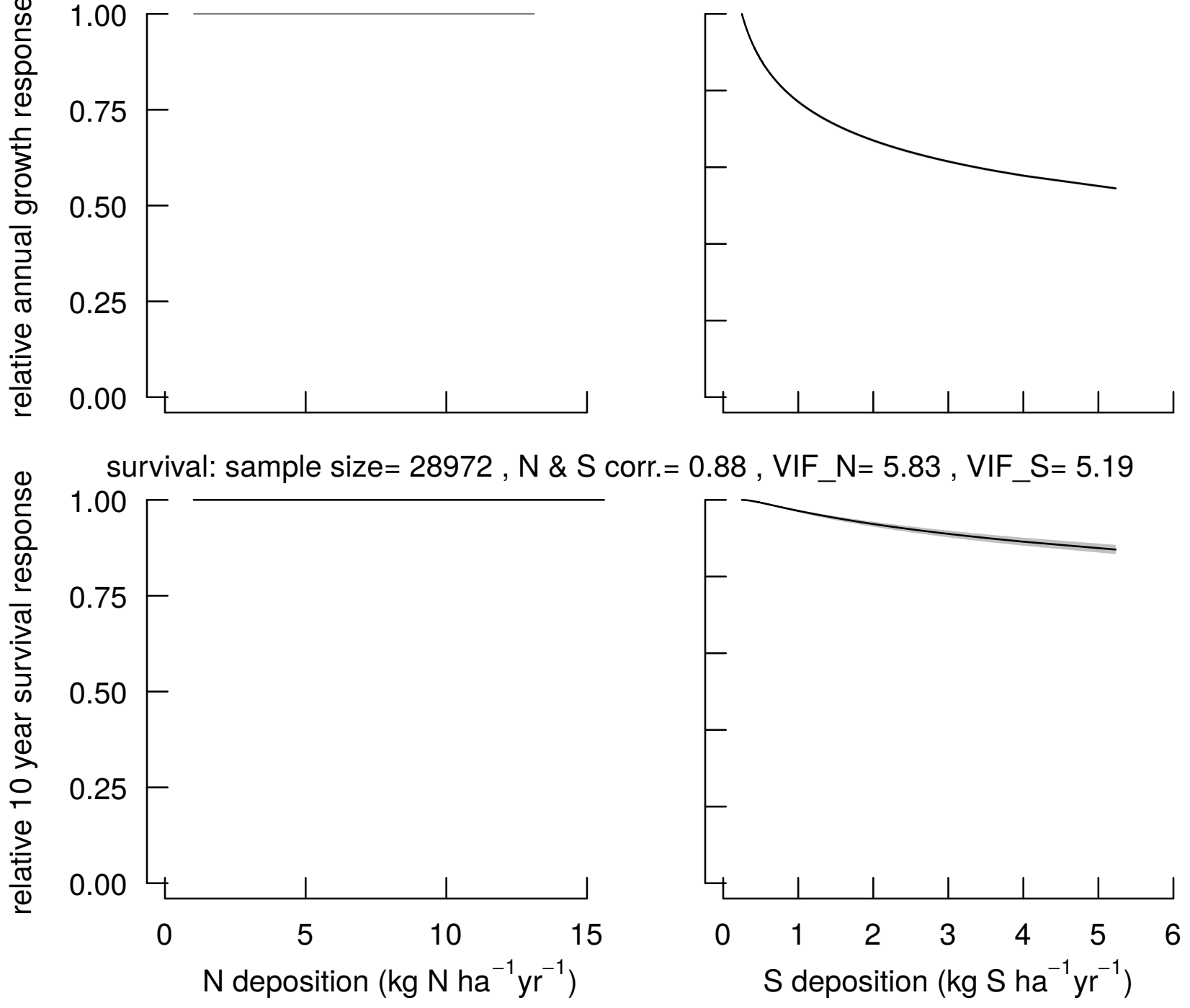


\section{N deposition}

Pinus resinosa

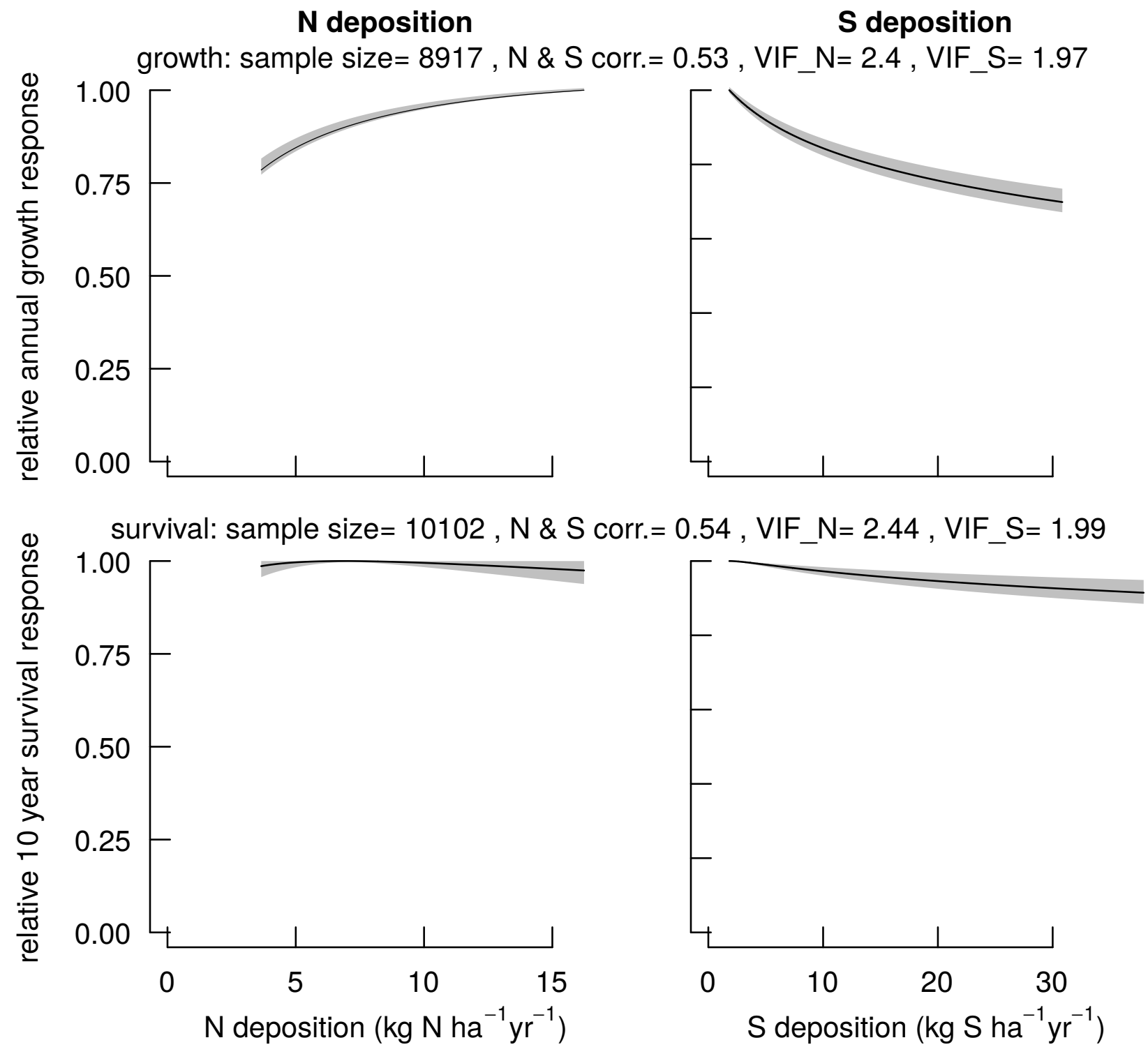




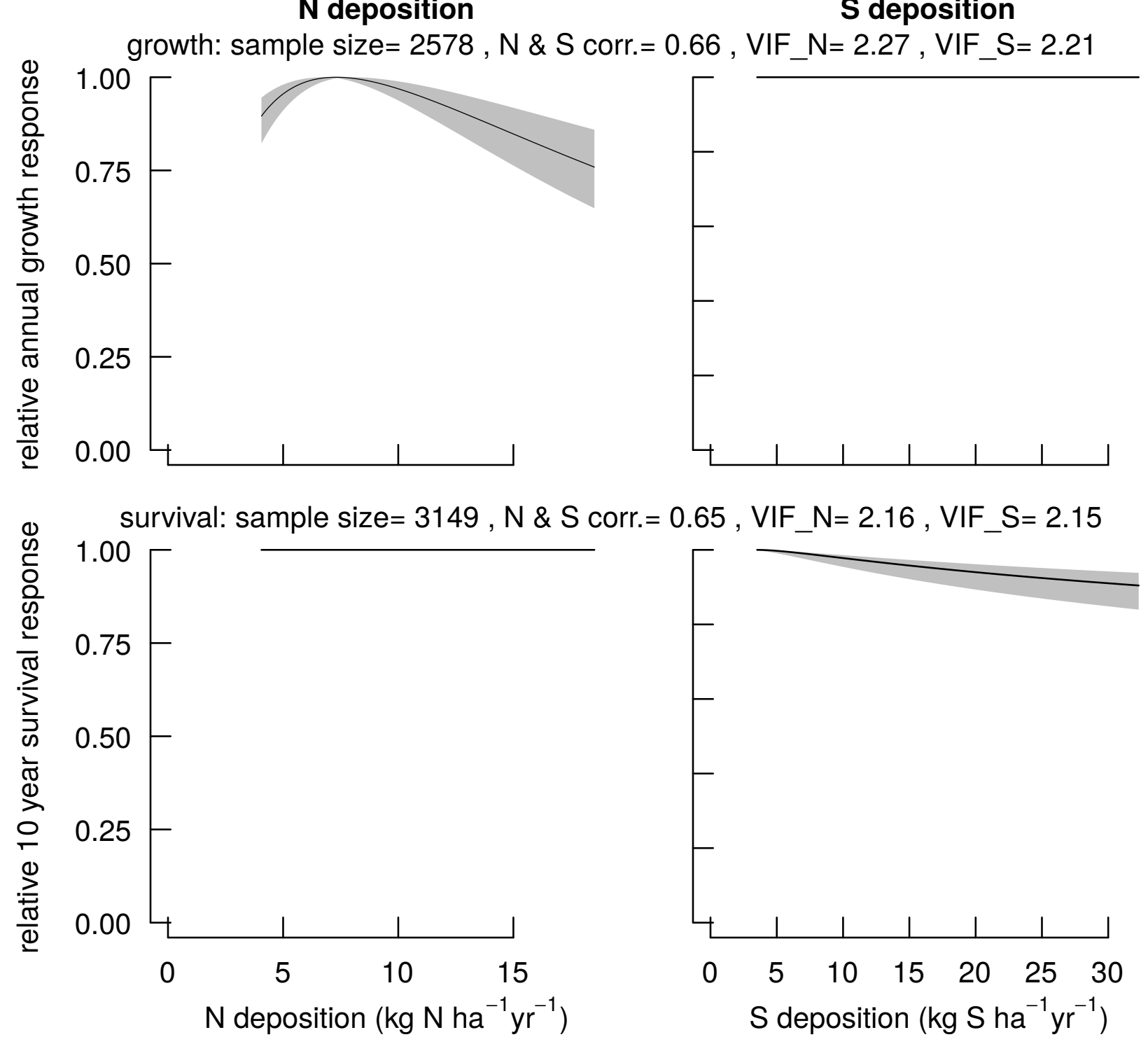




\section{eastern white pine}

N deposition

Pinus strobus

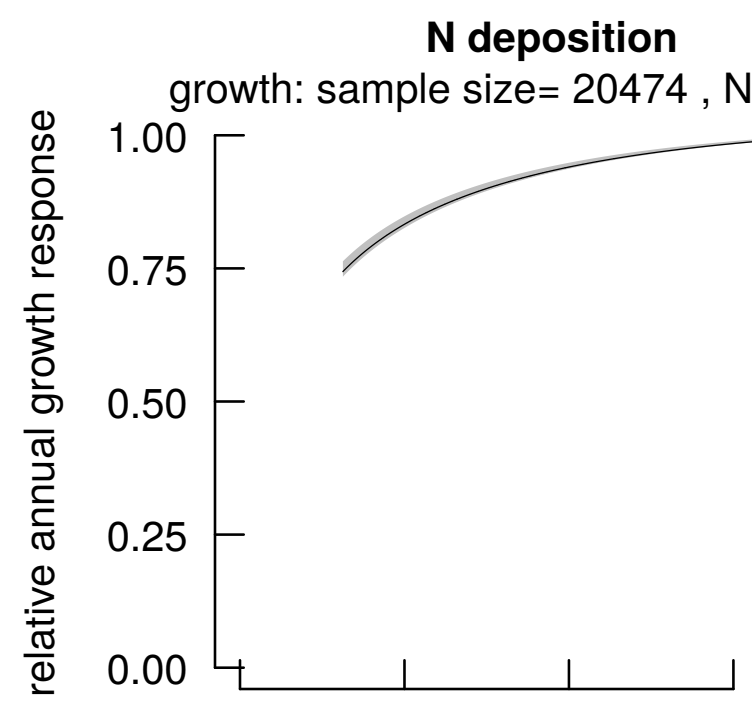

S deposition

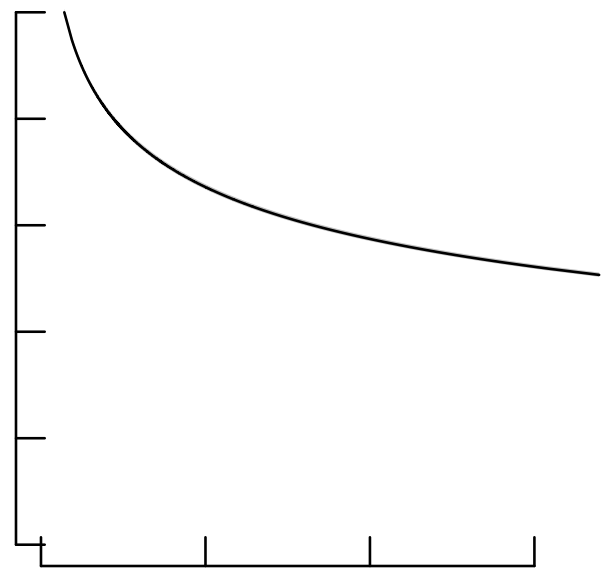

() Survival: sample size $=23371, \mathrm{~N} \& \mathrm{~S}$ corr. $=0.6, \mathrm{VIF} \mathrm{N}=2.15, \mathrm{VIF} \mathrm{S}=1.81$
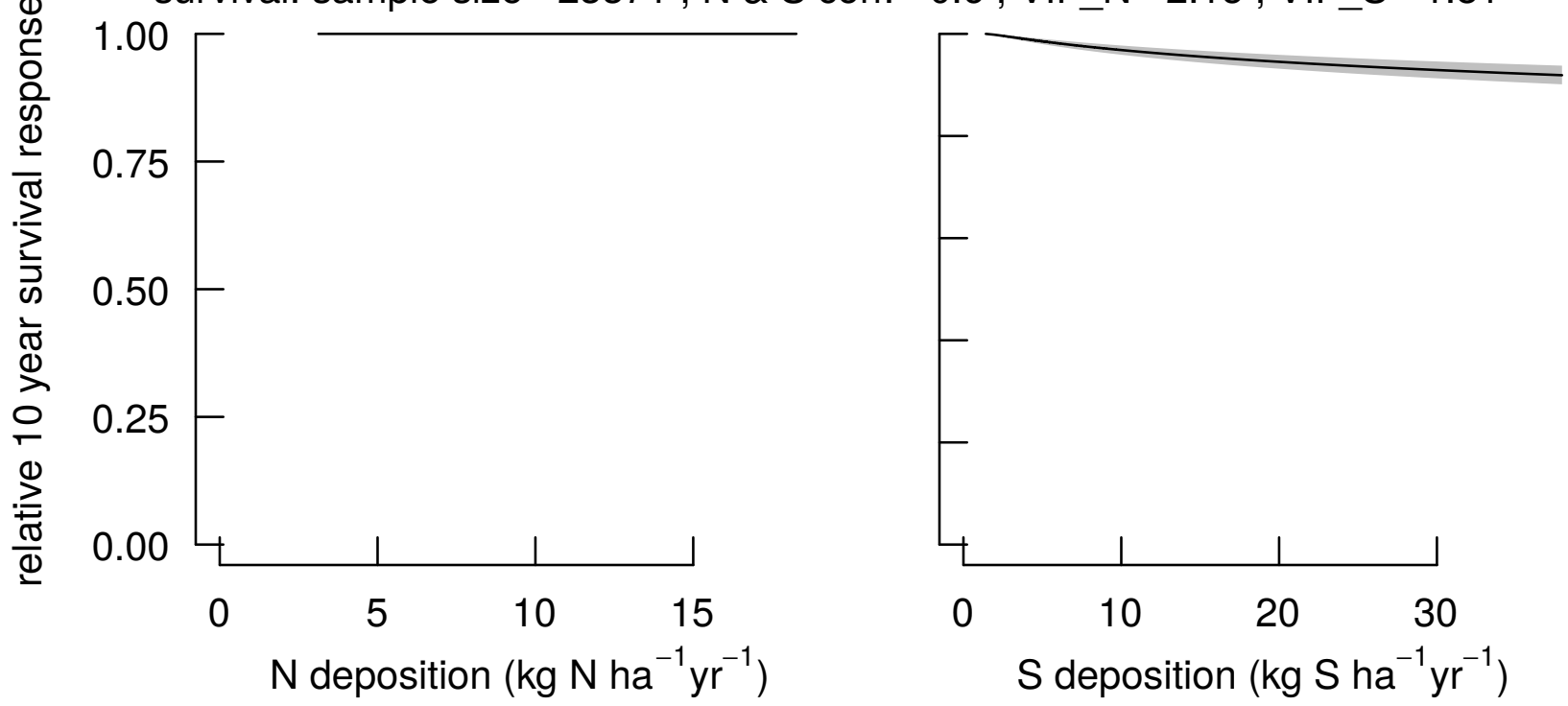


\section{loblolly pine}

N deposition

Pinus taeda
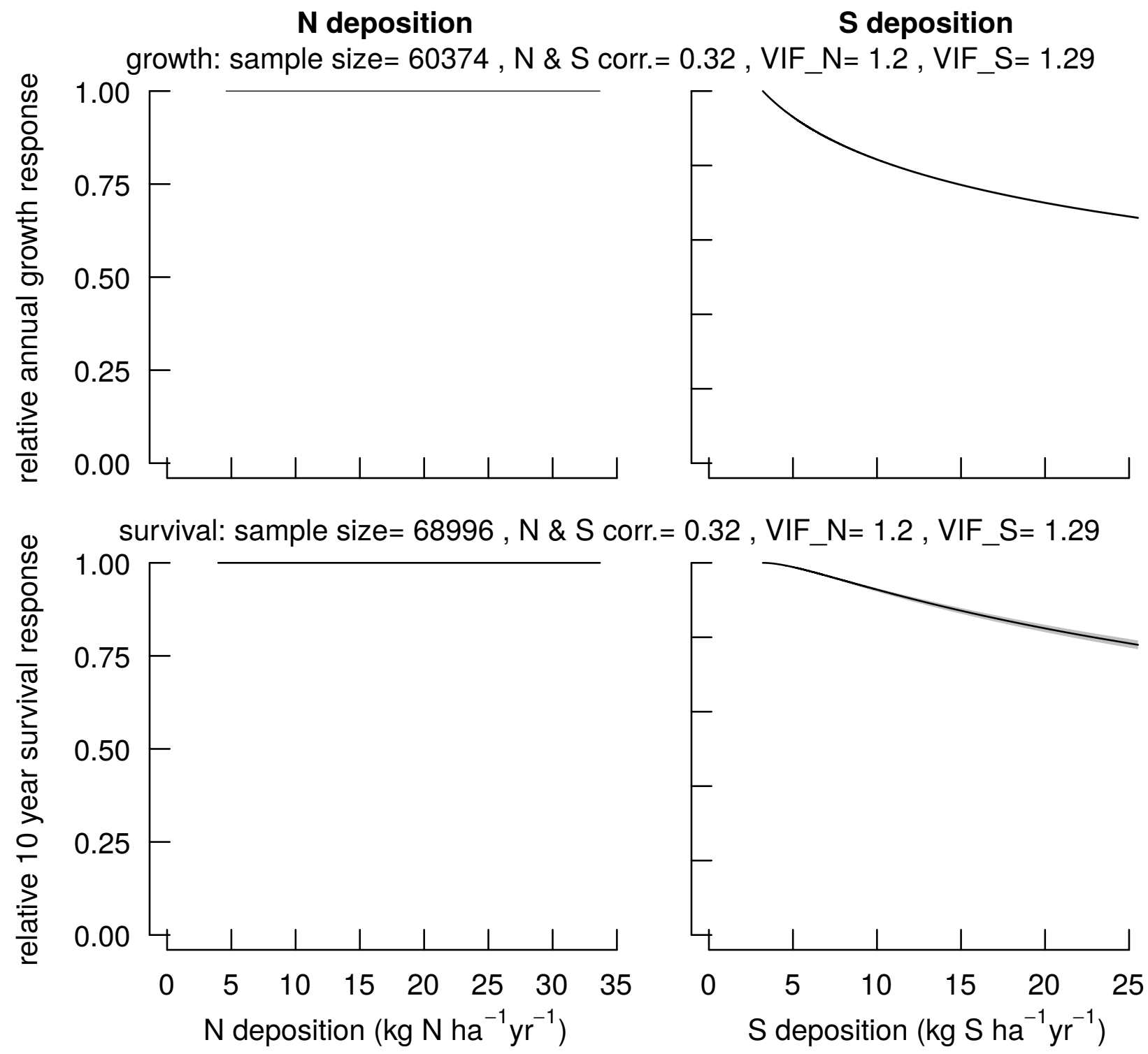


\section{Virginia pine}

N deposition

Pinus virginiana

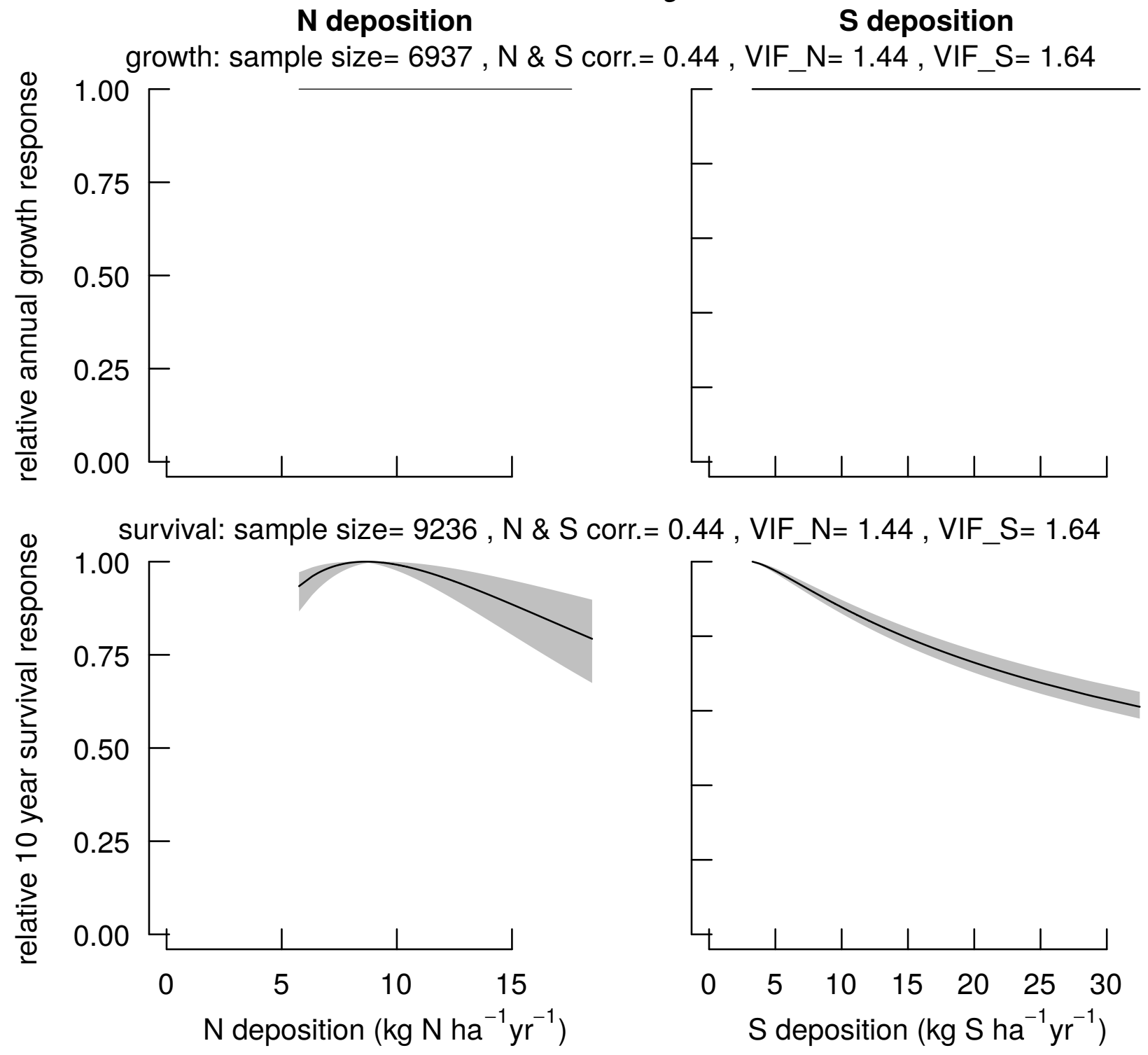




\section{singleleaf pinyon}

$\mathbf{N}$ deposition

Pinus monophylla

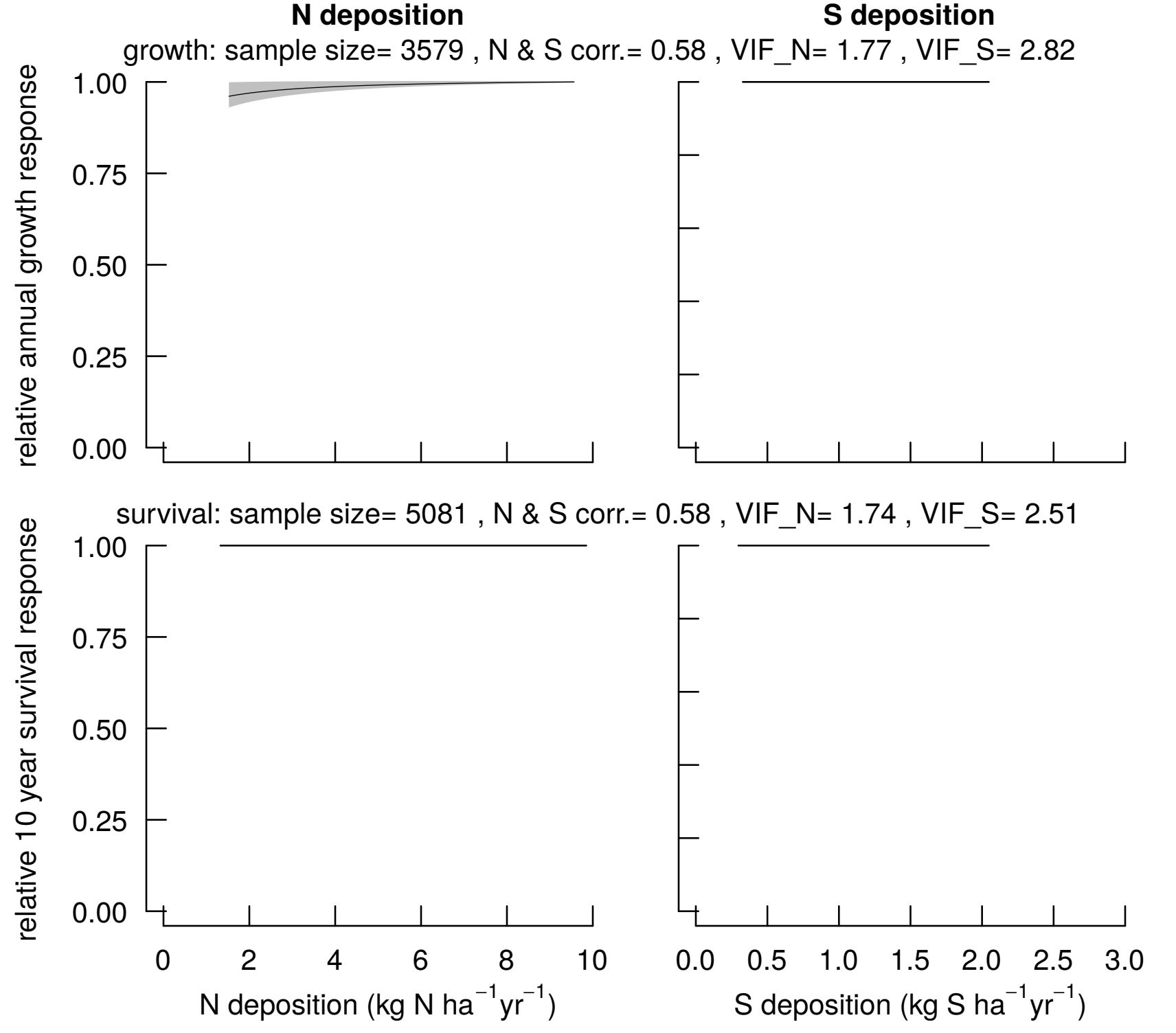




\section{Douglas-fir \\ N deposition \\ Pseudotsuga menziesii}
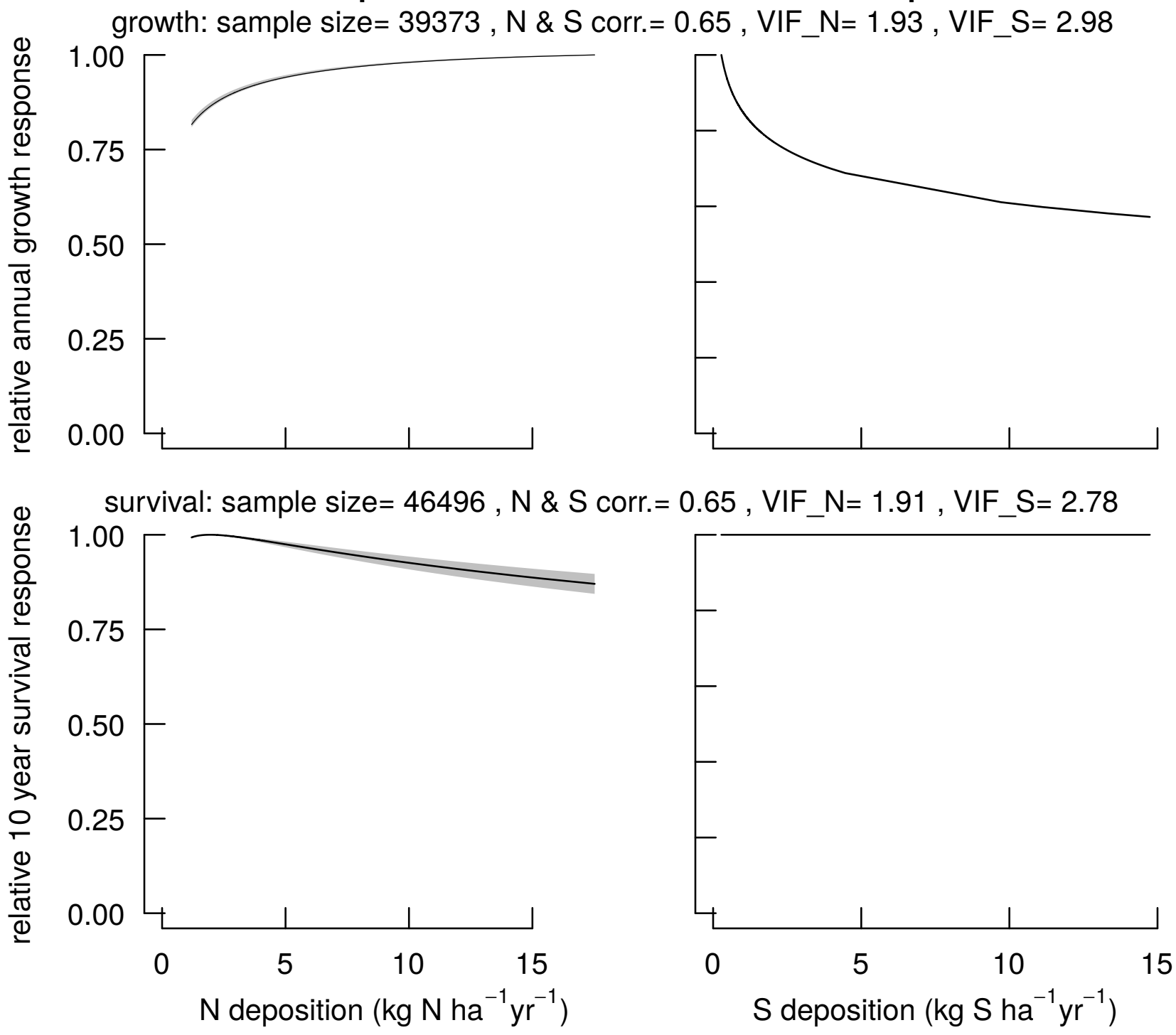


\section{baldcypress}

Taxodium distichum

N deposition

S deposition

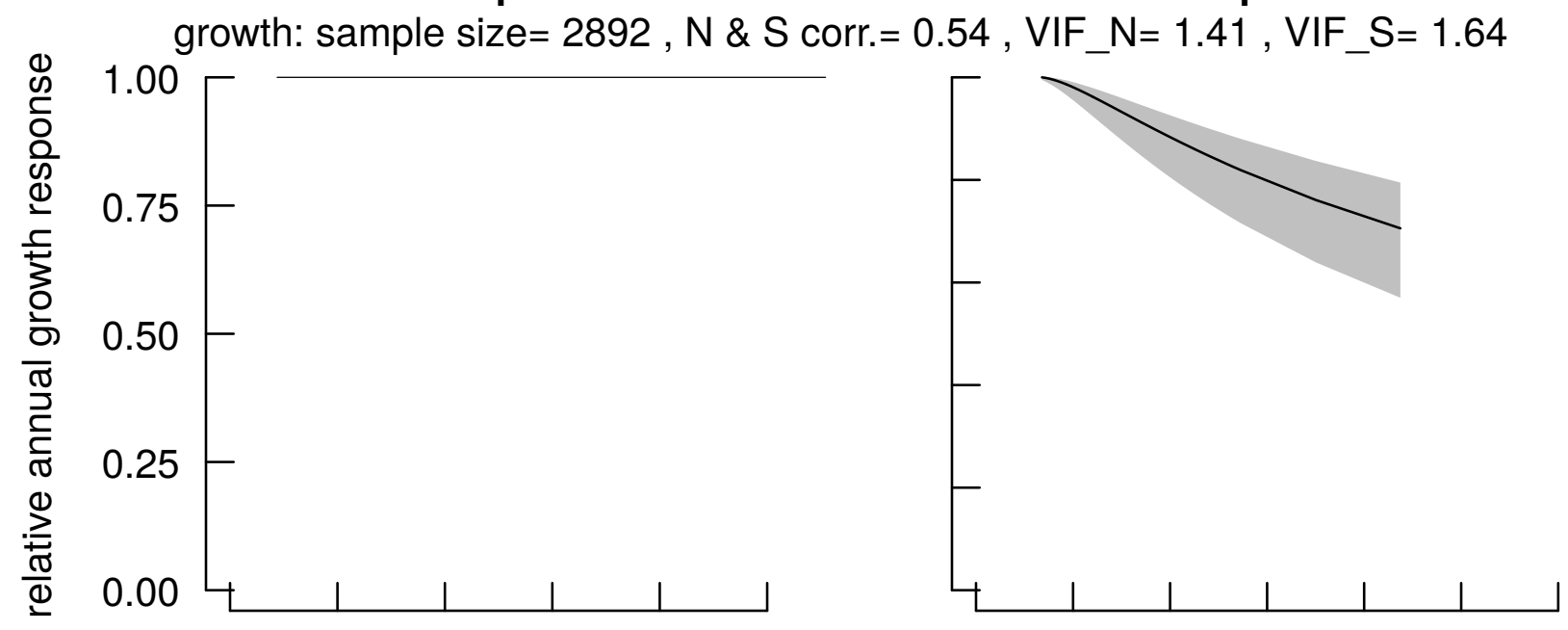

() survival: sample size $=4126, \mathrm{~N} \& \mathrm{~S}$ corr. $=0.55, \mathrm{VIF} N \mathrm{~N}=1.44, \mathrm{VIF} \mathrm{S}=1.66$

D
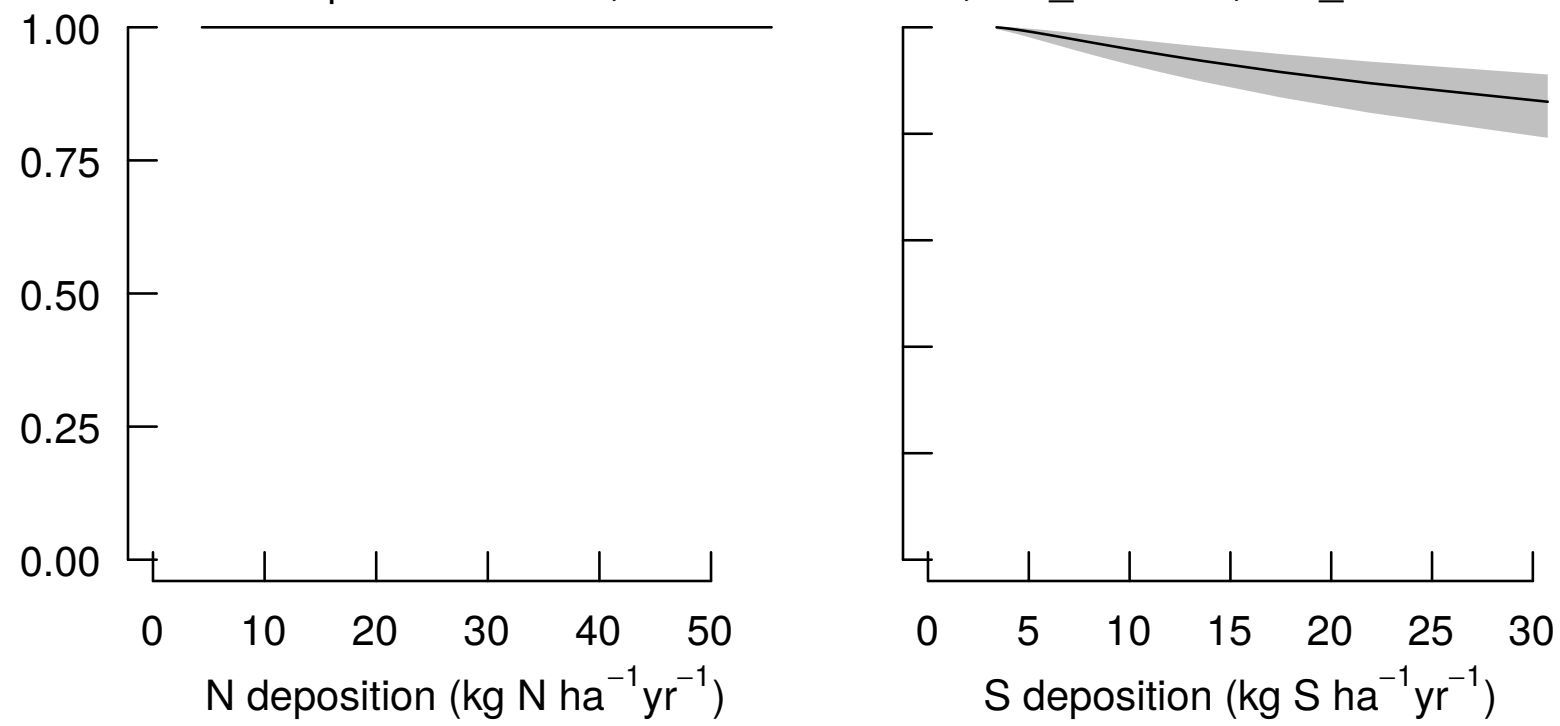


\section{pondcypress}

Taxodium ascendens

\section{N deposition}

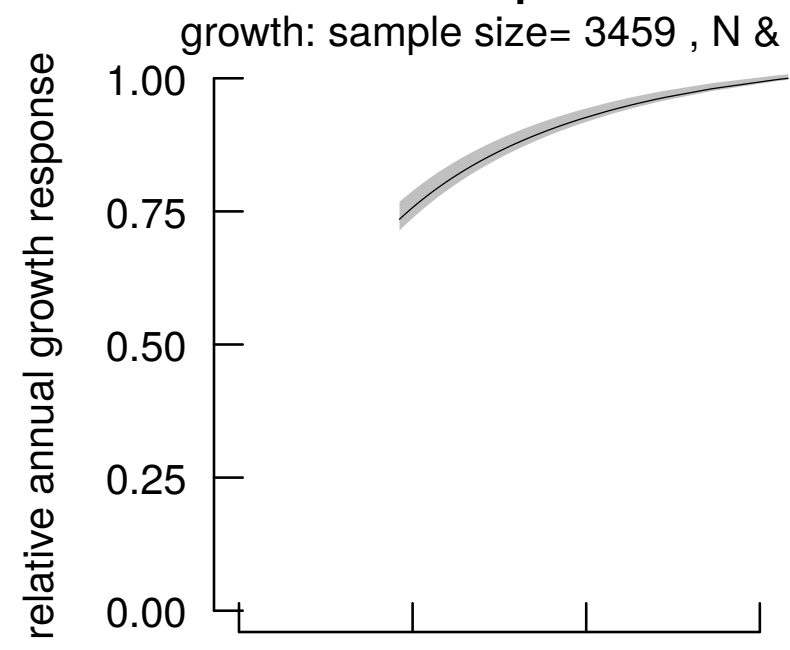

$S$ deposition

$\mathrm{N}=2.06$, VIF_S $=2.12$

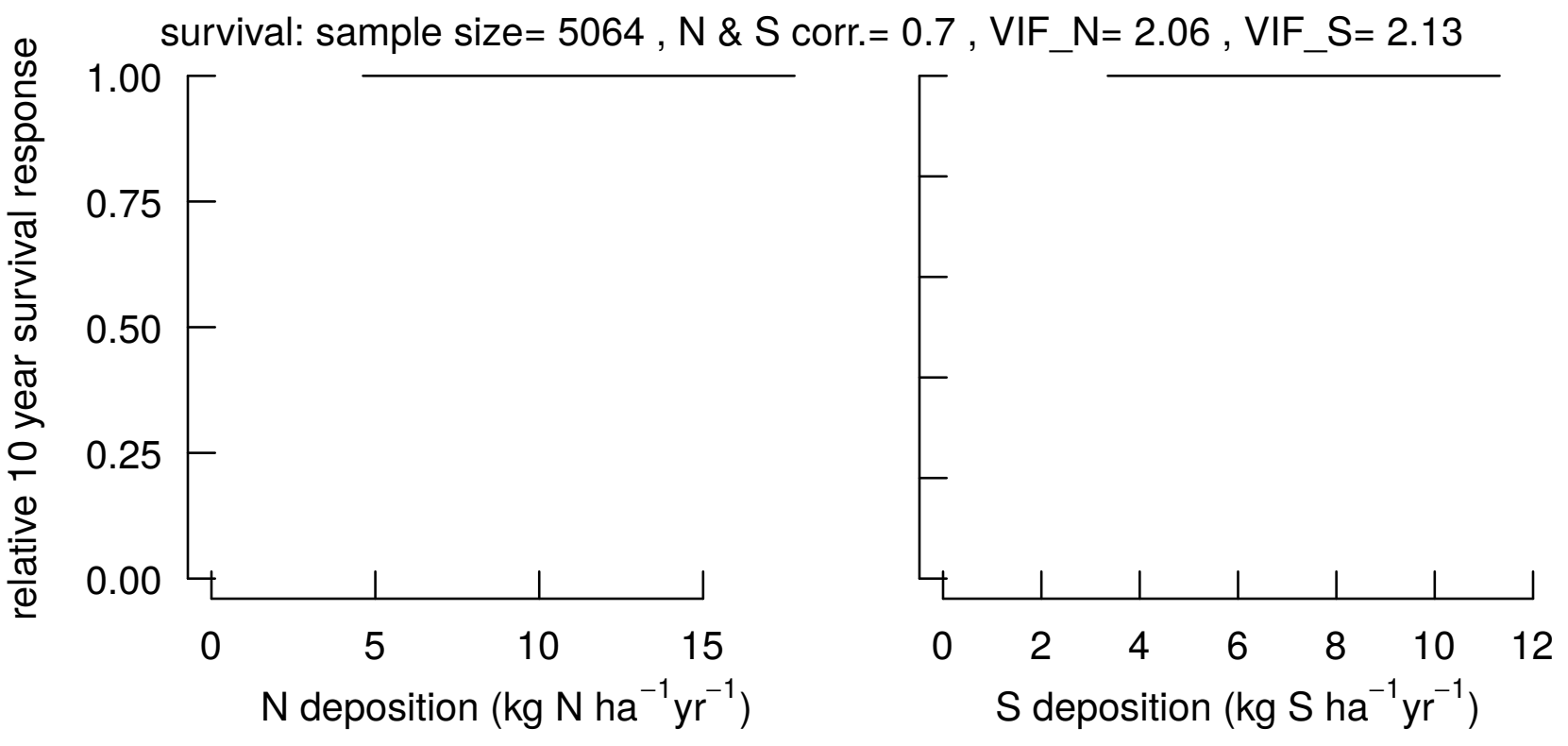




\section{northern white-cedar}

N deposition

Thuja occidentalis

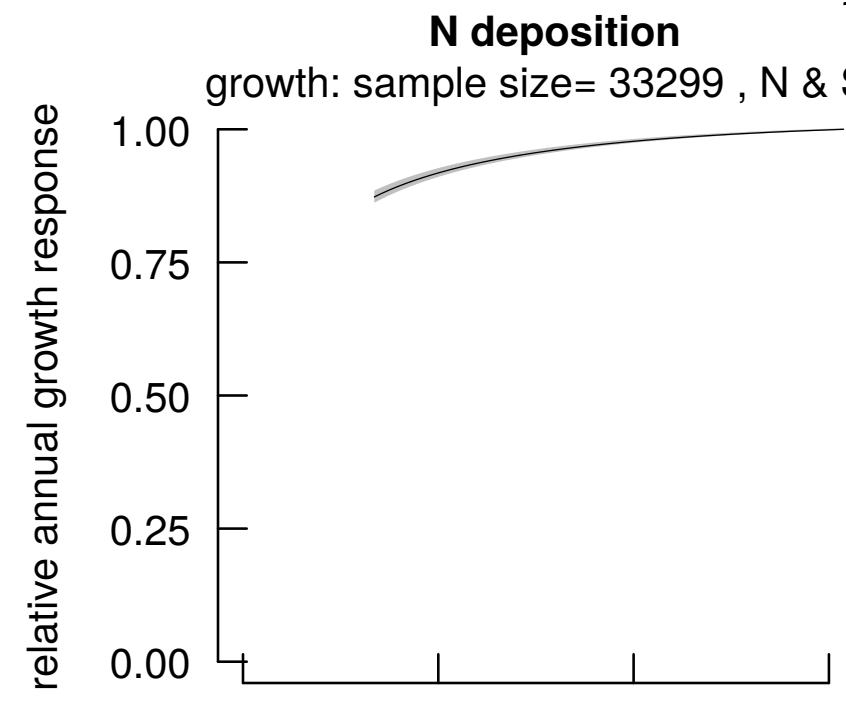

$S$ deposition
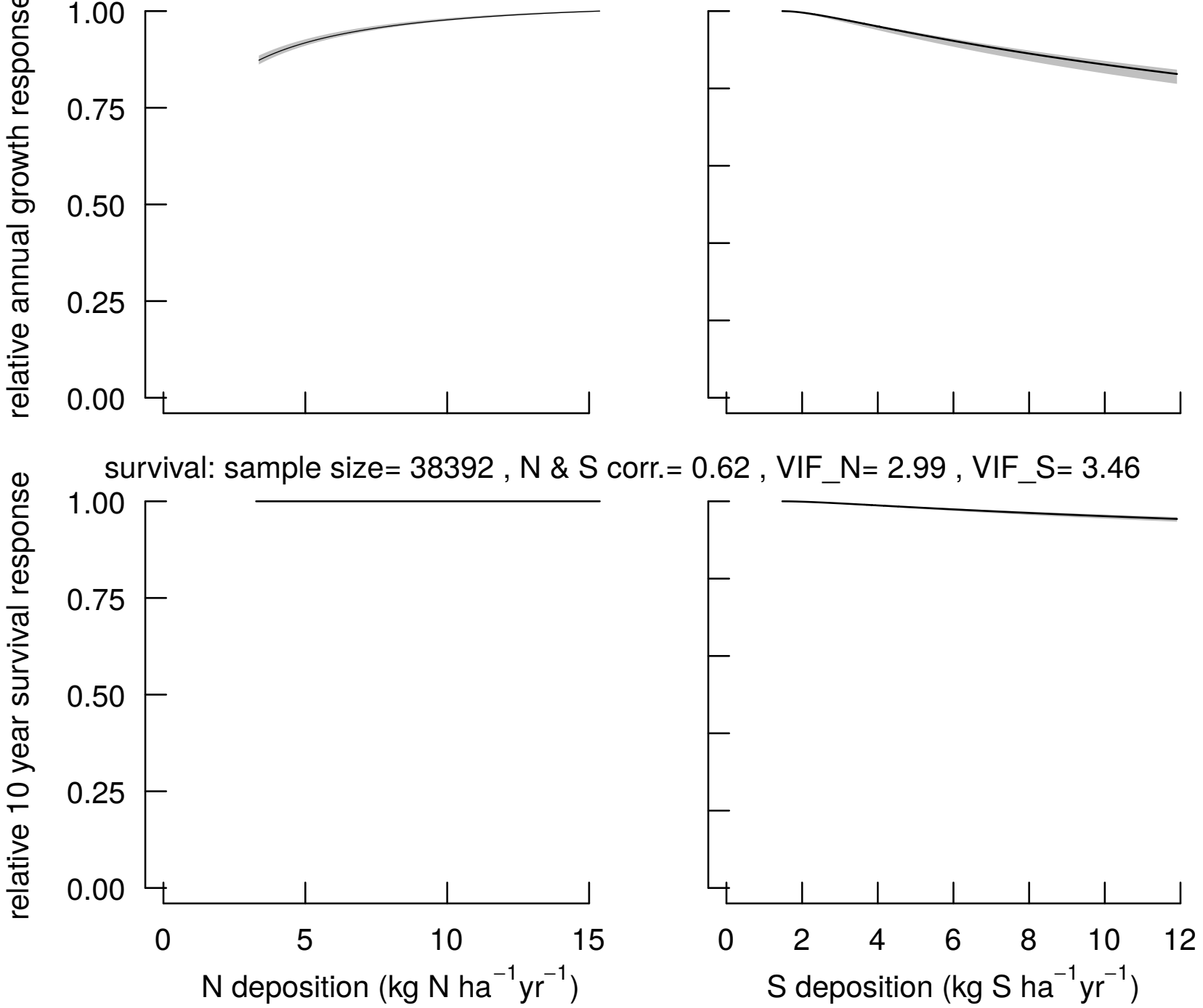


\section{western redcedar}

N deposition

Thuja plicata

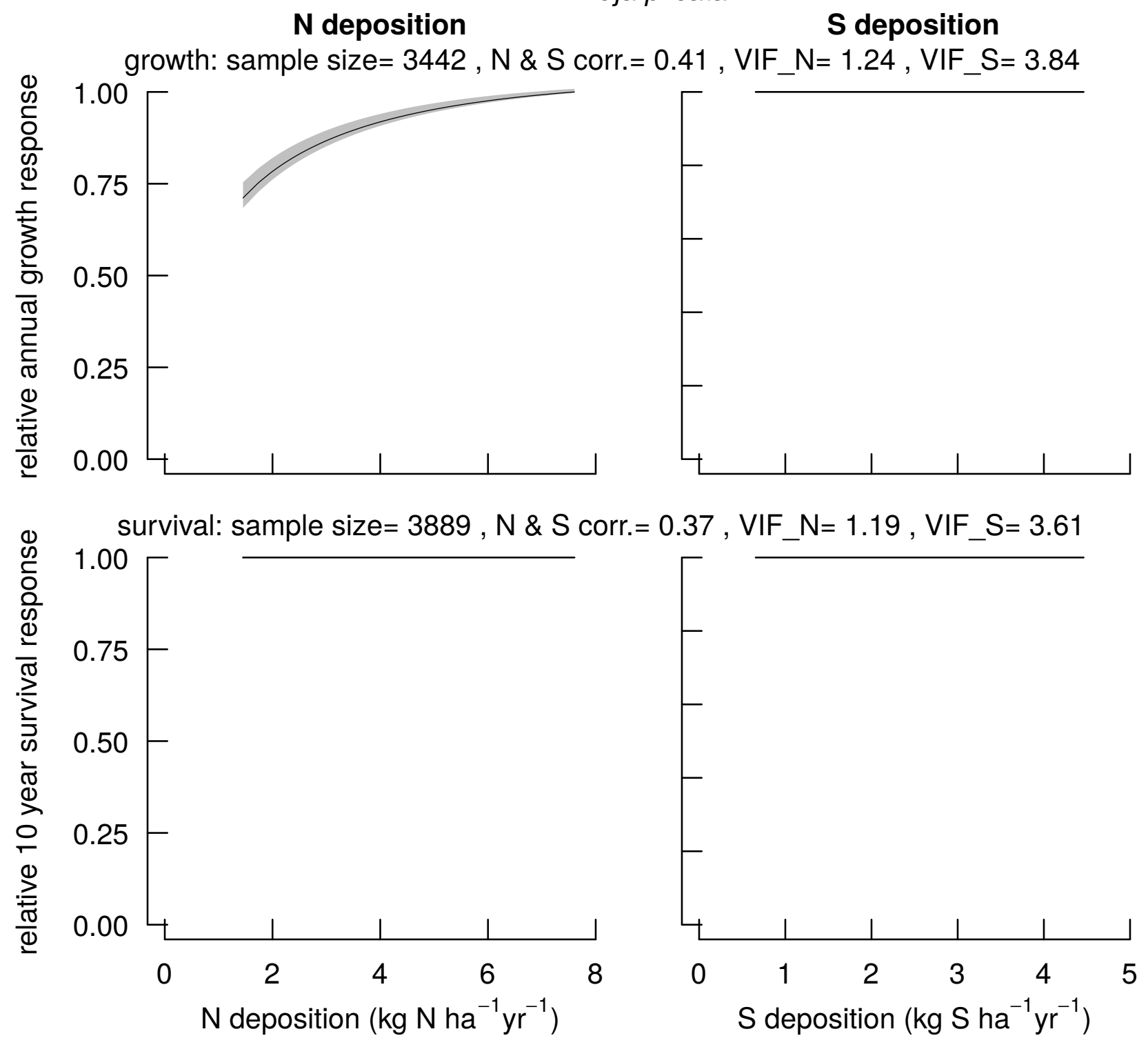




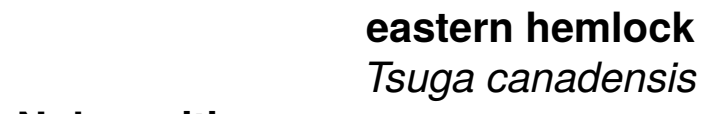

$\mathrm{N}$ deposition

Tsuga canadensis
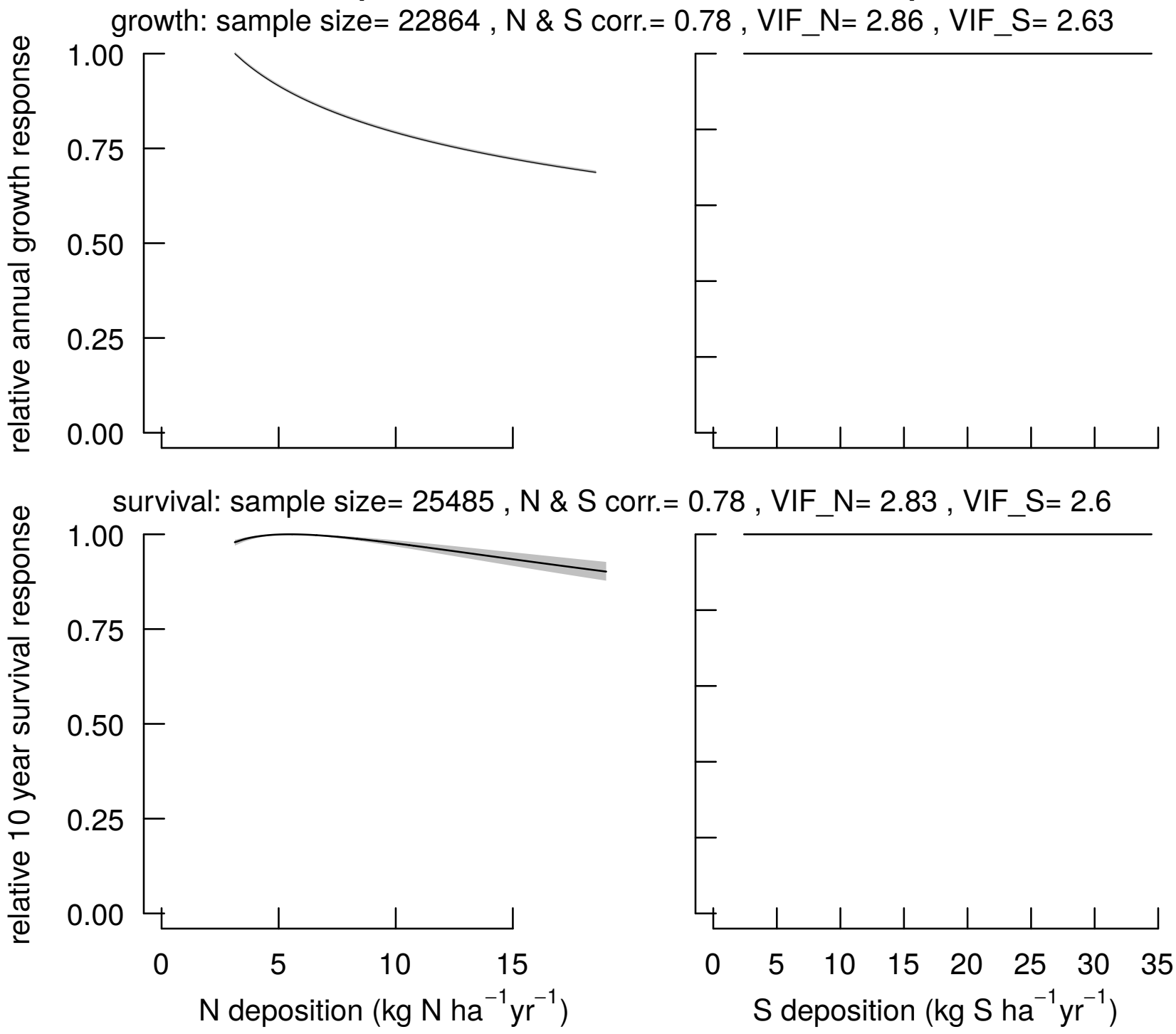


\section{western hemlock}

N deposition

Tsuga heterophylla
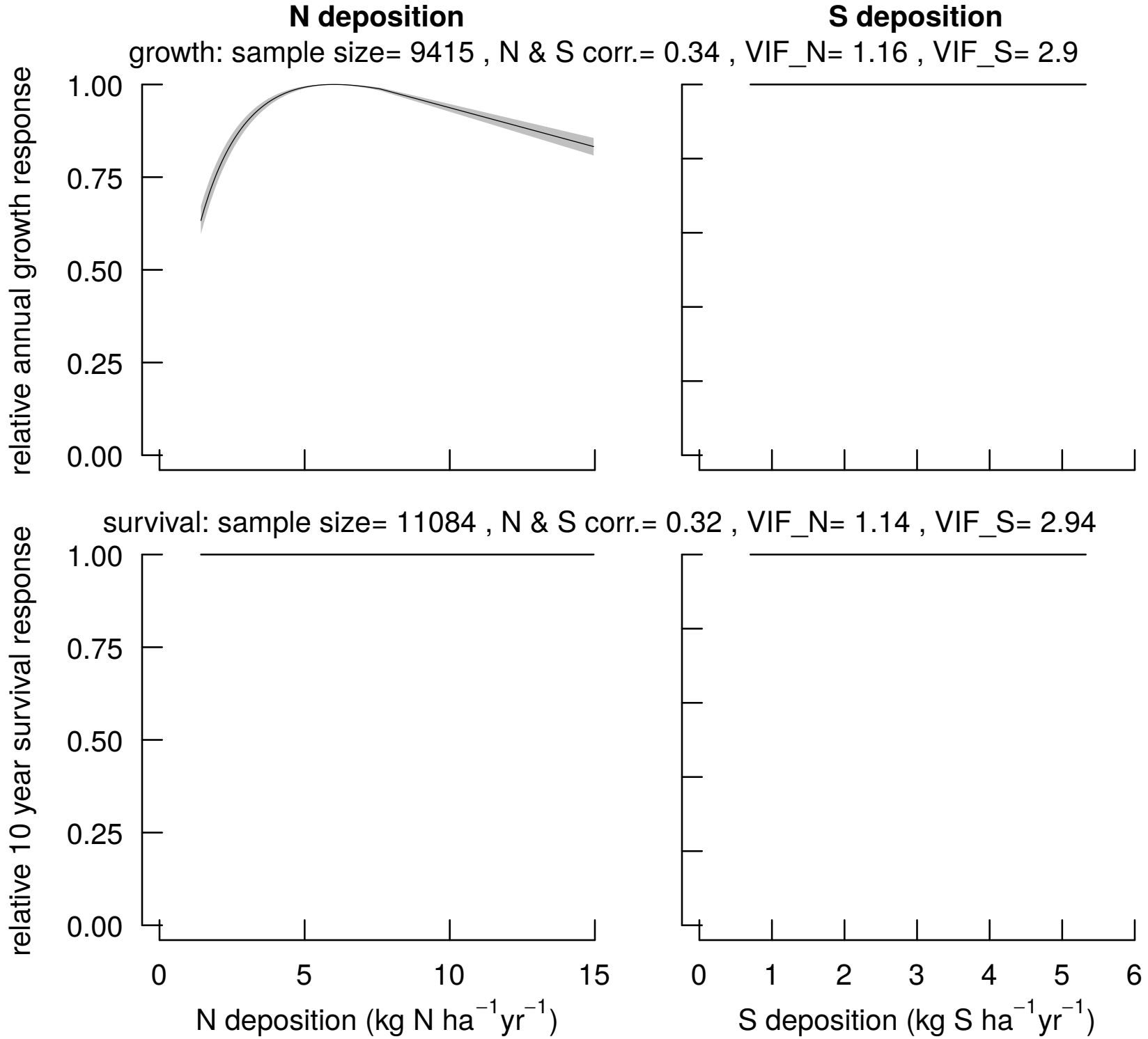


\section{mountain hemlock}

N deposition

Tsuga mertensiana

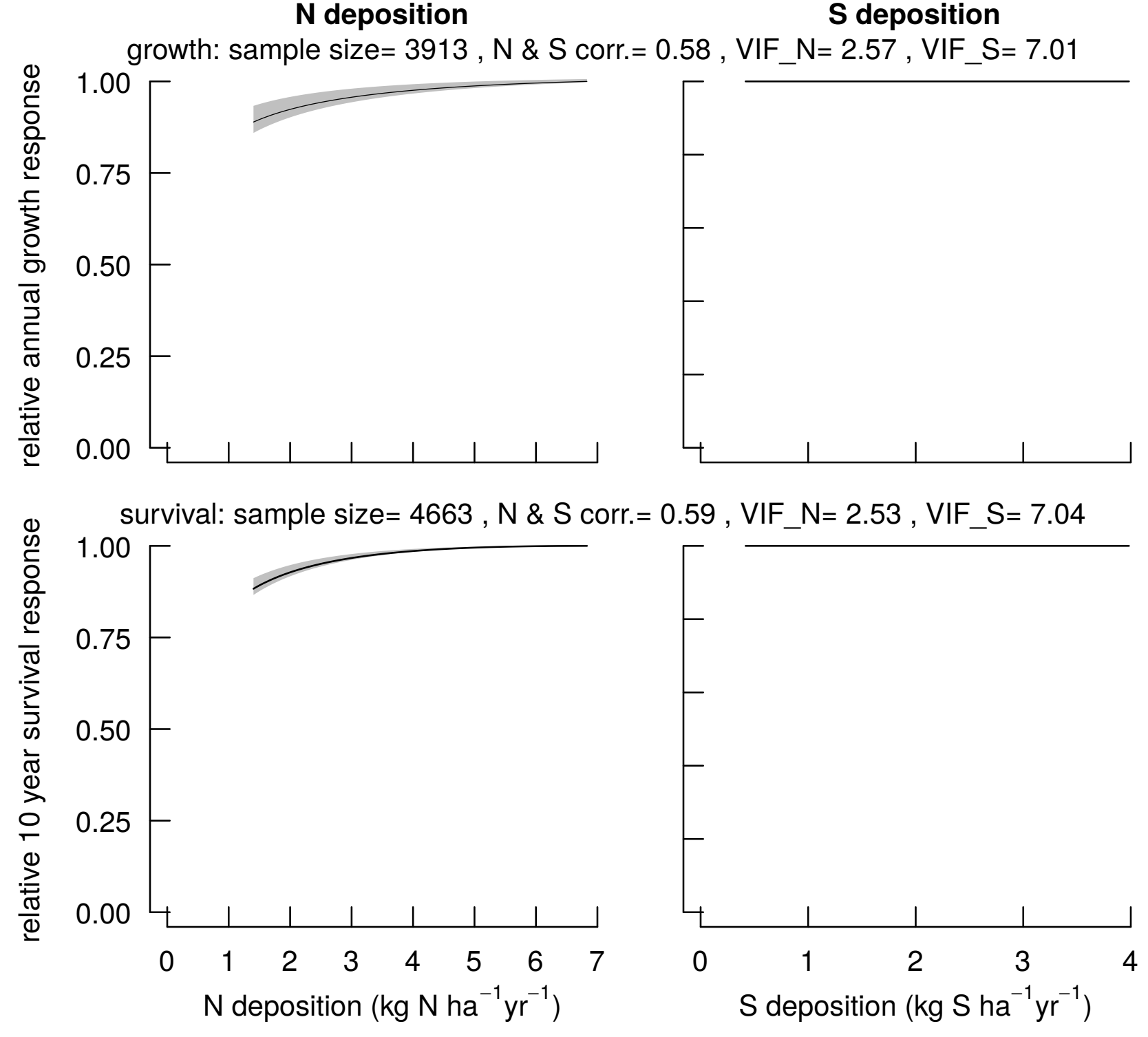




\section{boxelder}

N deposition

Acer negundo

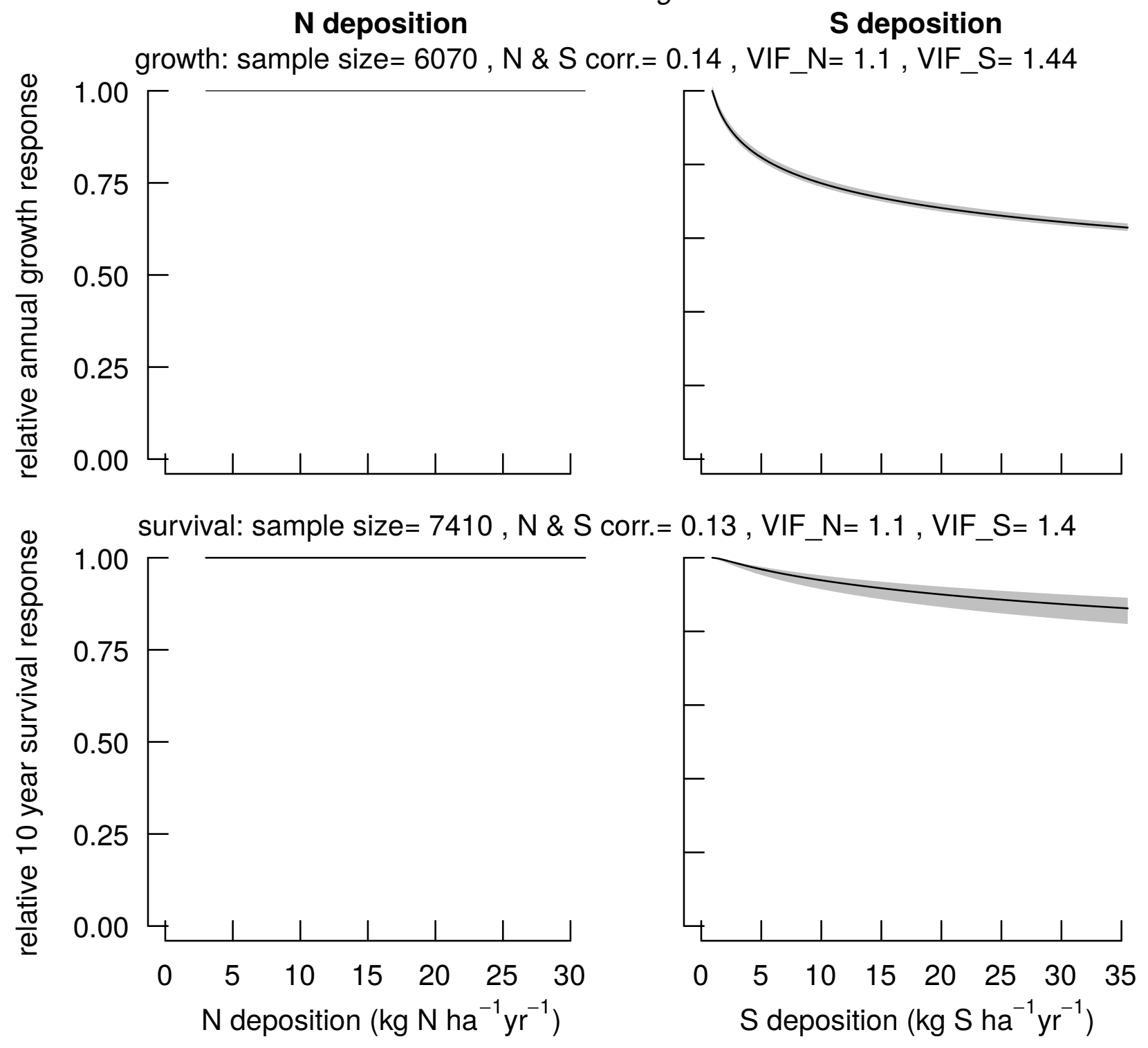




\section{red maple}

Acer rubrum

\section{N deposition}
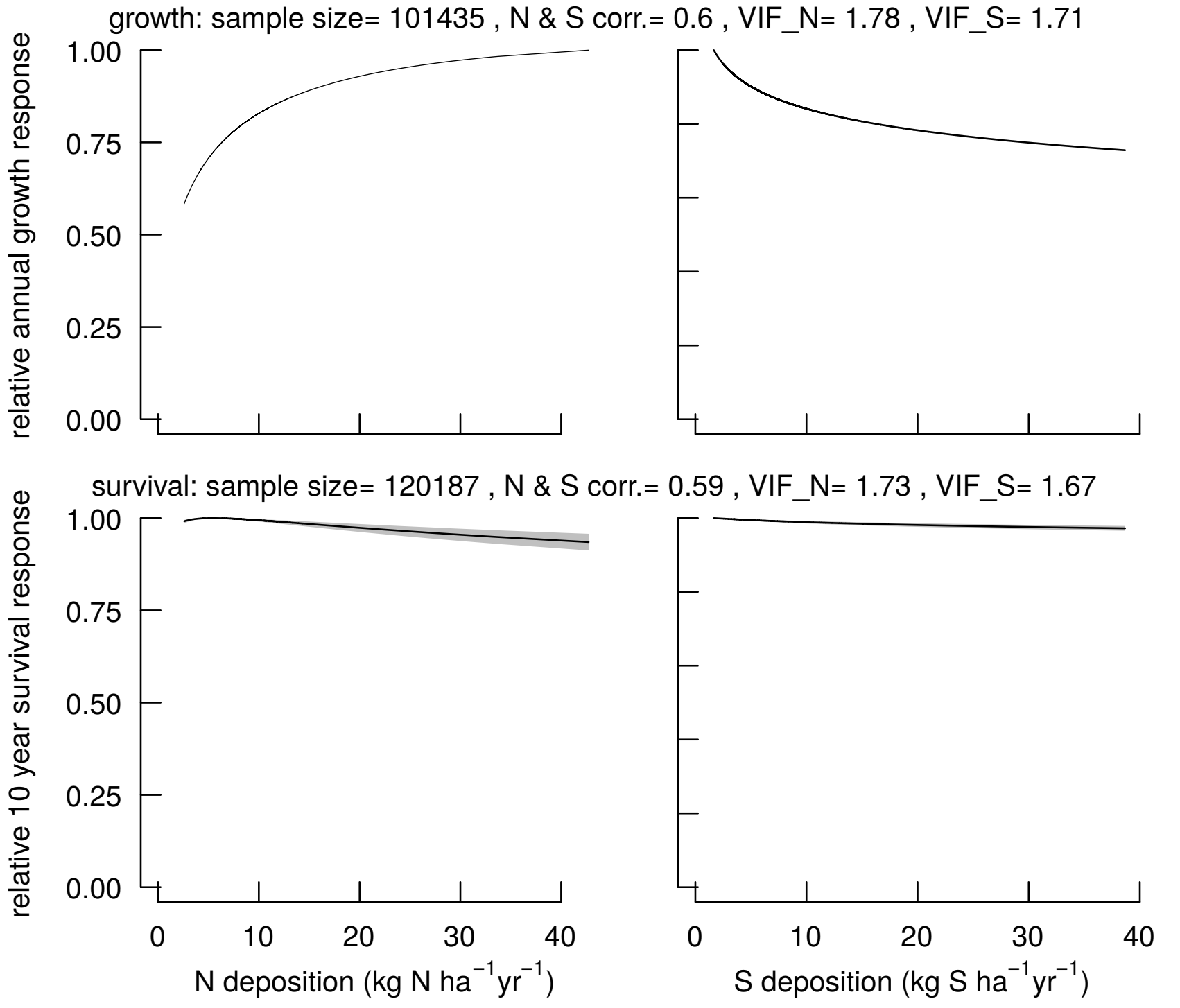


\section{silver maple}

Acer saccharinum

\section{N deposition}

$S$ deposition
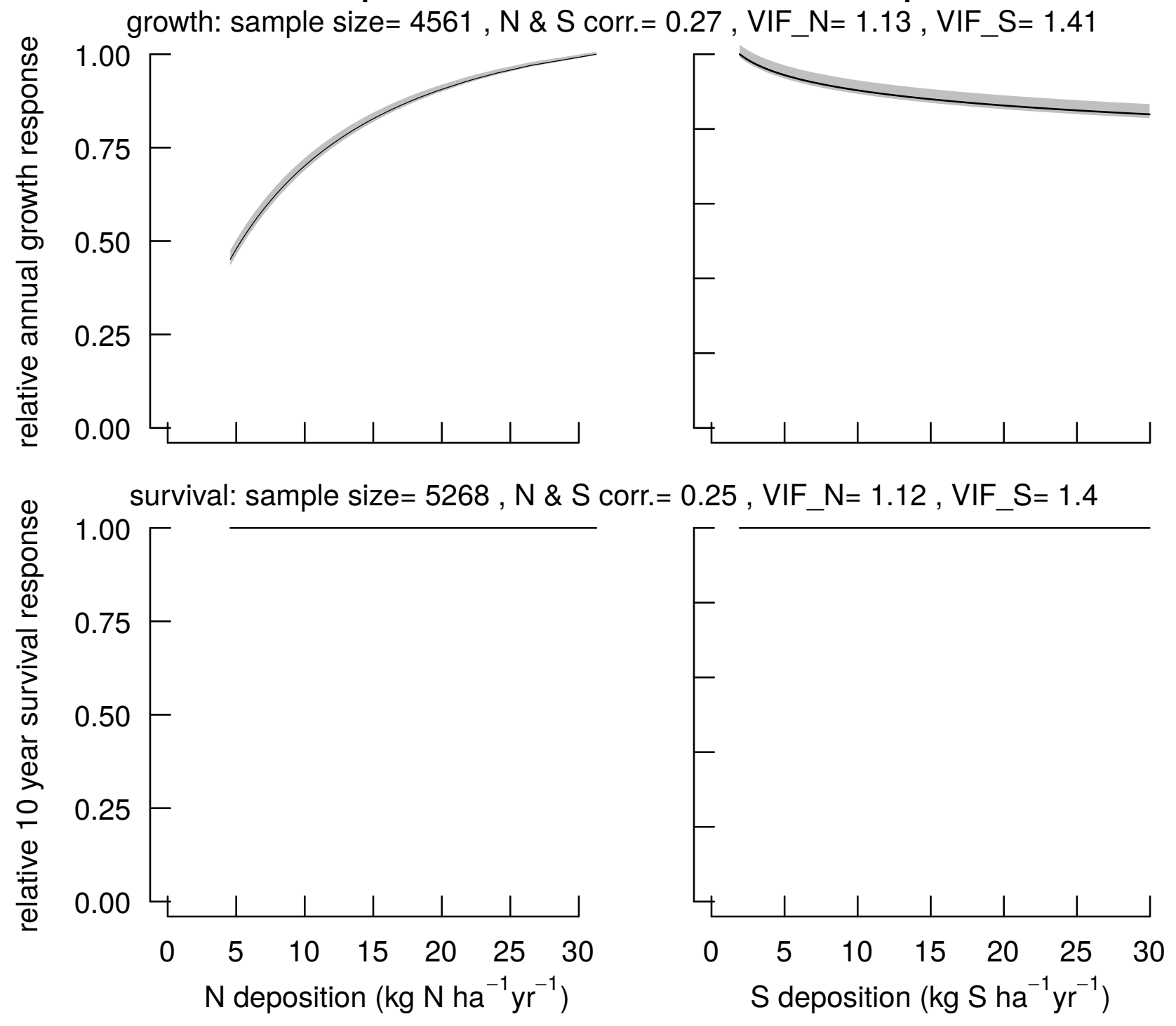

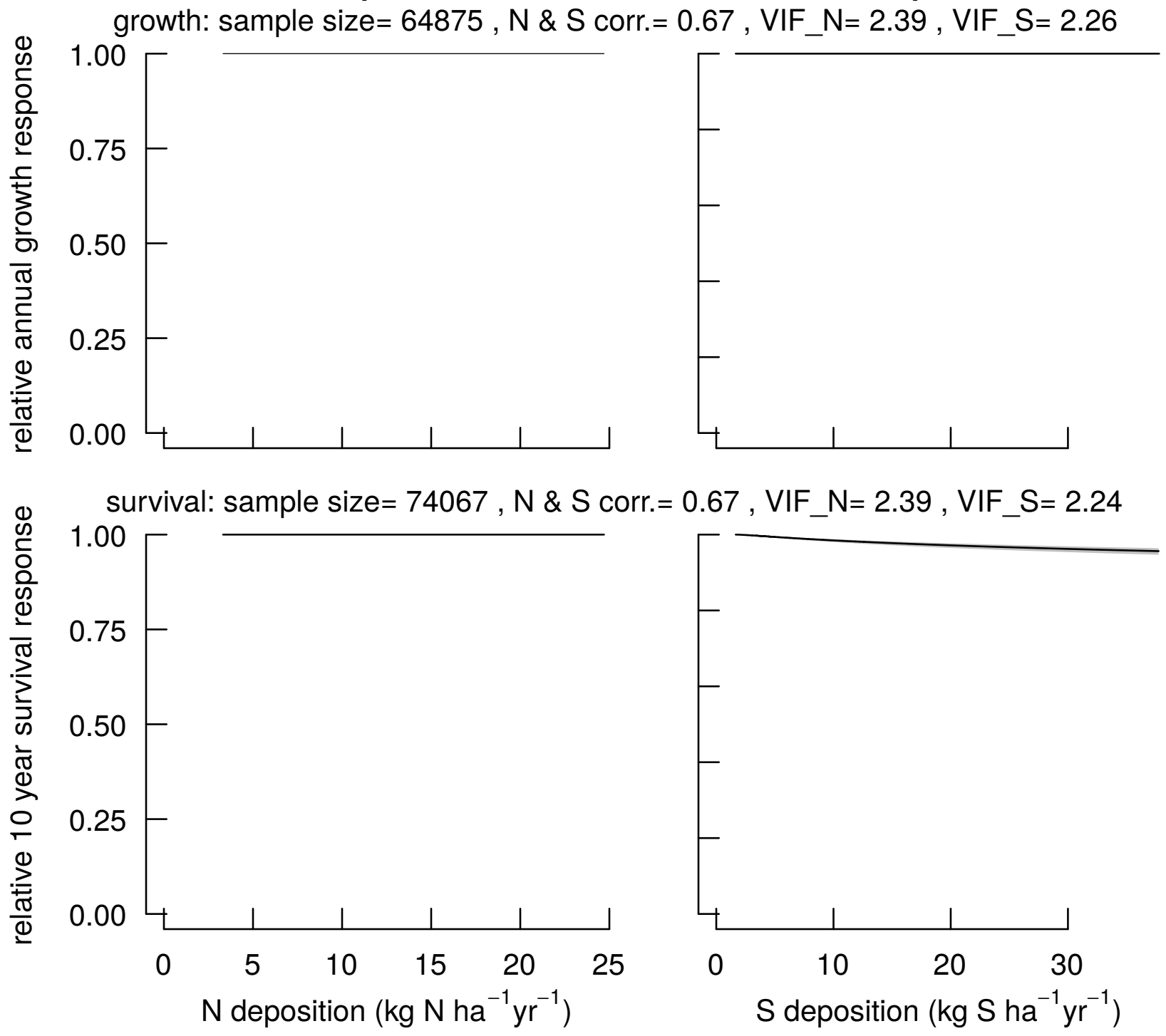


\section{yellow birch}

N deposition

Betula alleghaniensis
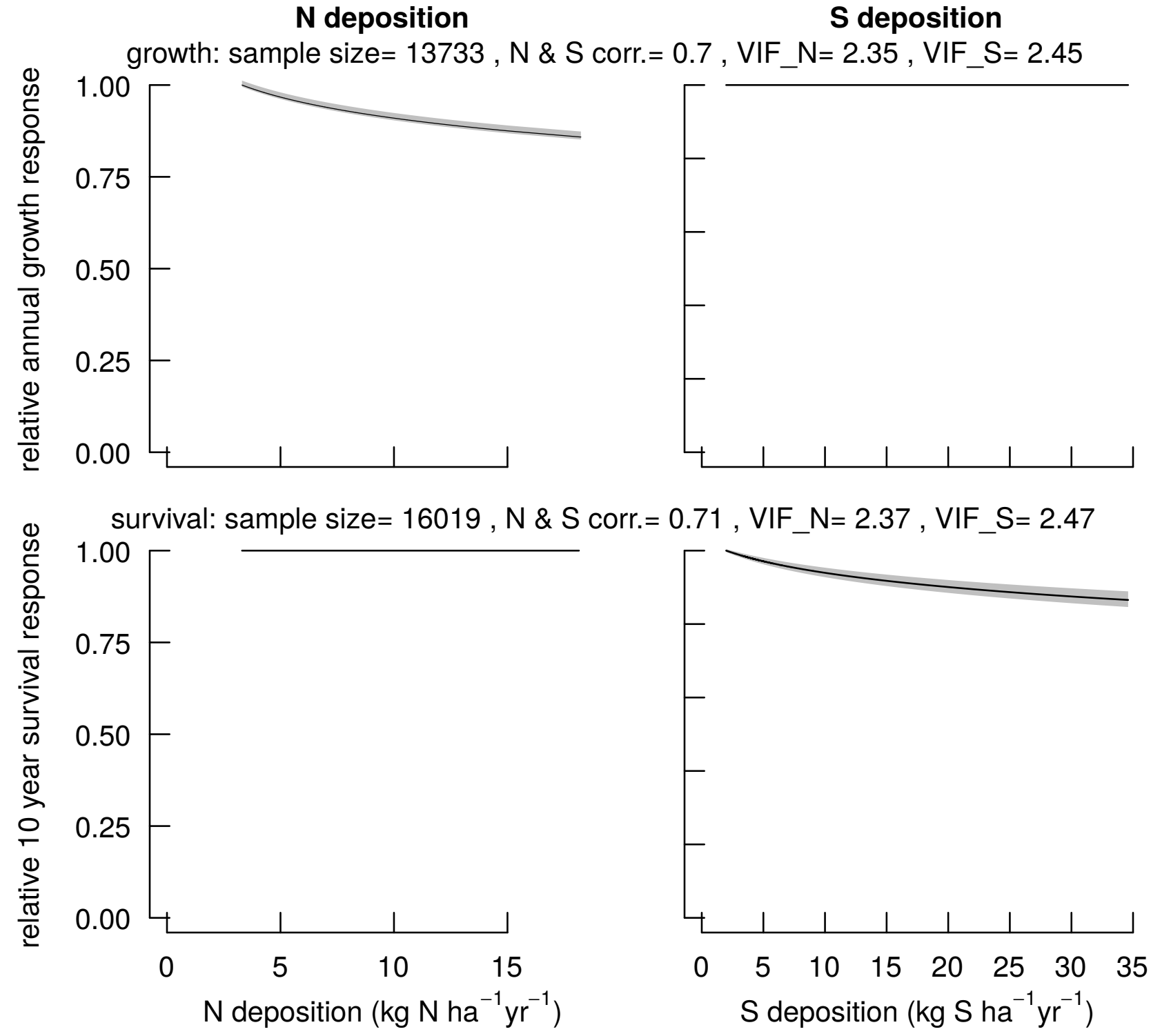


\section{sweet birch}

$\mathbf{N}$ deposition

Betula lenta
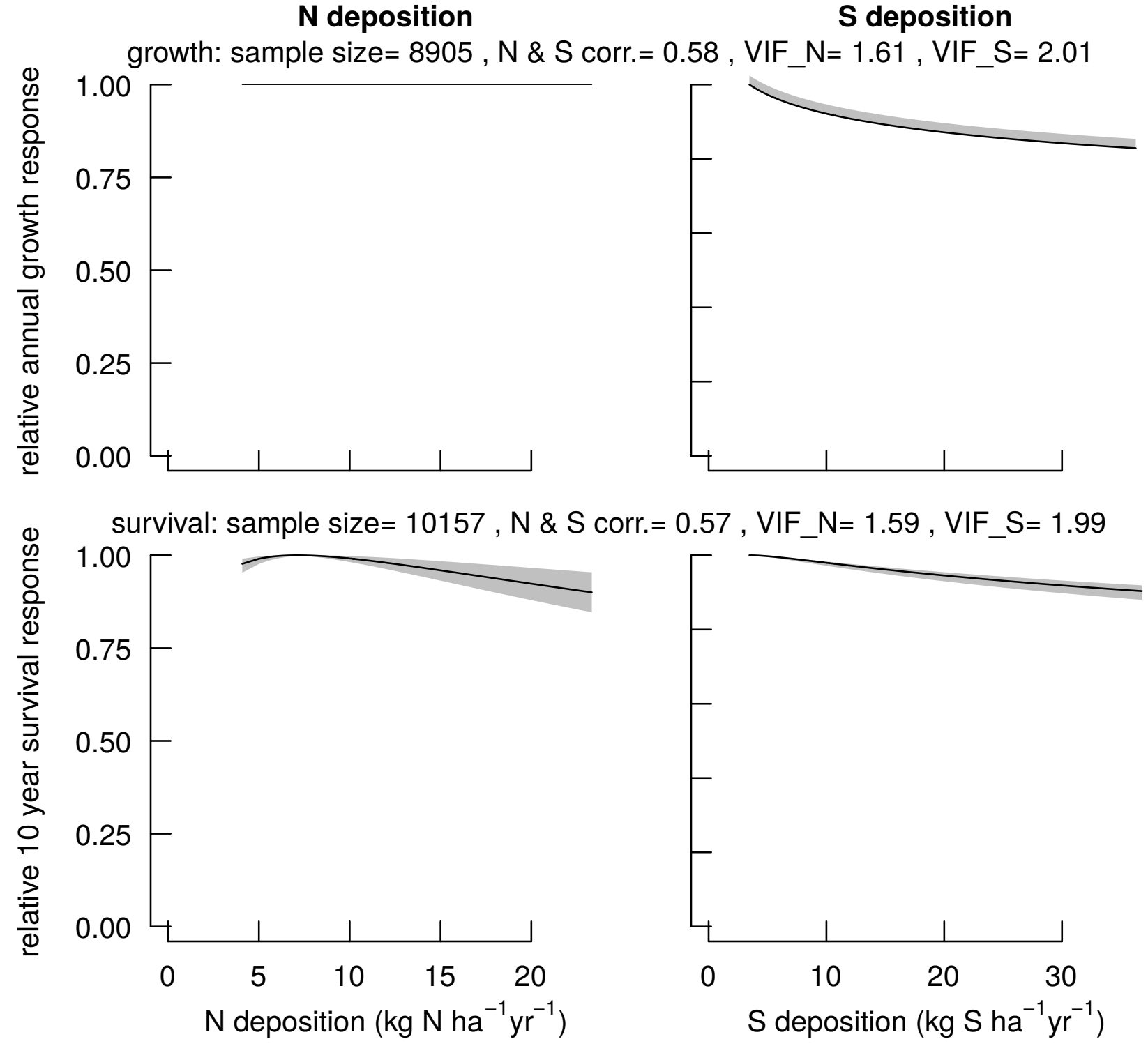


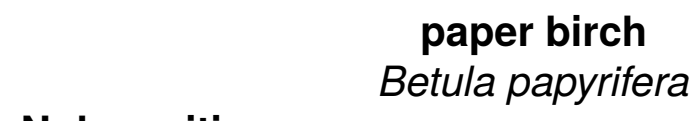

$\mathrm{N}$ deposition

Betula papyrifera

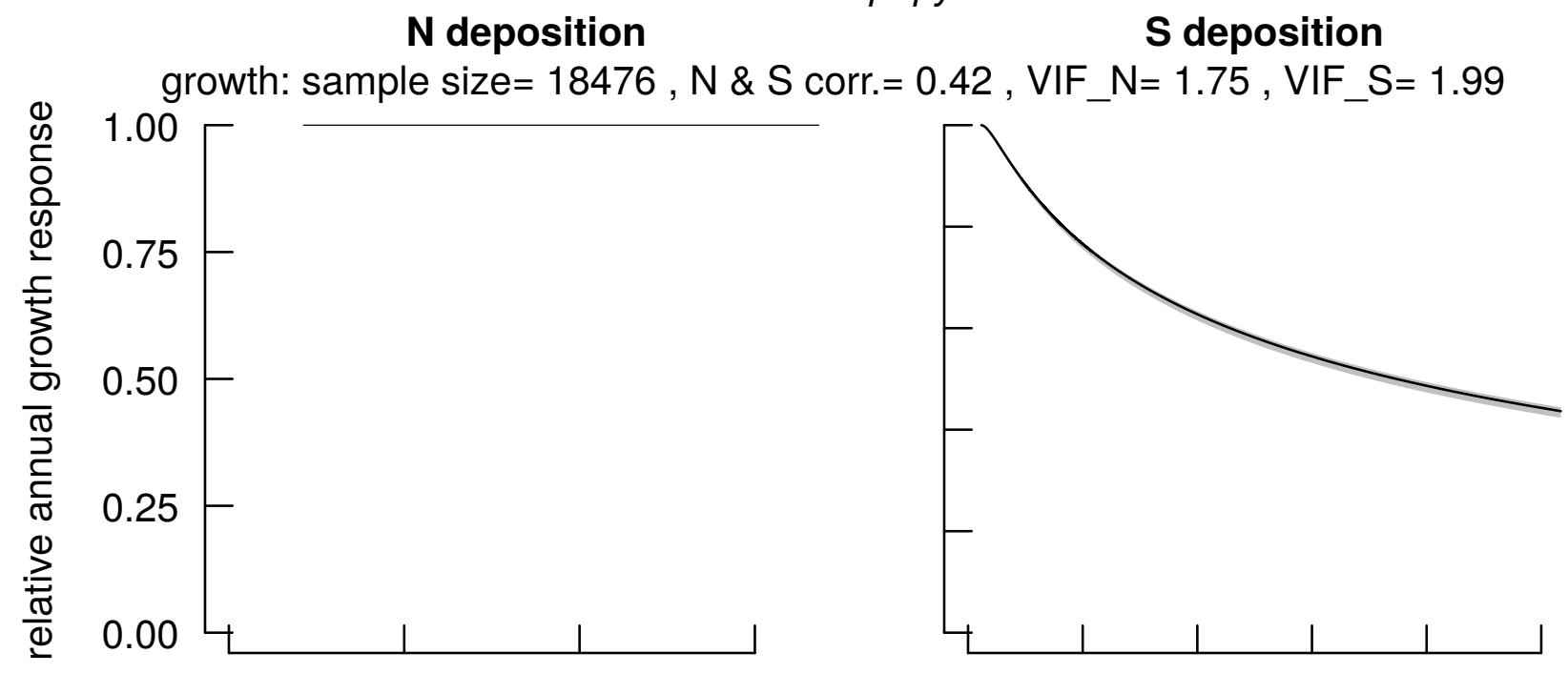

(1) survival: sample size $=24432, \mathrm{~N} \& \mathrm{~S}$ corr. $=0.42, \mathrm{VIF} \mathrm{N}=1.71, \mathrm{VIF} \mathrm{S}=2.02$
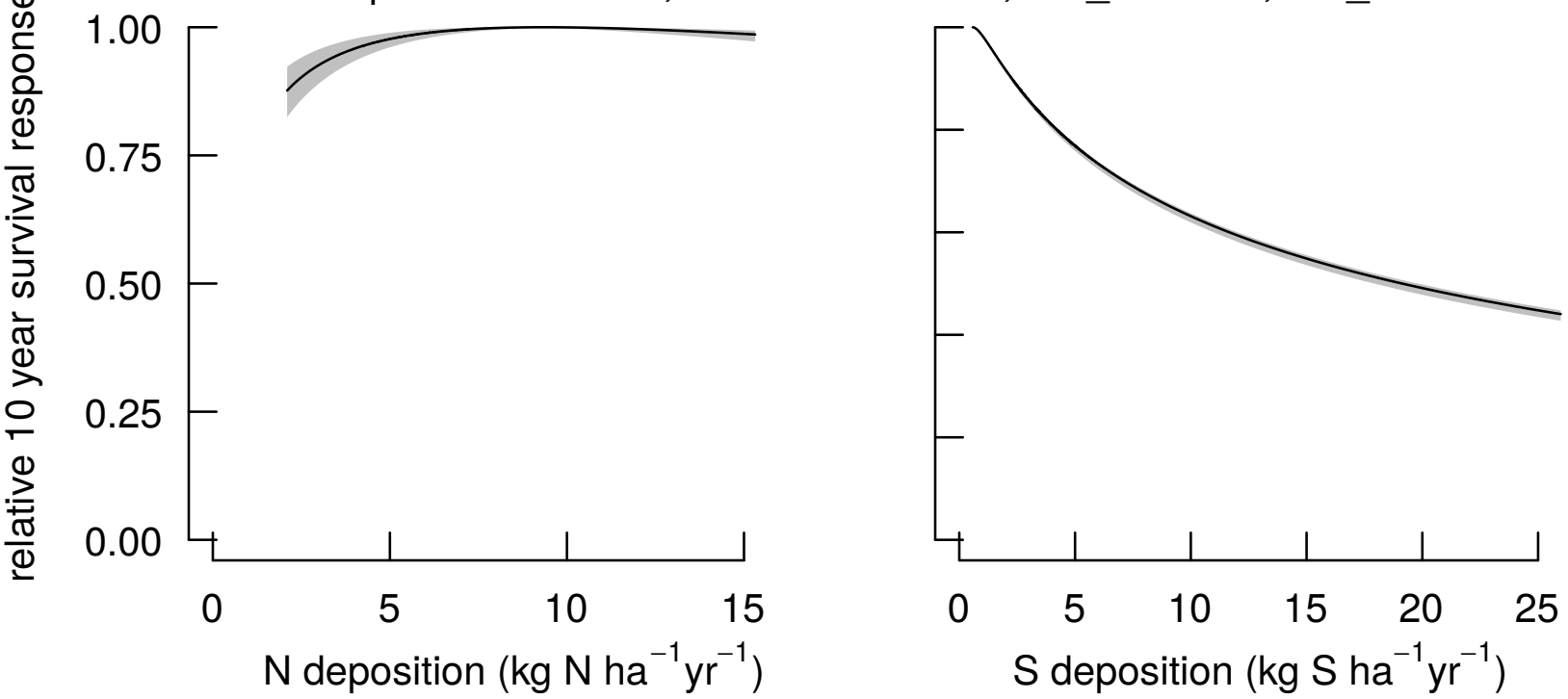
American hornbeam, musclewood

Carpinus caroliniana

N deposition

S deposition
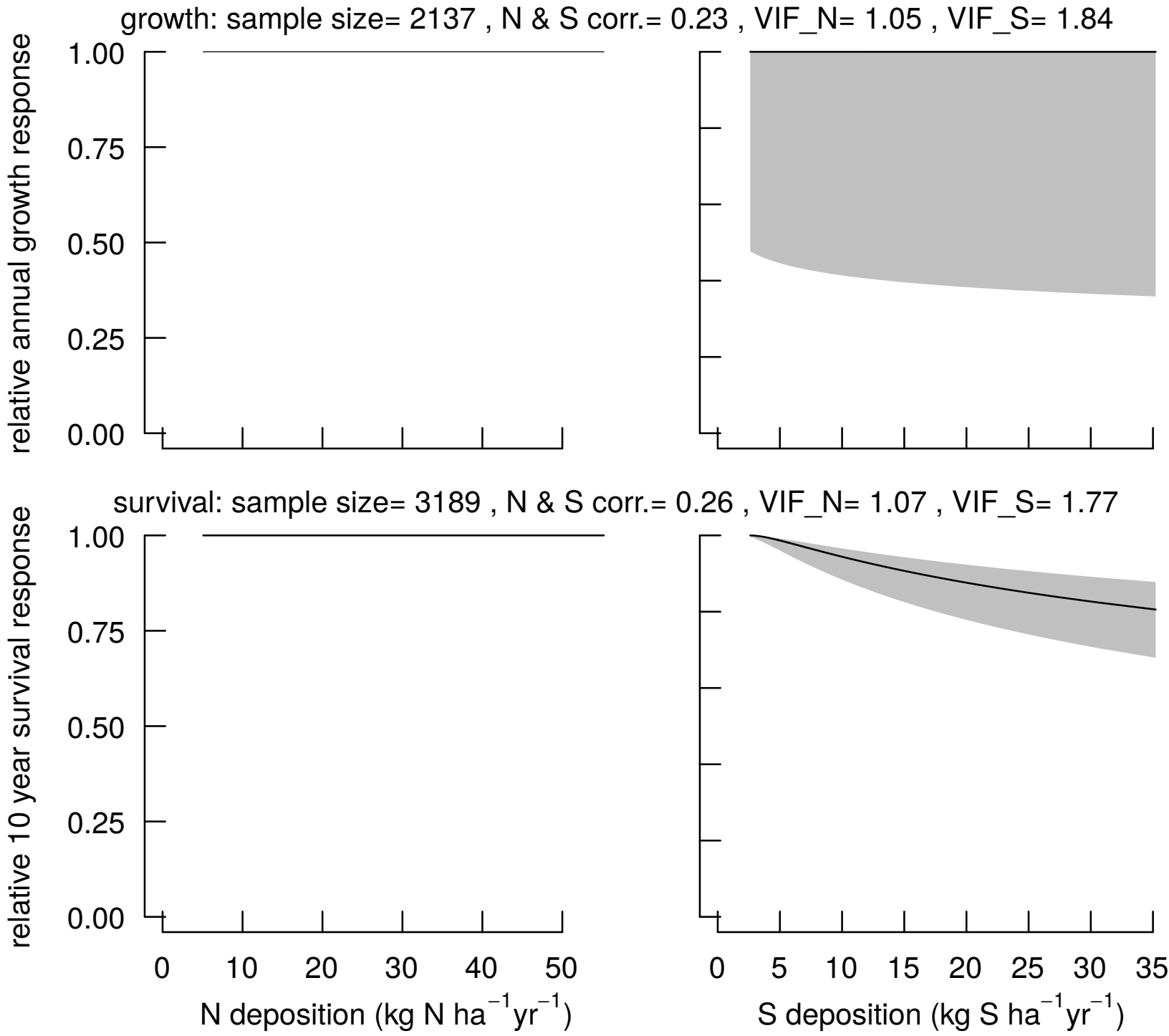


\section{bitternut hickory}

N deposition

Carya cordiformis

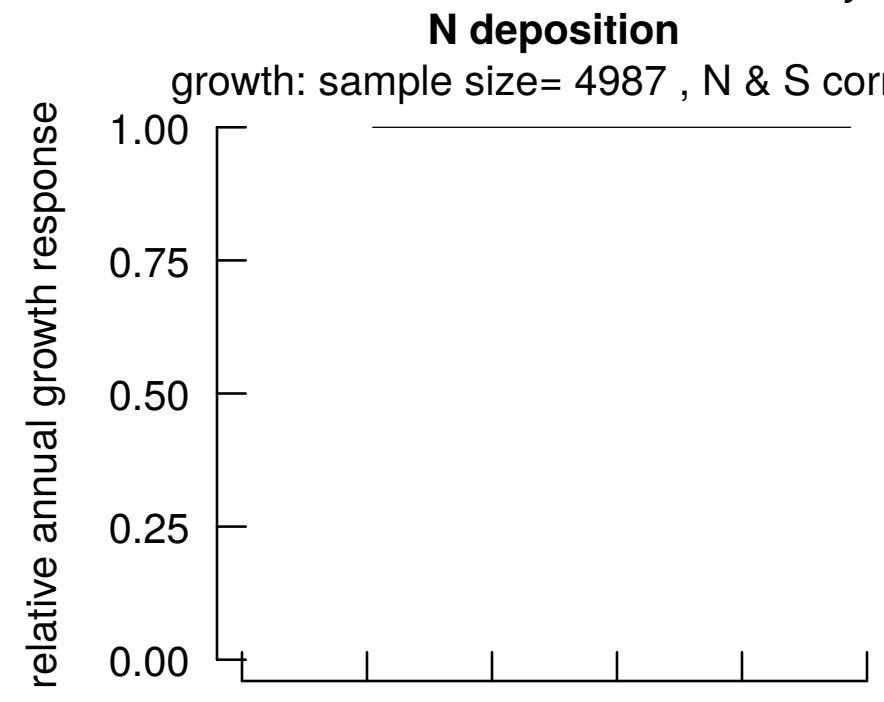

$S$ deposition
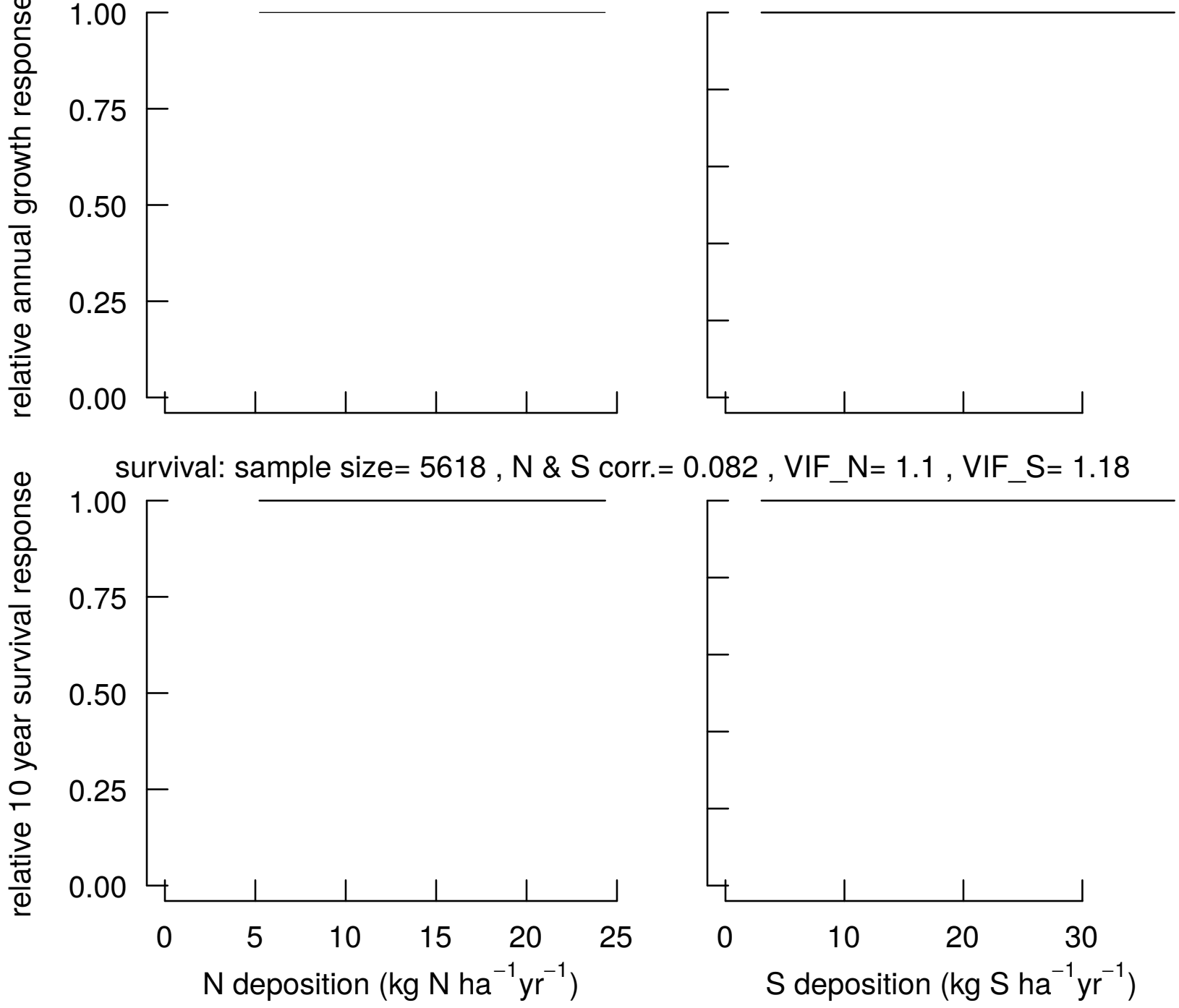


\section{pignut hickory}

$\mathrm{N}$ deposition

Carya glabra

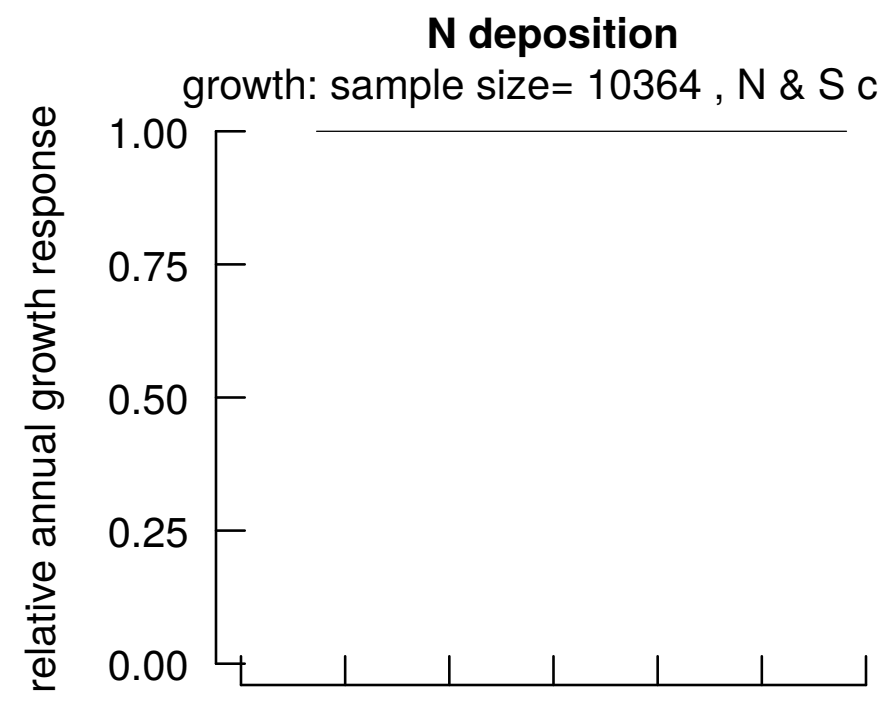

$S$ deposition
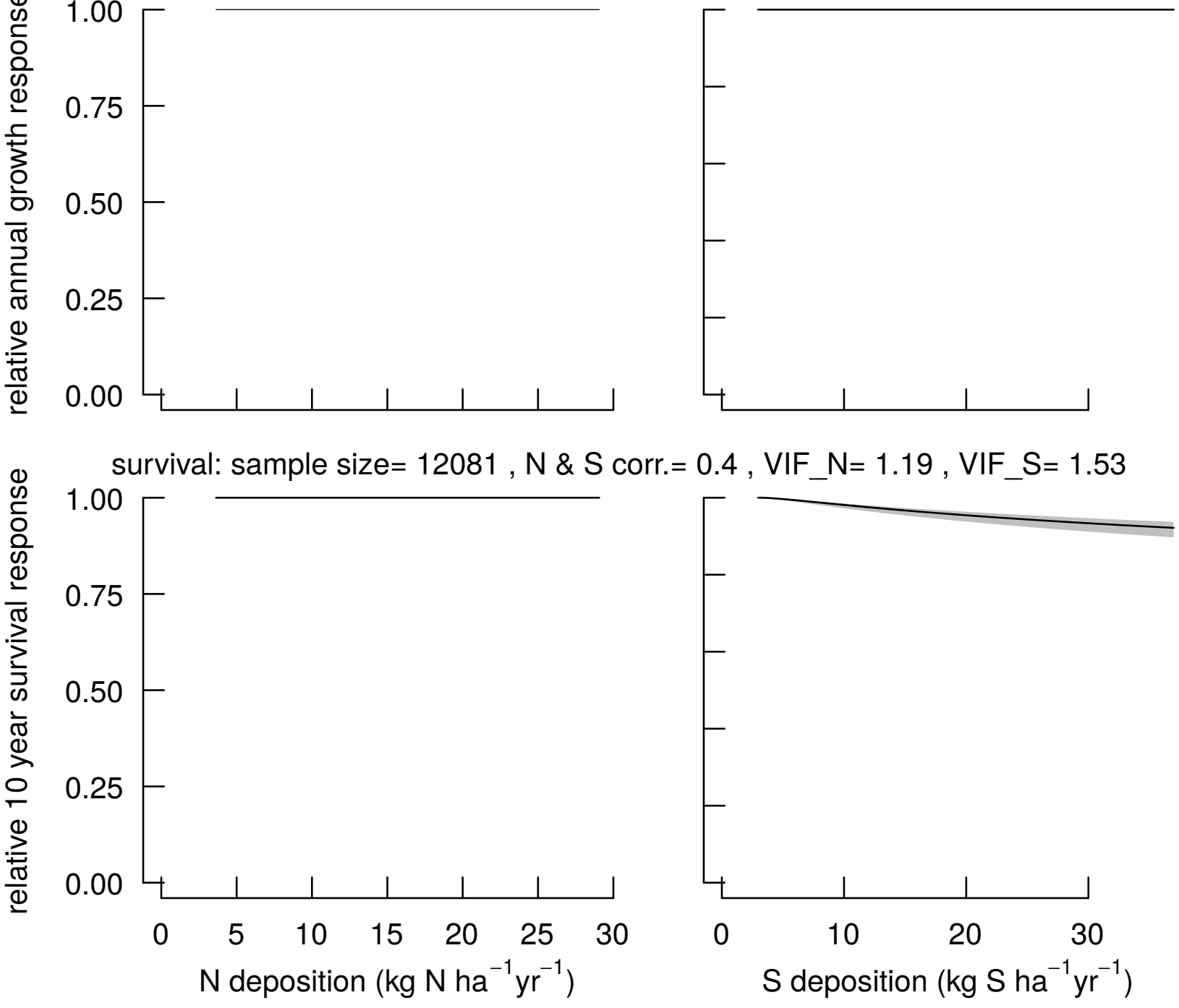


\section{shagbark hickory}

N deposition

Carya ovata

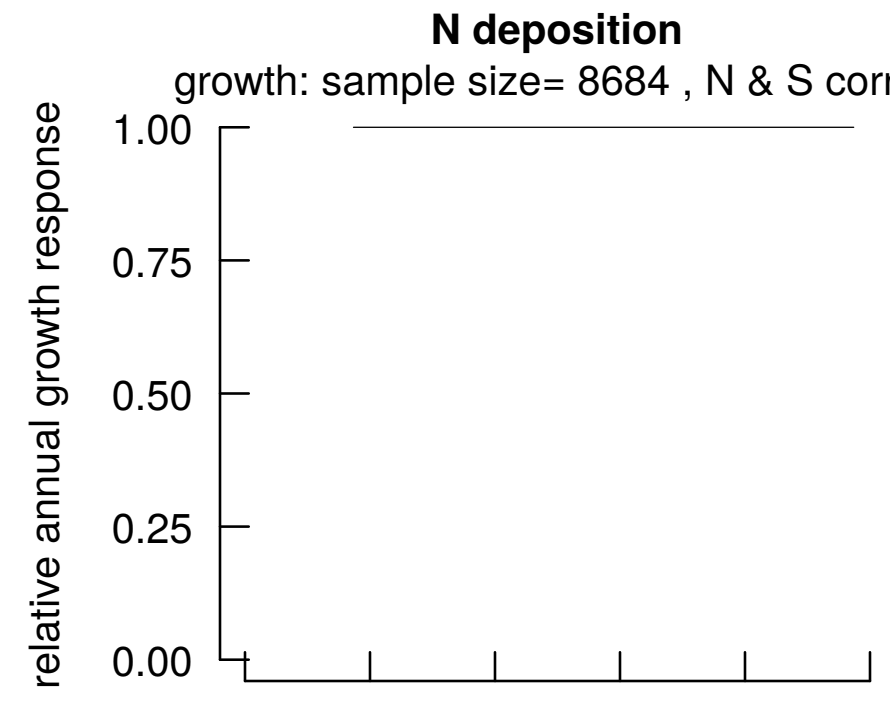

\section{$S$ deposition}
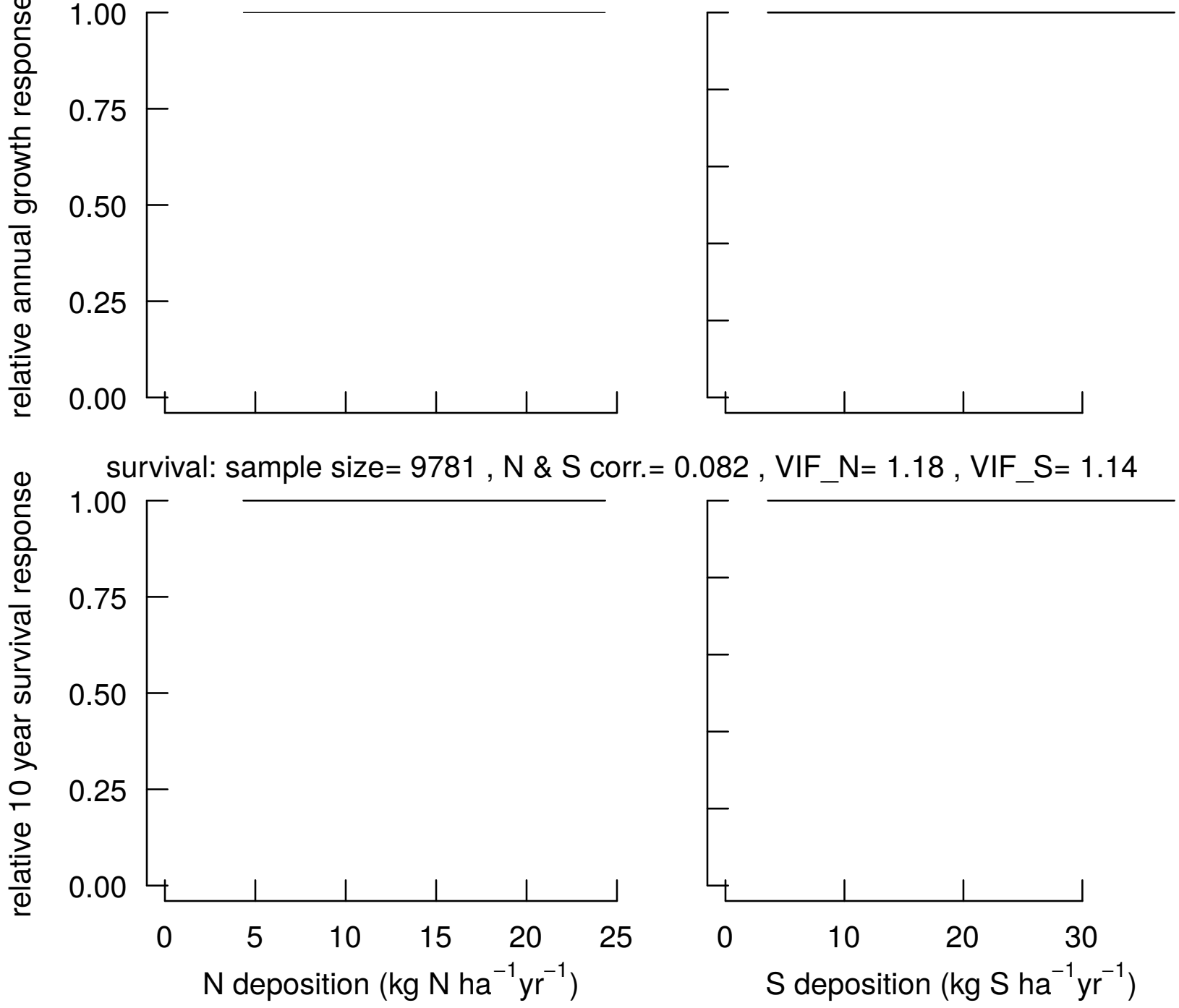


\section{black hickory}

$\mathbf{N}$ deposition

Carya texana

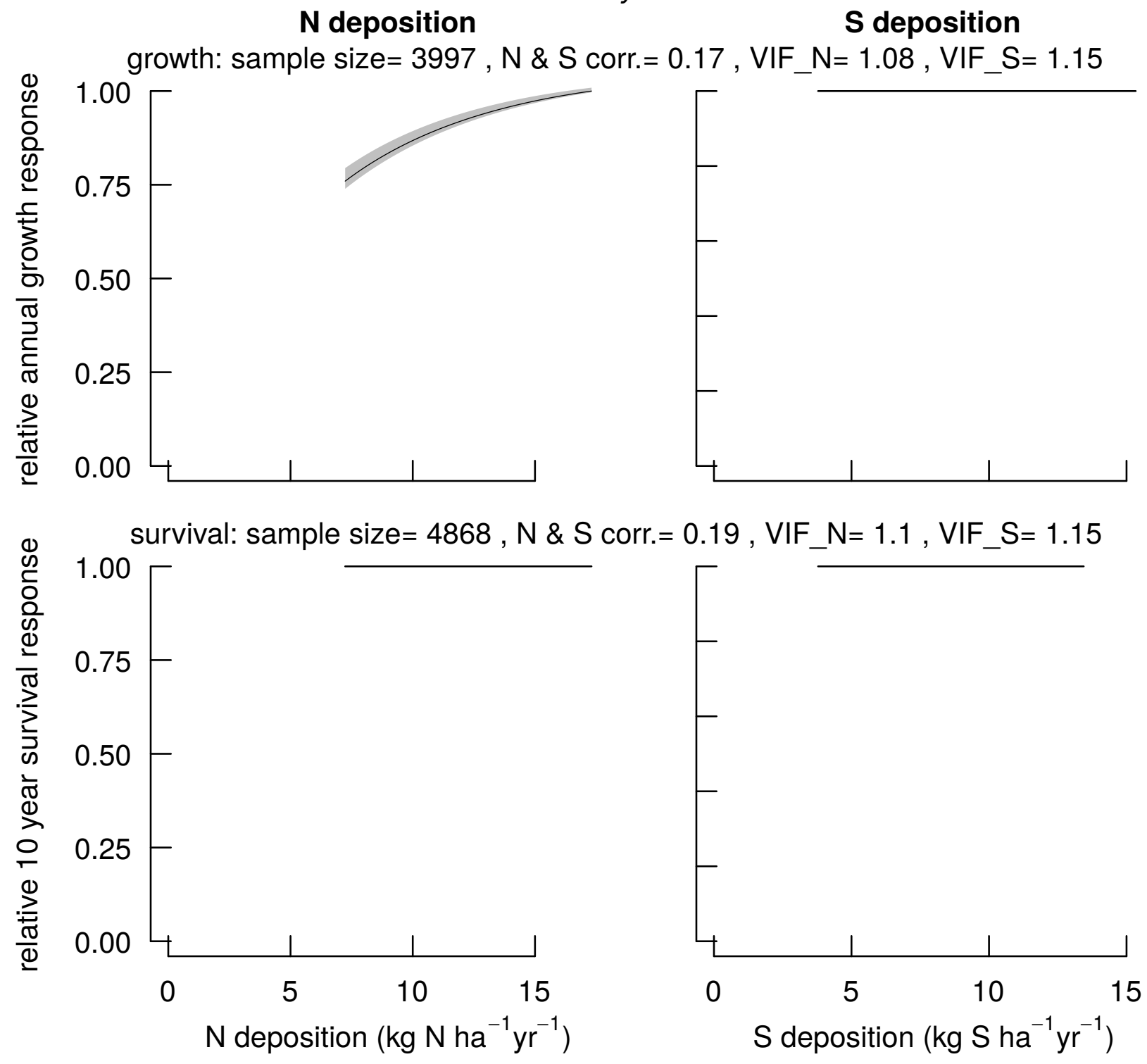




\section{mockernut hickory}

$\mathbf{N}$ deposition

Carya alba

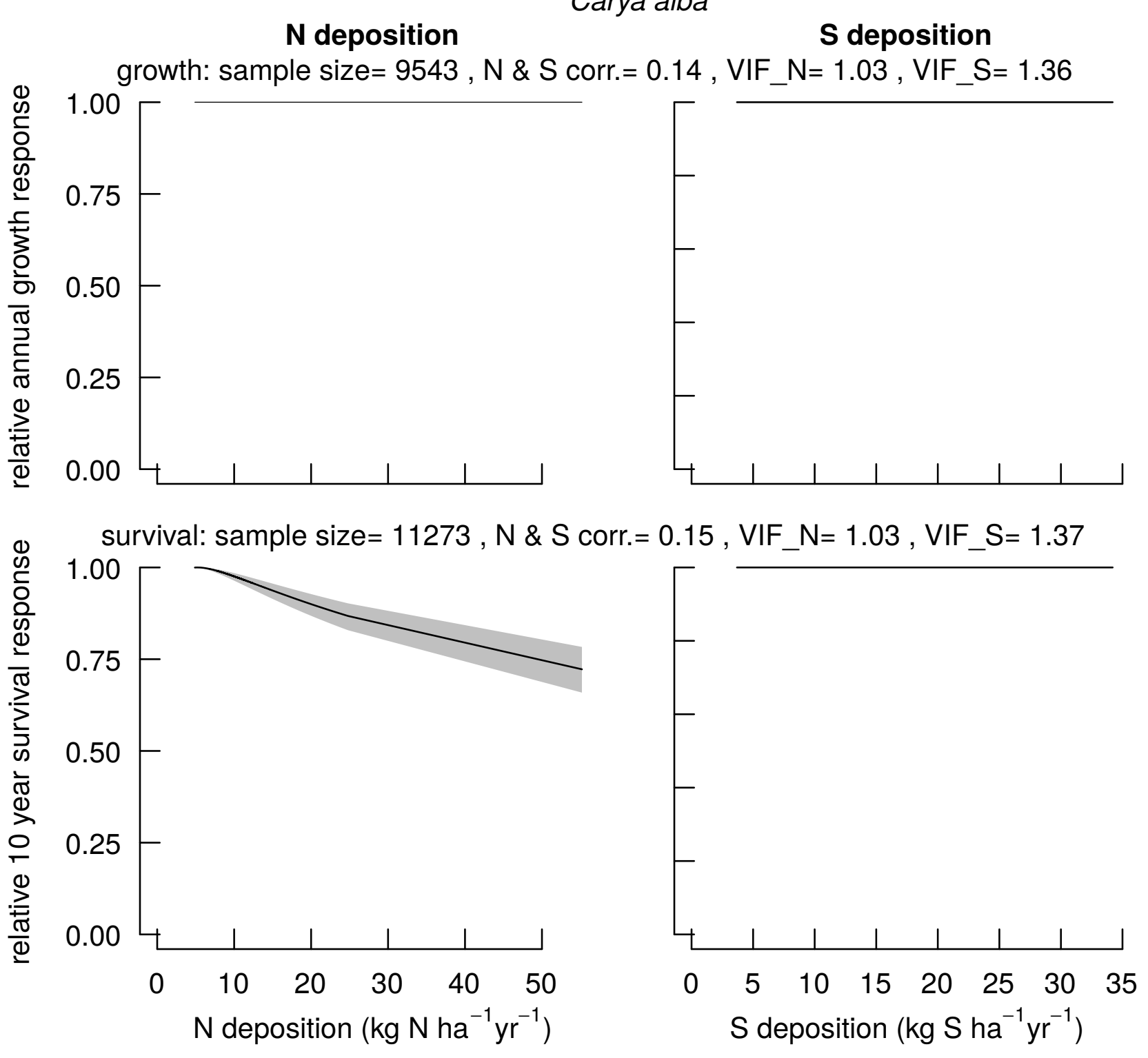




\section{sugarberry}

N deposition

Celtis laevigata

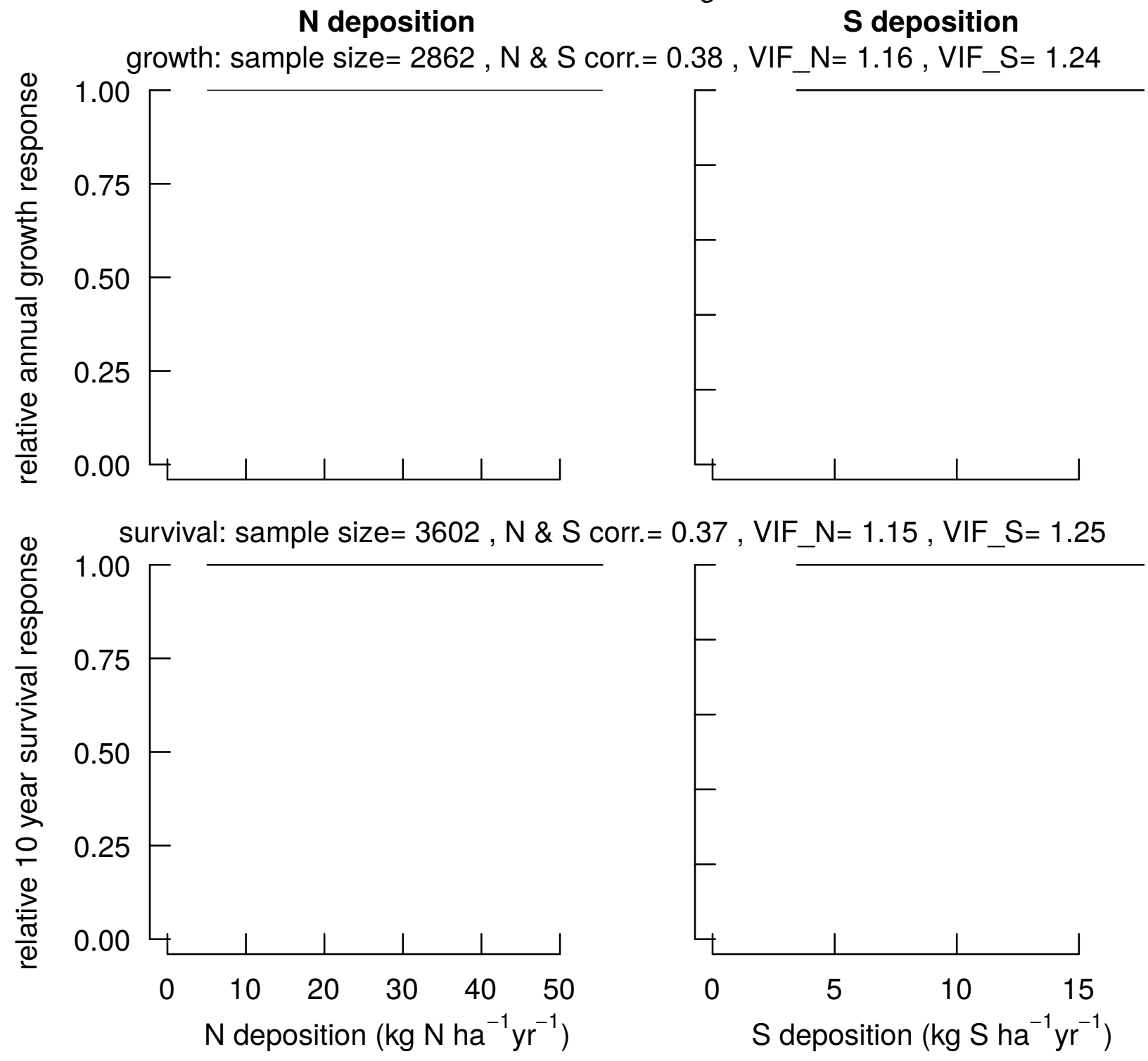


Celtis occidentalis

\section{N deposition}

S deposition

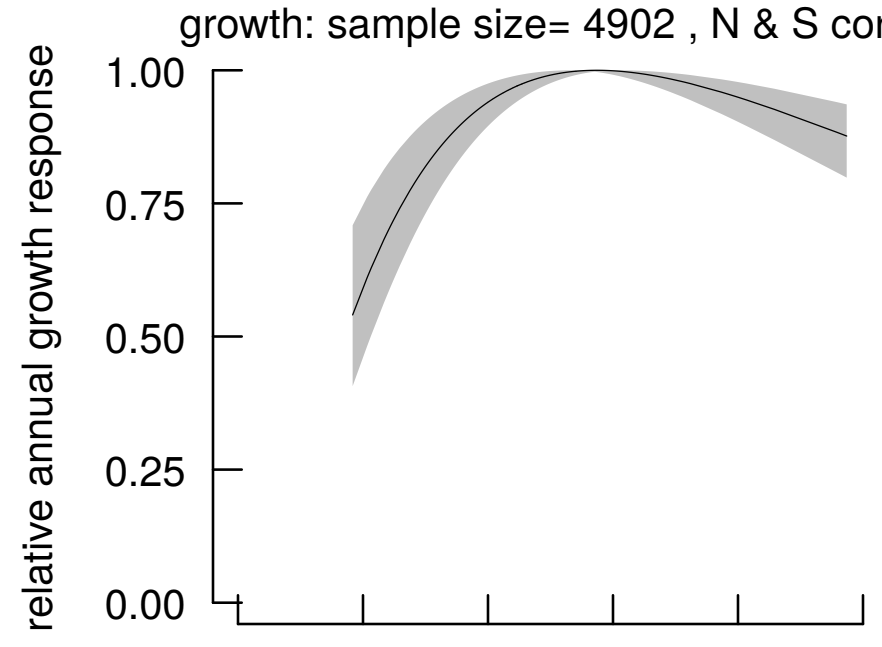

0.17, VIF_N=1.45,VIF_S= 1.58
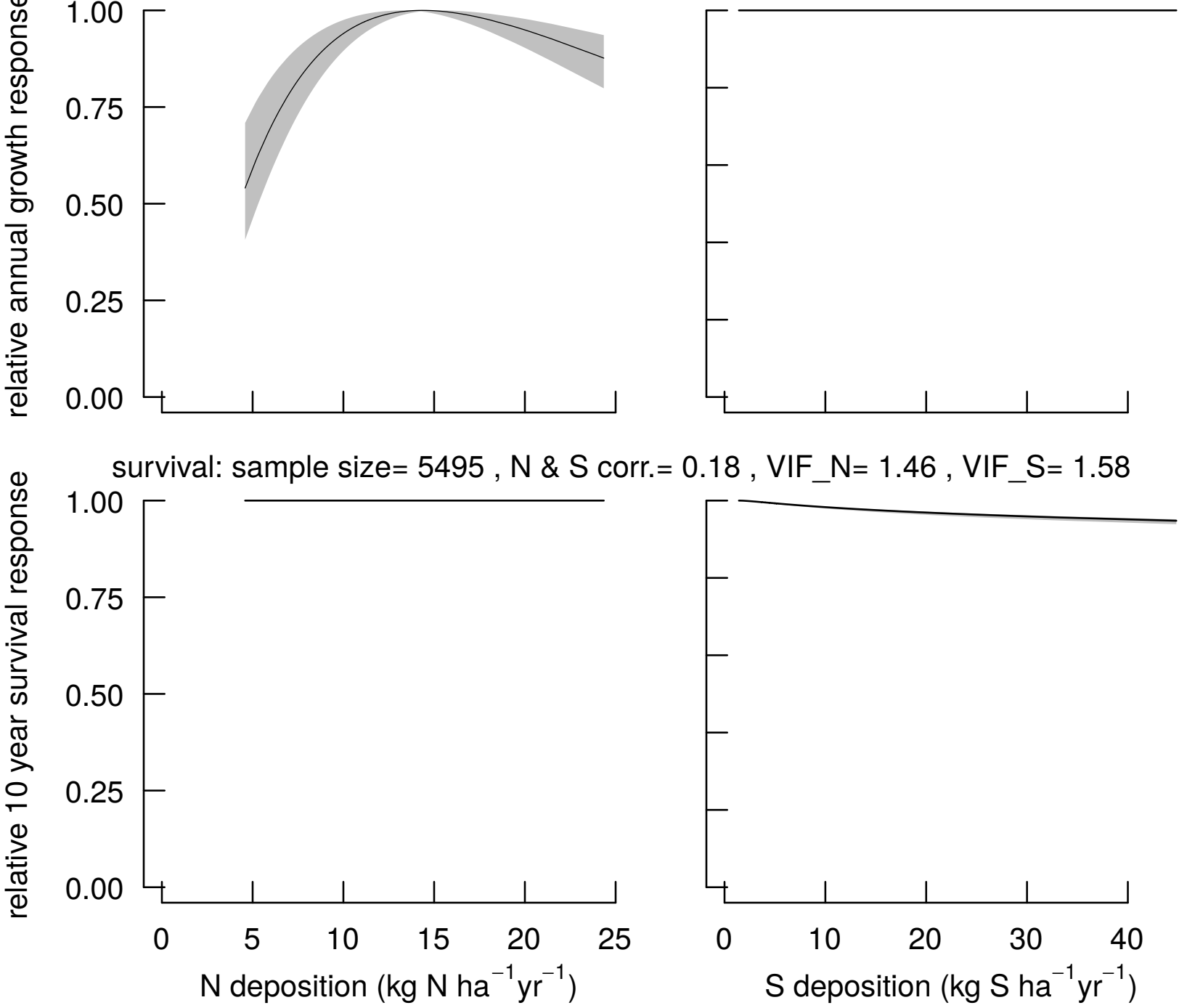

$0.18, V I F \_N=1.46, V I F \_S=1.58$ 


\section{American beech}

N deposition

Fagus grandifolia

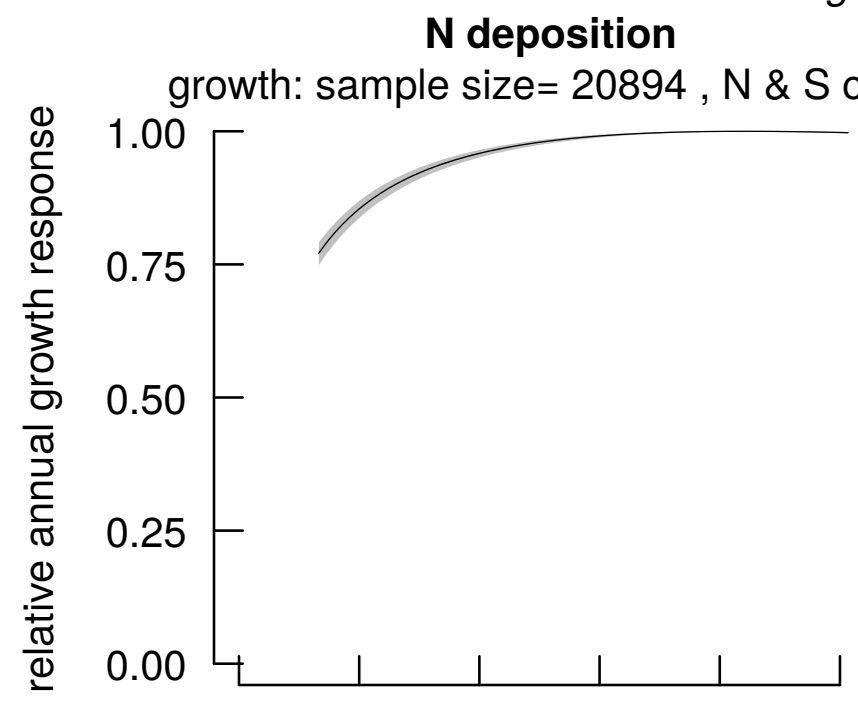

$S$ deposition
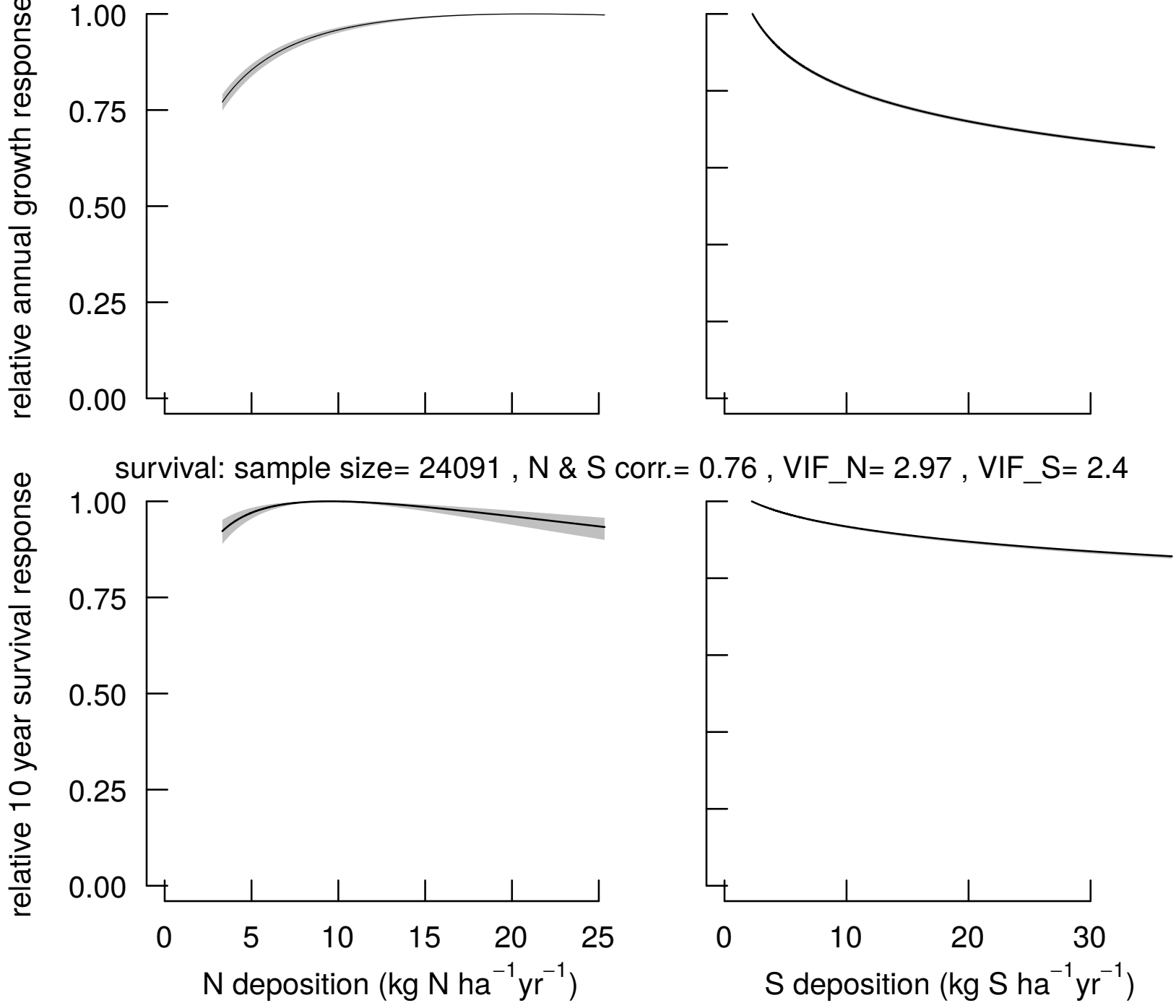


\section{white ash}

Fraxinus americana

\section{N deposition}

$S$ deposition
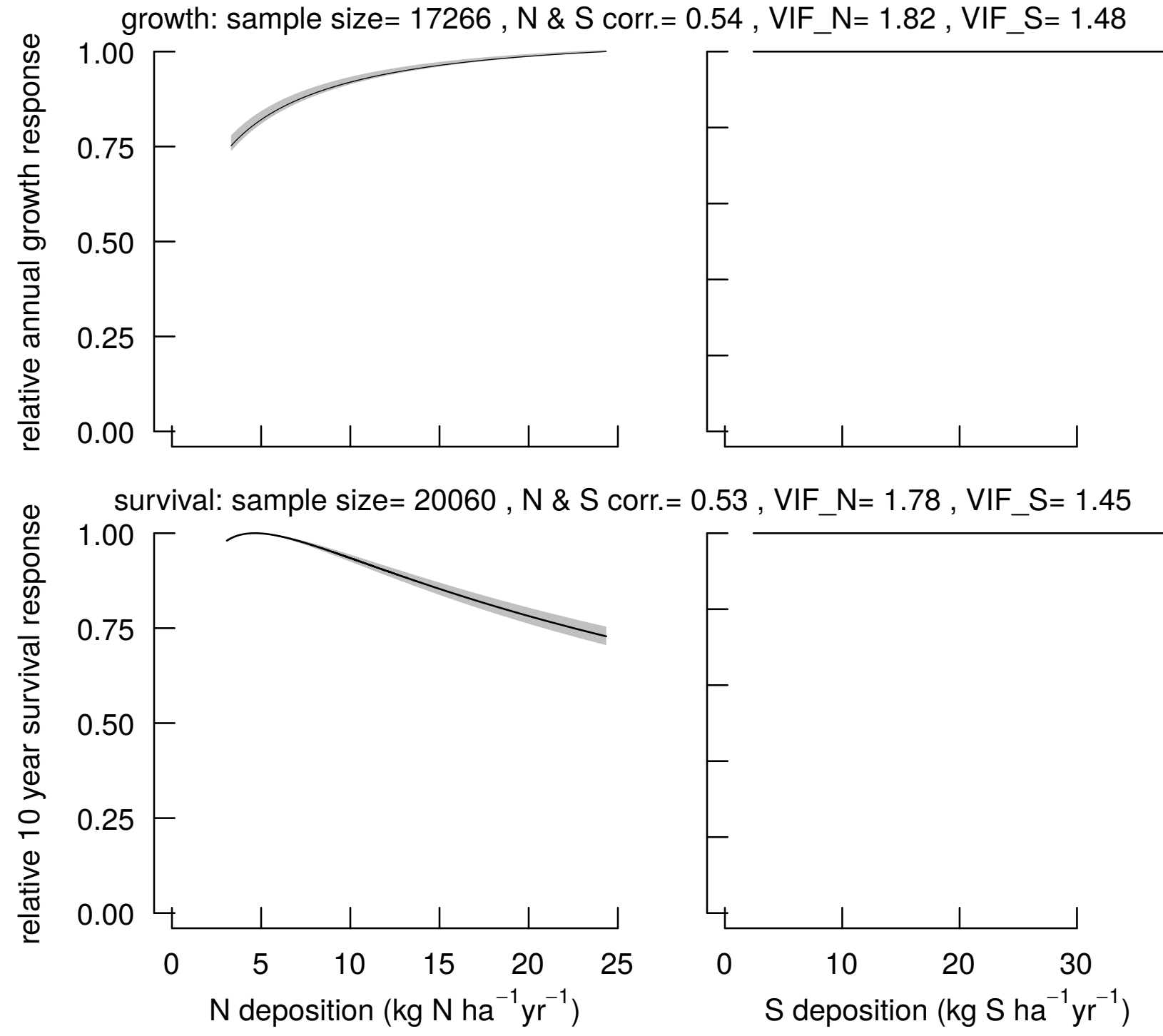


\section{black ash \\ Fraxinus nigra}

N deposition
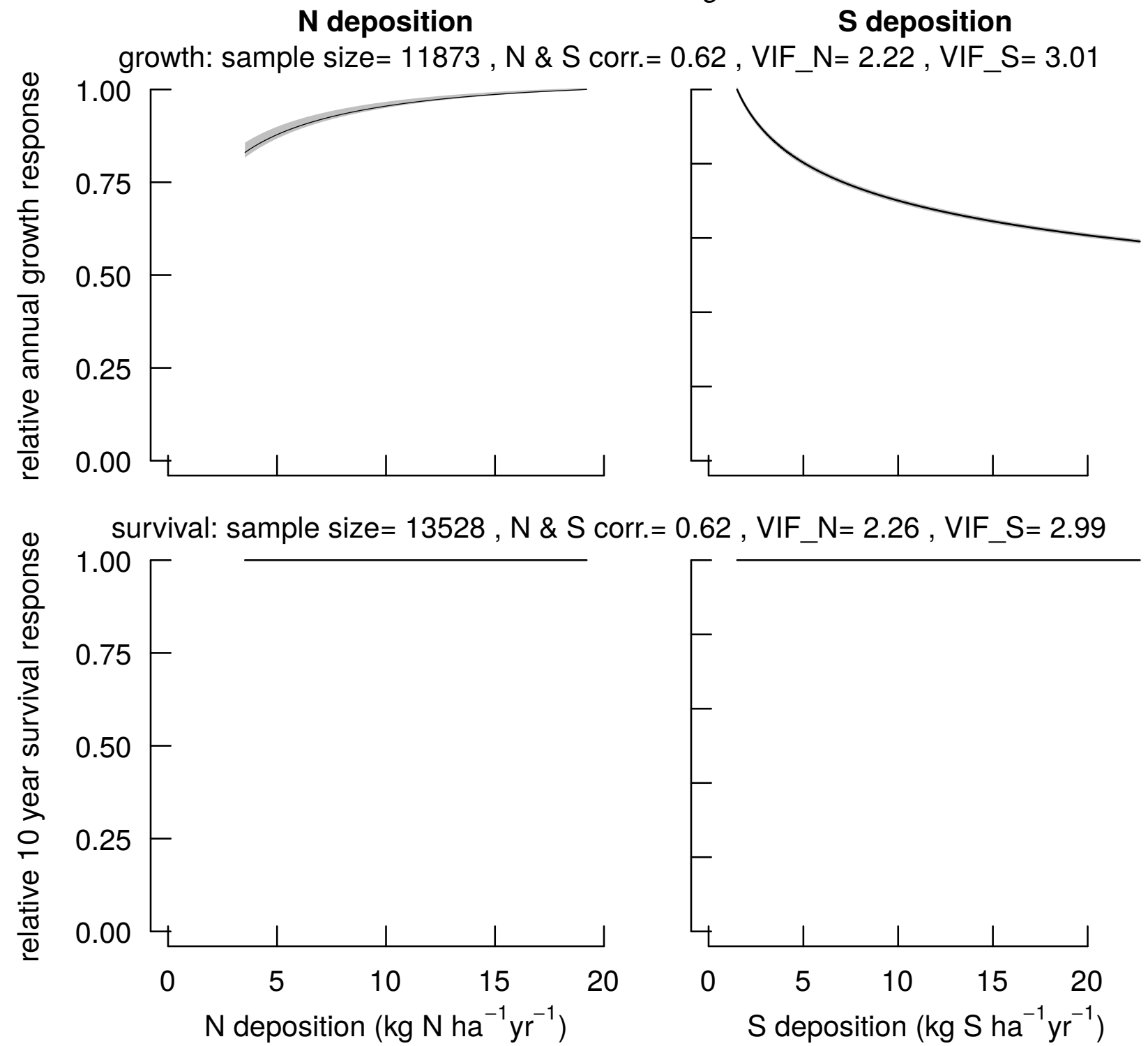


\section{green ash \\ Fraxinus pennsylvanica \\ N deposition \\ $S$ deposition}
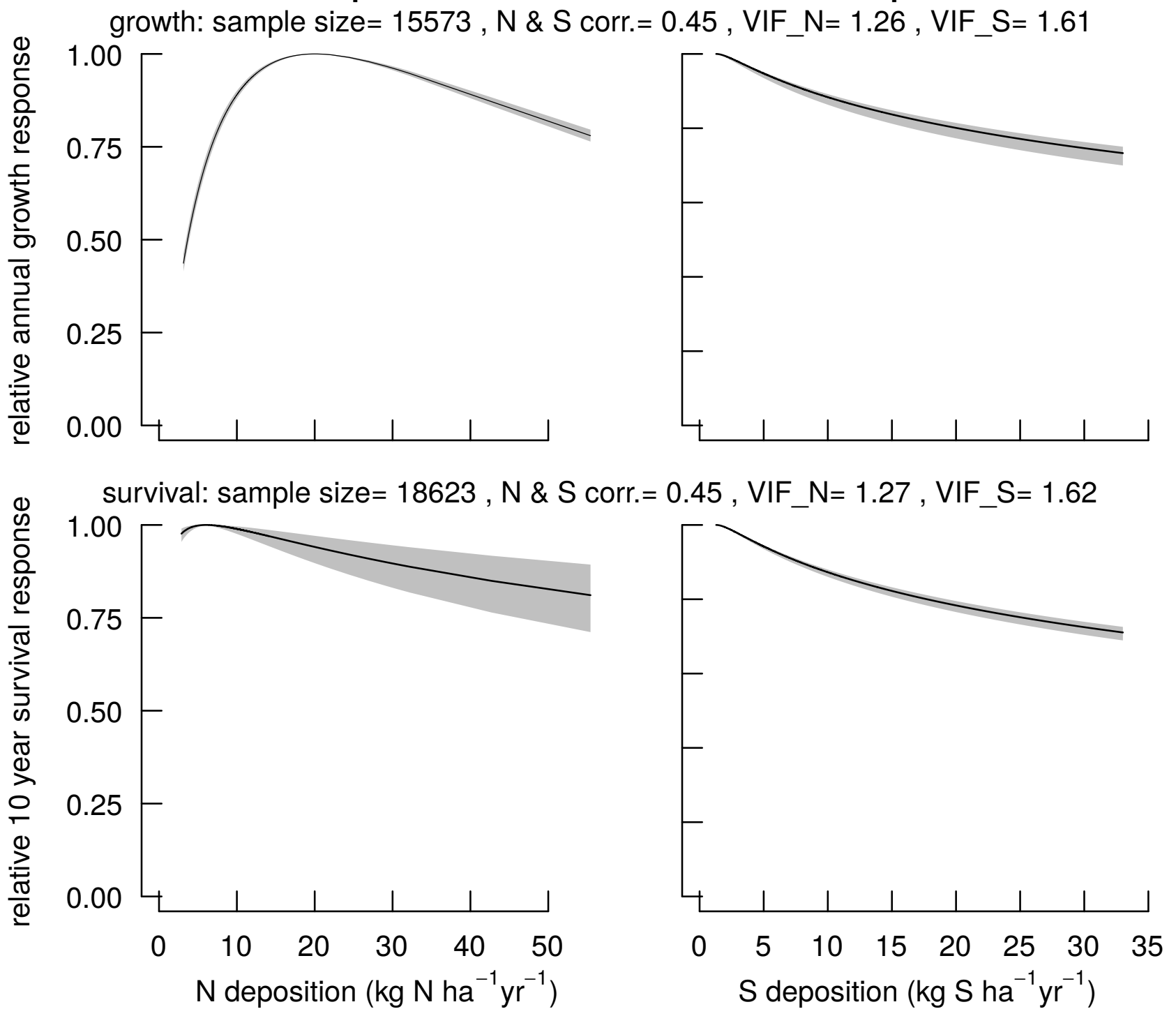


\section{honeylocust}

Gleditsia triacanthos

\section{$\mathbf{N}$ deposition}

\section{$\mathrm{S}$ deposition}

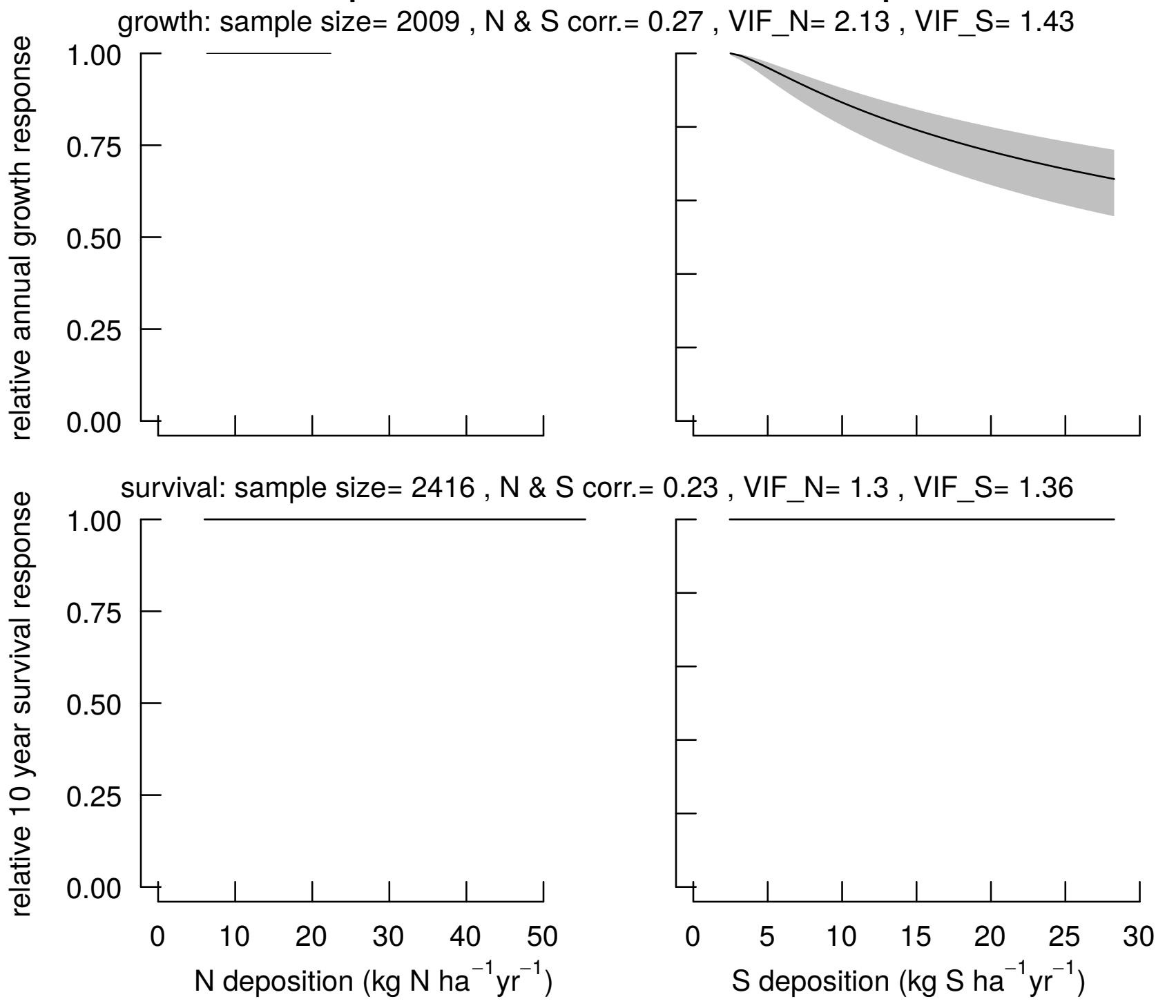




\section{black walnut \\ Juglans nigra}

N deposition
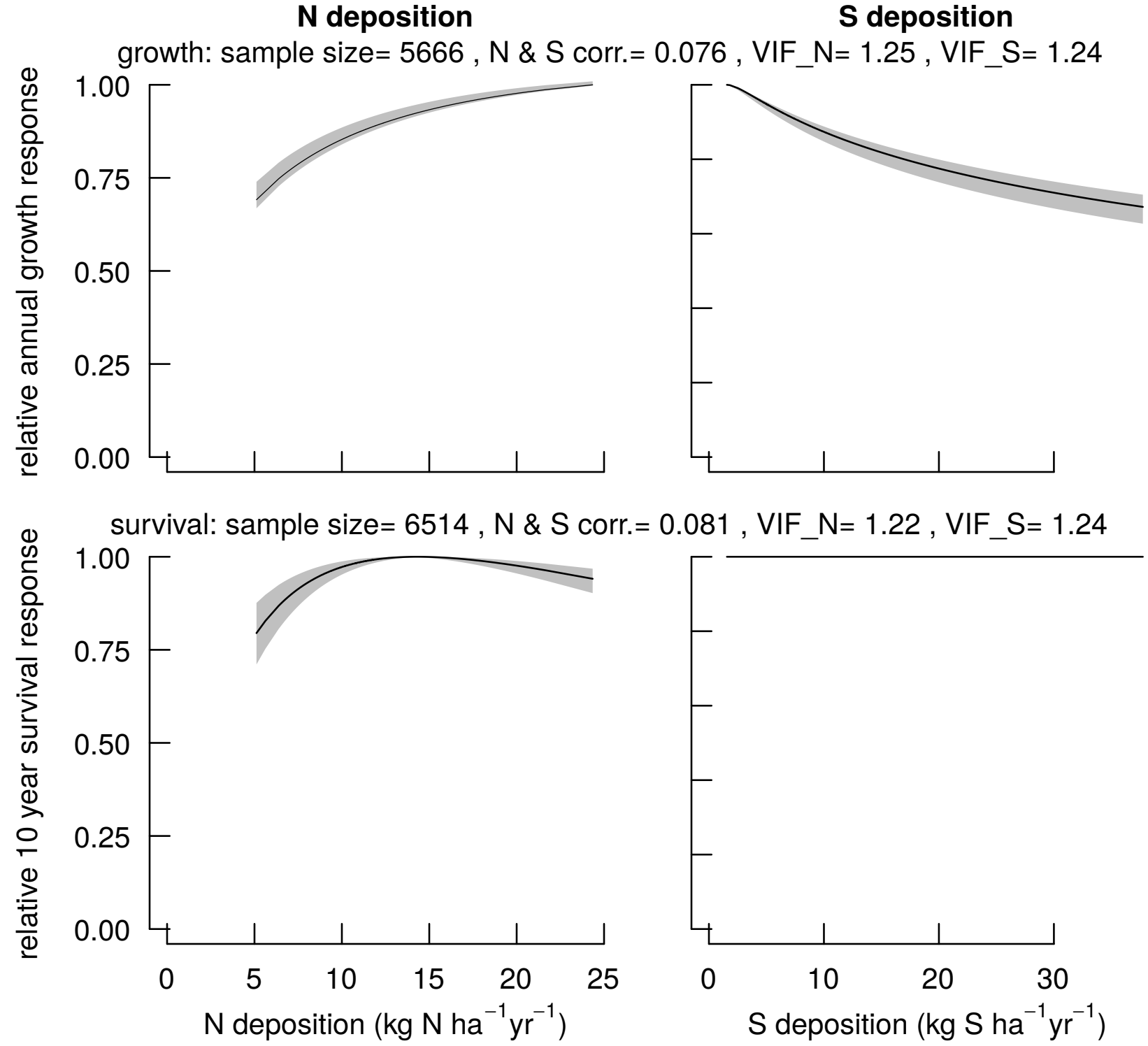


\section{sweetgum \\ $\mathrm{N}$ deposition \\ Liquidambar styraciflua}
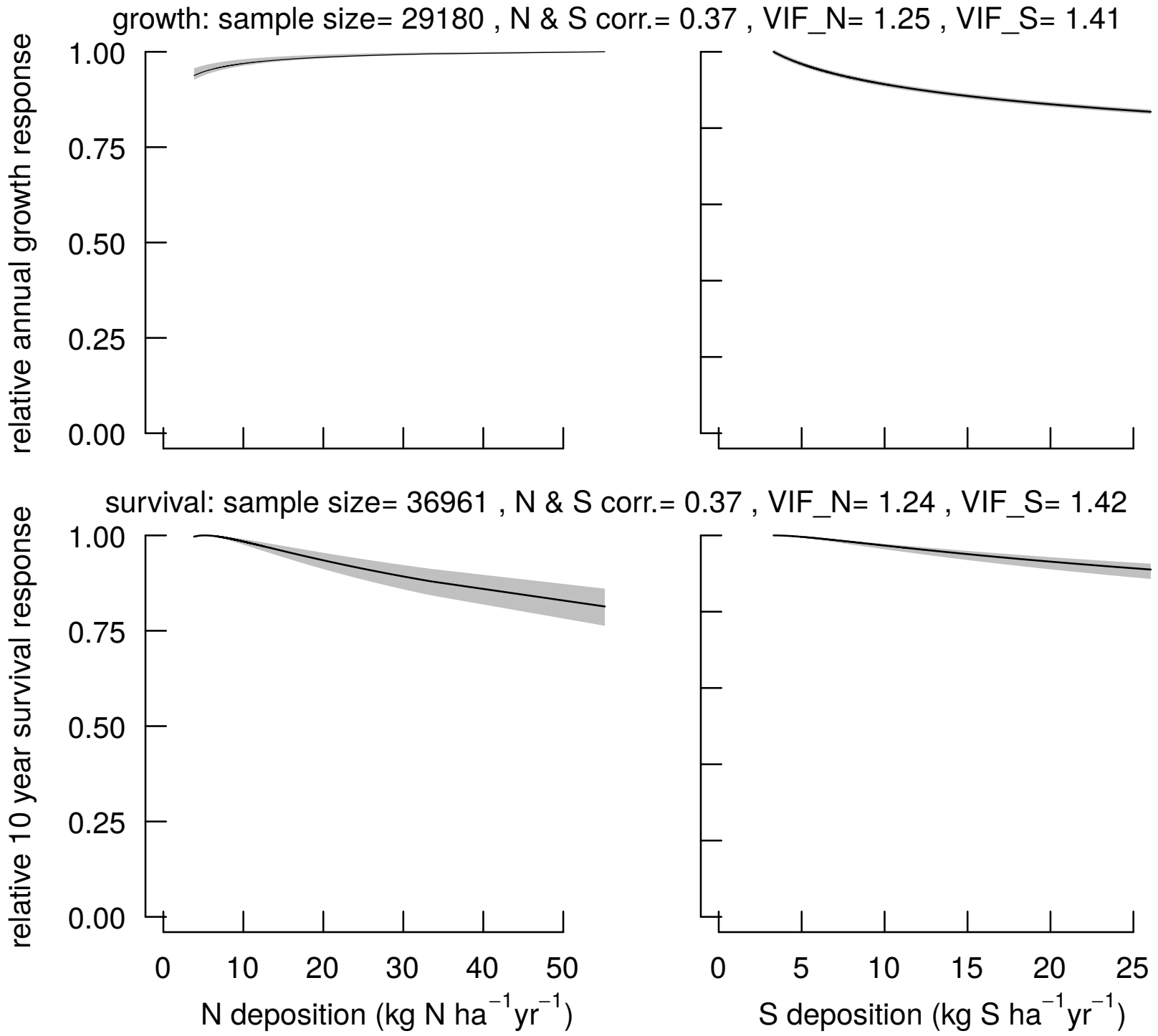


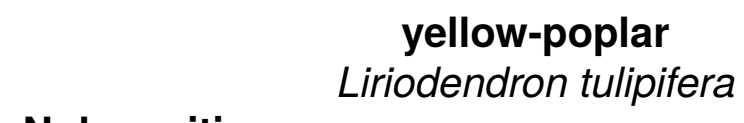

$\mathrm{N}$ deposition

Liriodendron tulipifera

$\mathrm{S}$ deposition

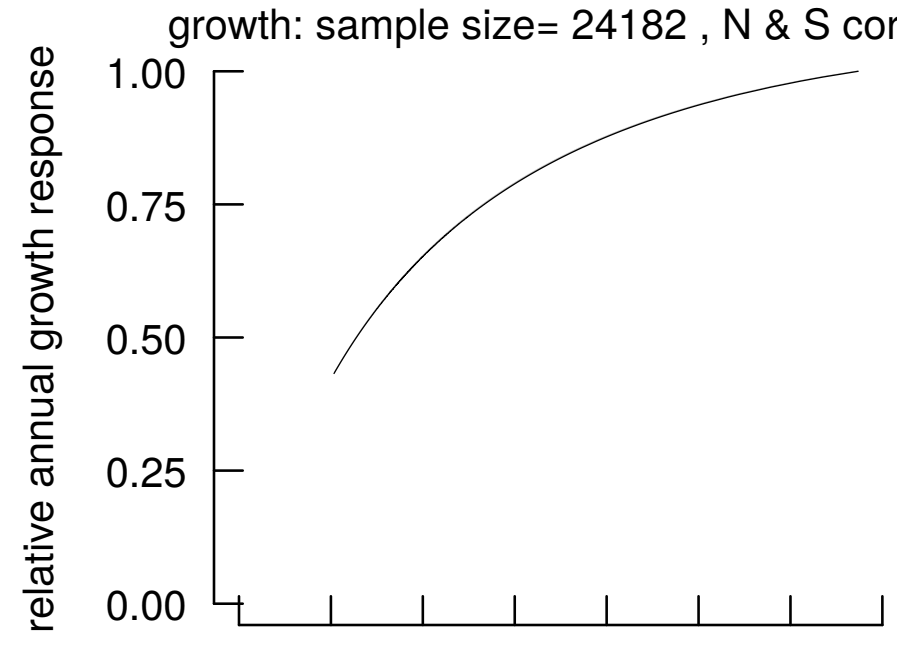

0.41, VIF_N $=1.21$, VIF_S $=1.93$
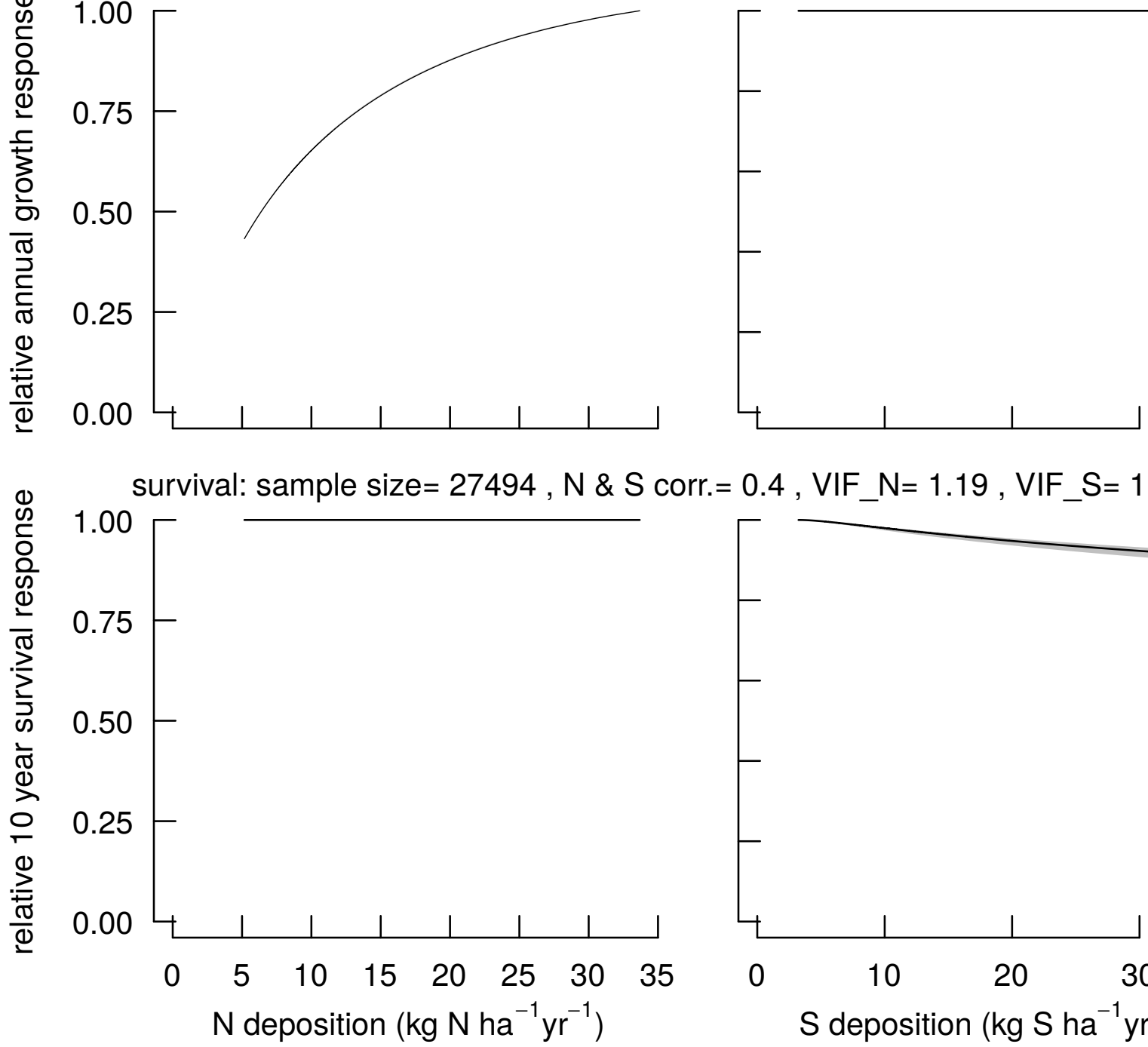

orr. $=0.4$, VIF_N=1.19, VIF_S $=1.9$ 


\section{tanoak}

$\mathbf{N}$ deposition

Lithocarpus densiflorus

\section{$\mathrm{S}$ deposition}
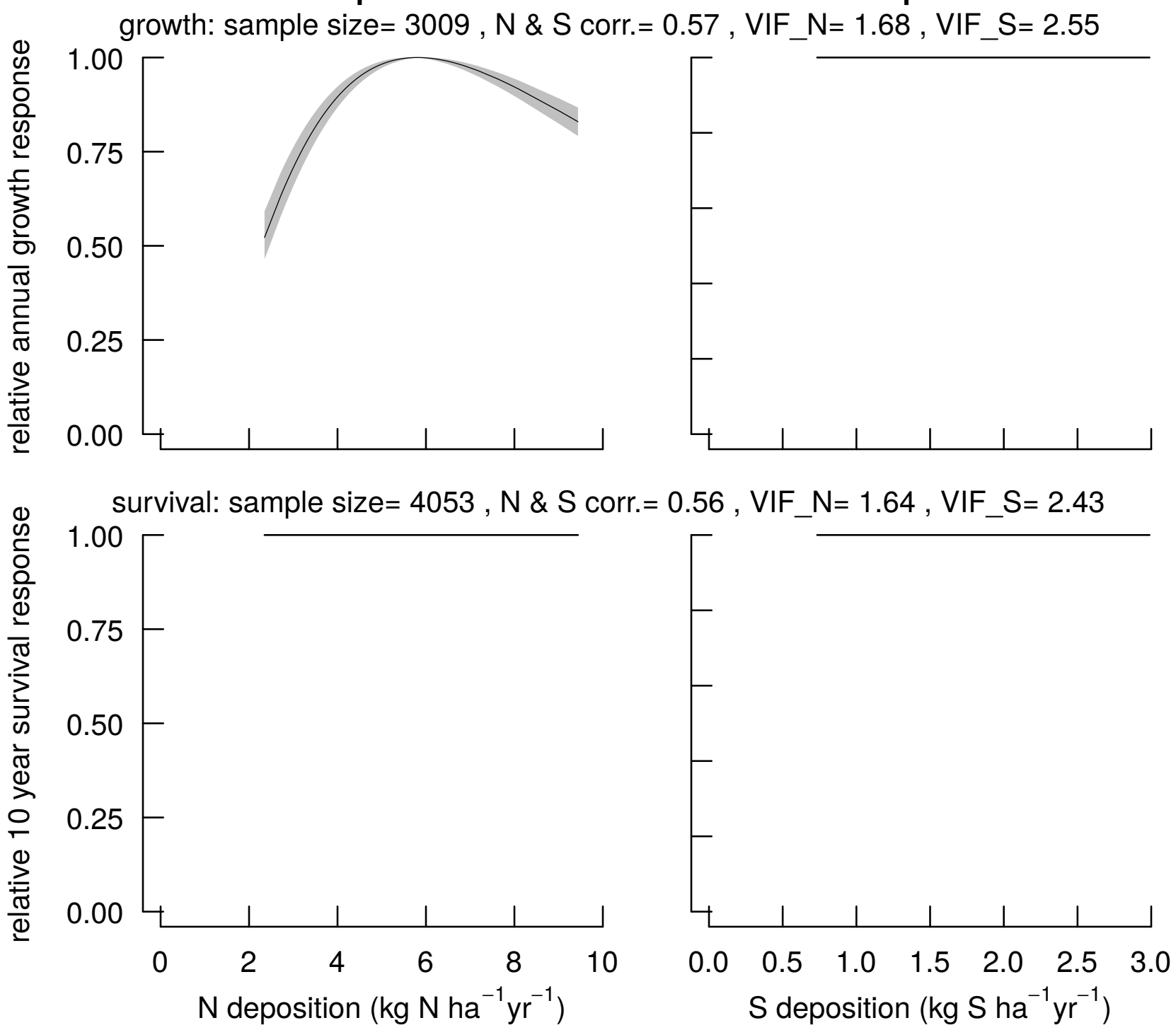


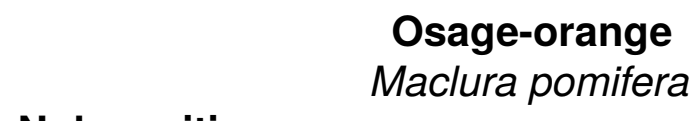

$\mathbf{N}$ deposition

Maclura pomifera
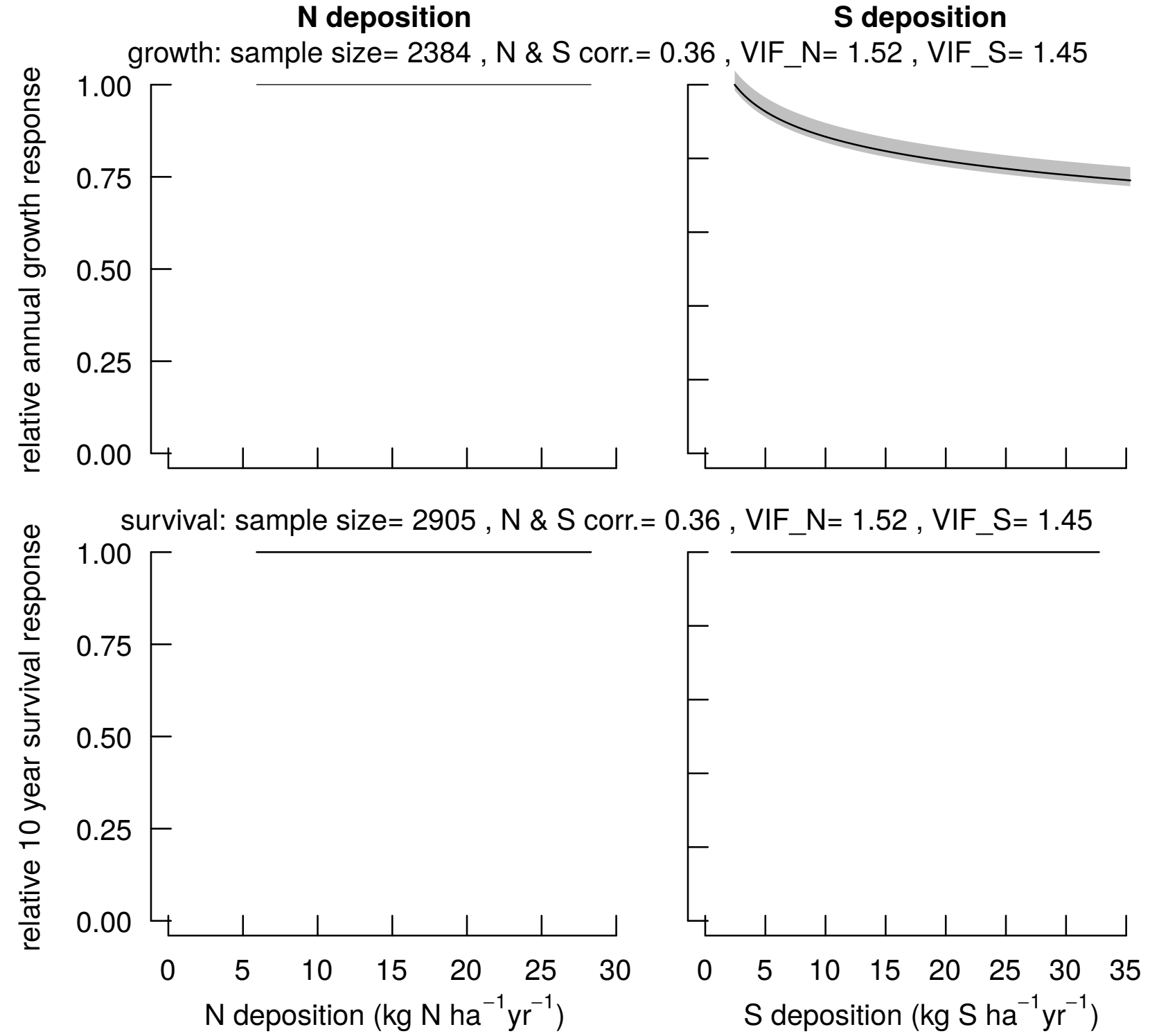


\section{sweetbay \\ Magnolia virginiana}

\section{N deposition \\ $\mathrm{S}$ deposition}
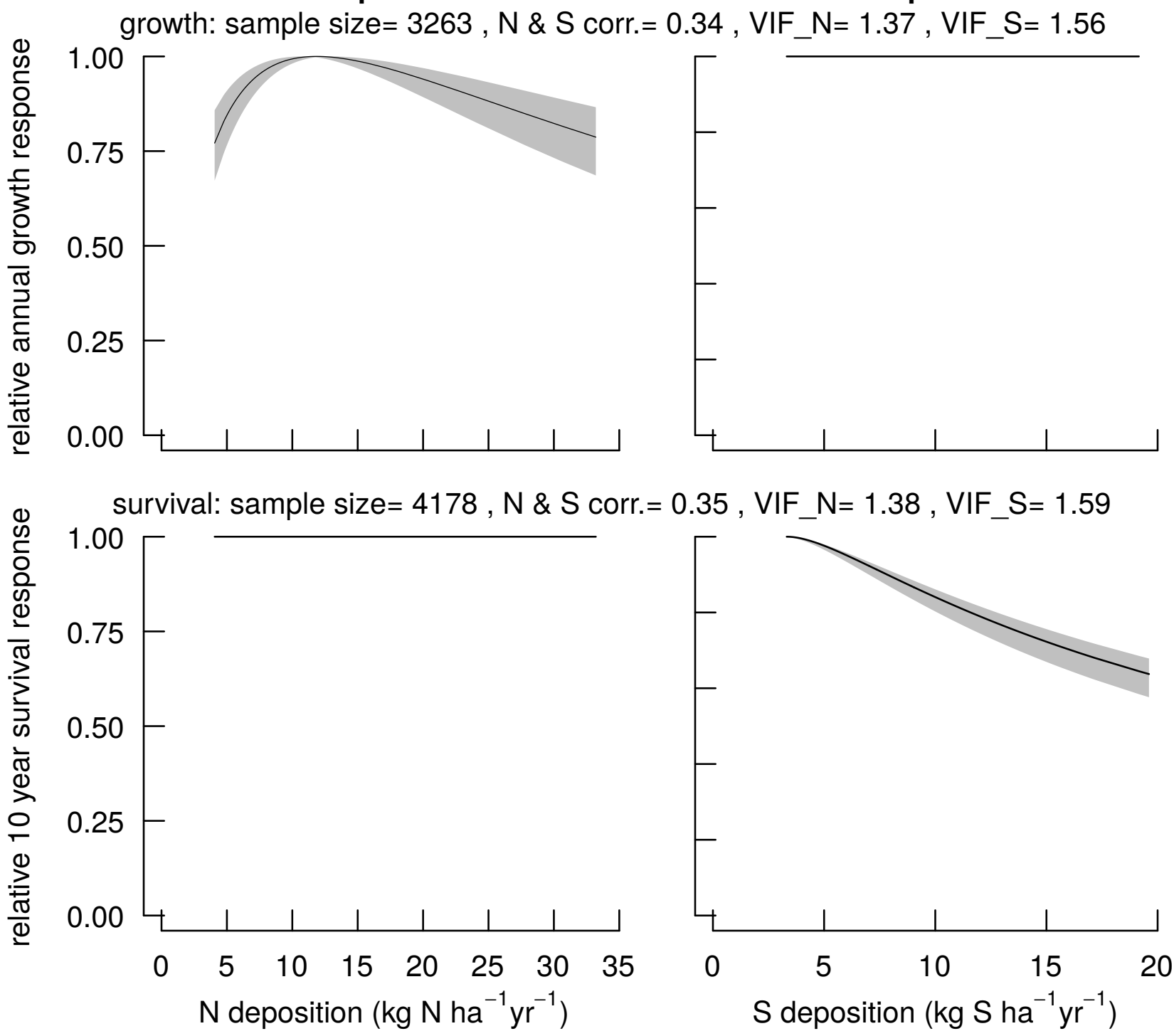


\section{water tupelo}

N deposition

Nyssa aquatica

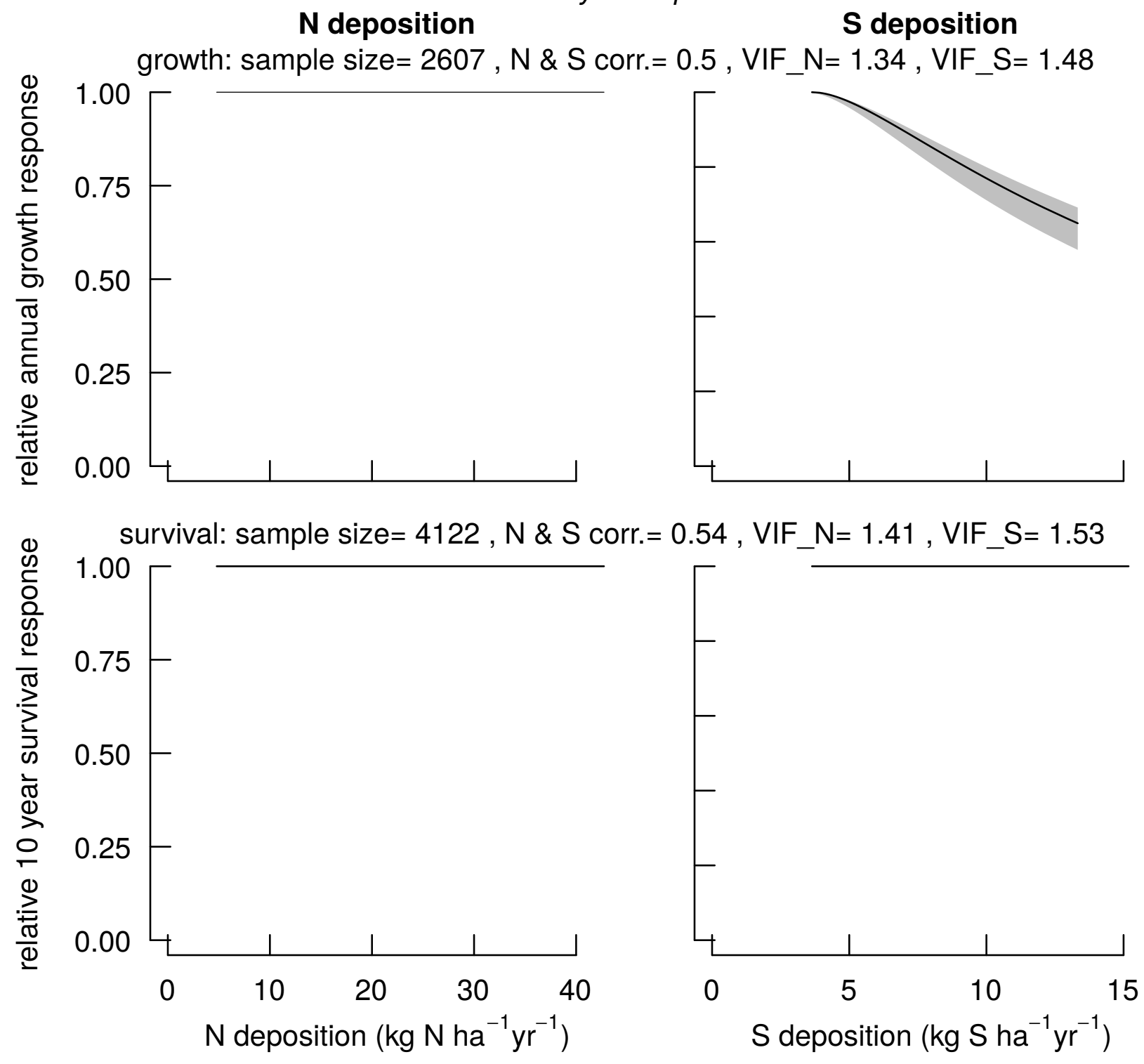




\section{blackgum}

$\mathrm{N}$ deposition

Nyssa sylvatica

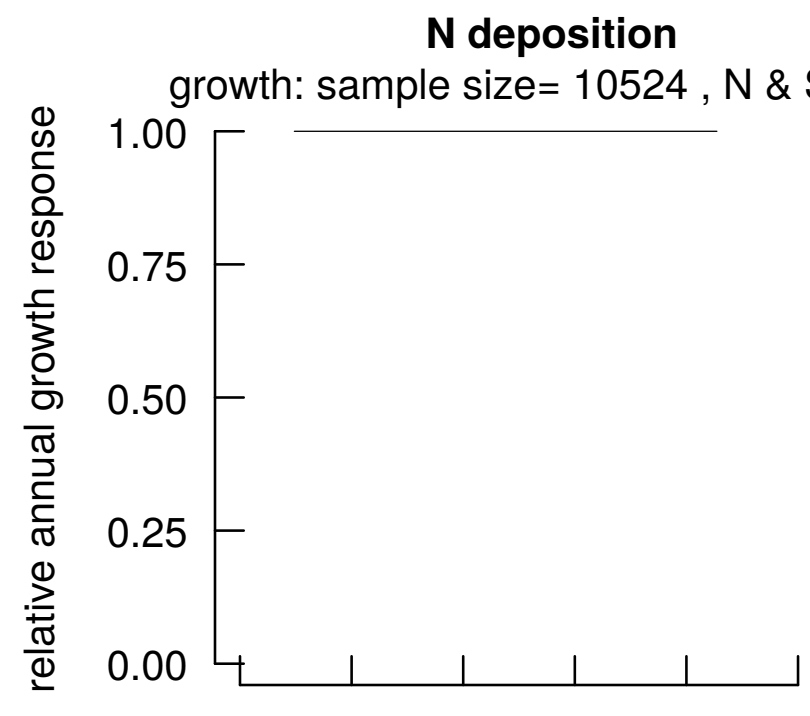

$S$ deposition

$S$ corr. $=0.43$, VIF_N $=1.28$, VIF_S $=1.85$

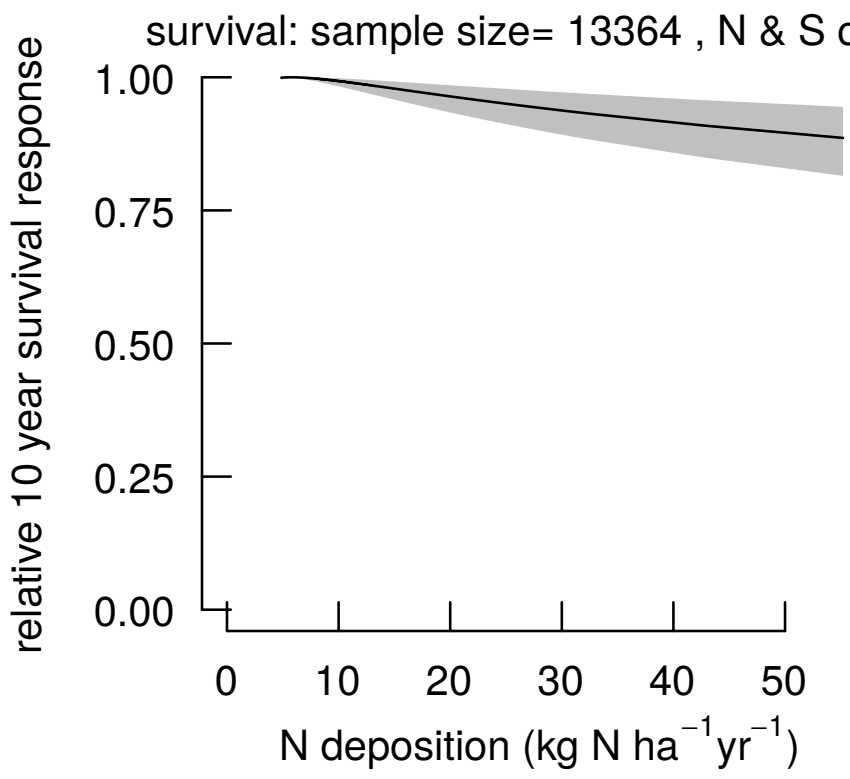

orr. $=0.42$, VIF_N=1.26, VIF_S $=1.83$ 

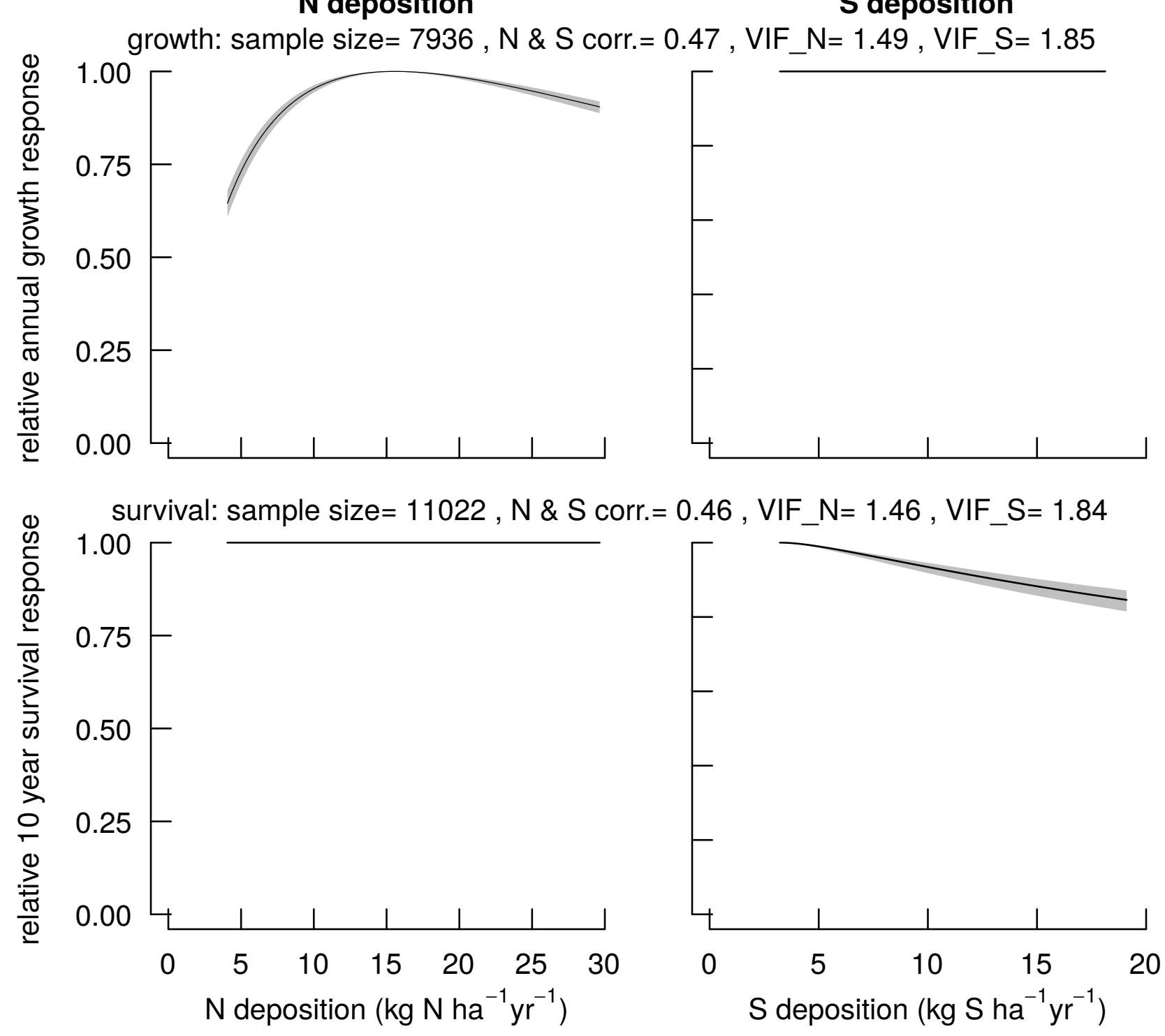


\section{eastern hophornbeam}

N deposition

Ostrya virginiana

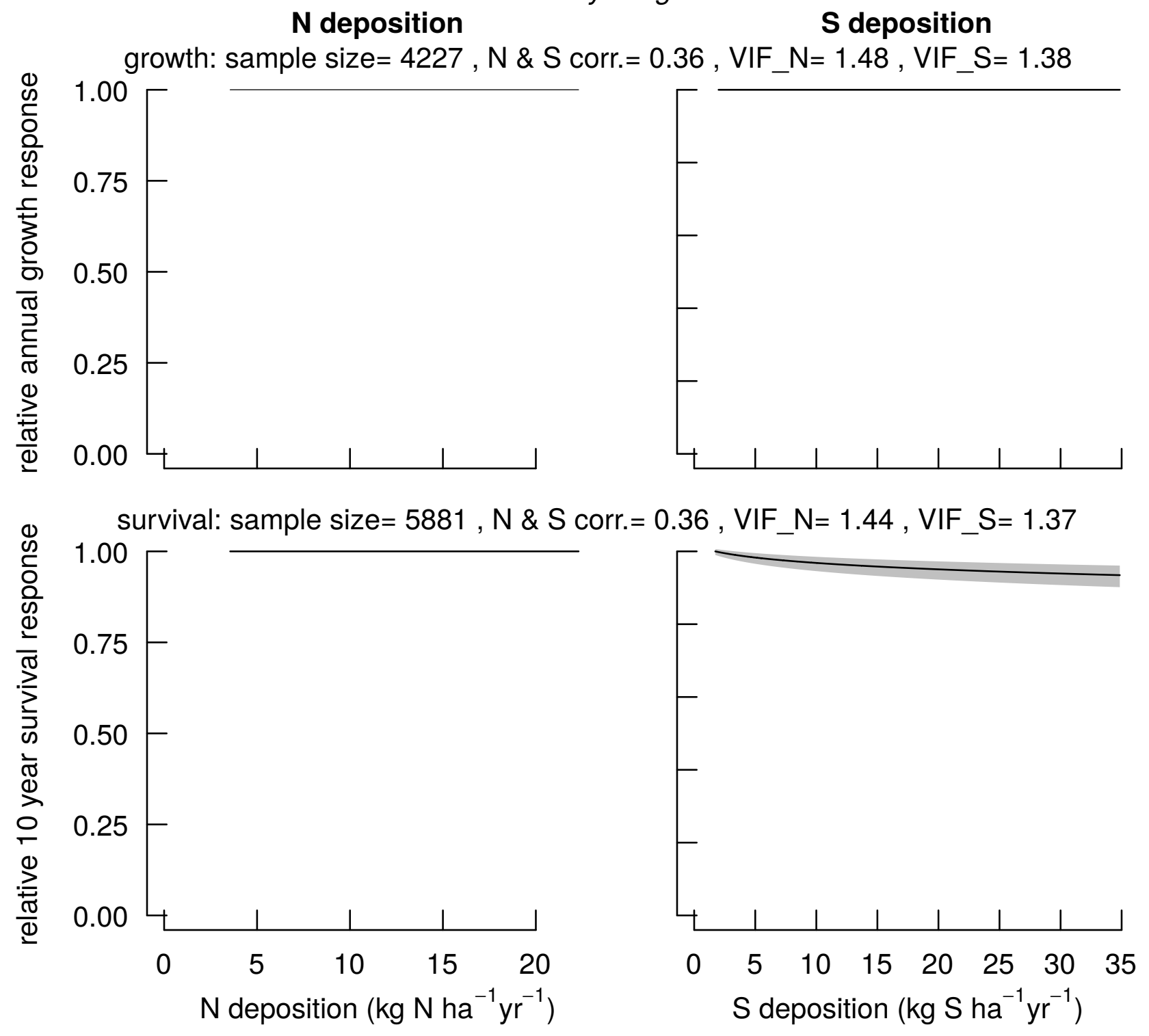




\section{sourwood \\ Oxydendrum arboreum}

N deposition

S deposition
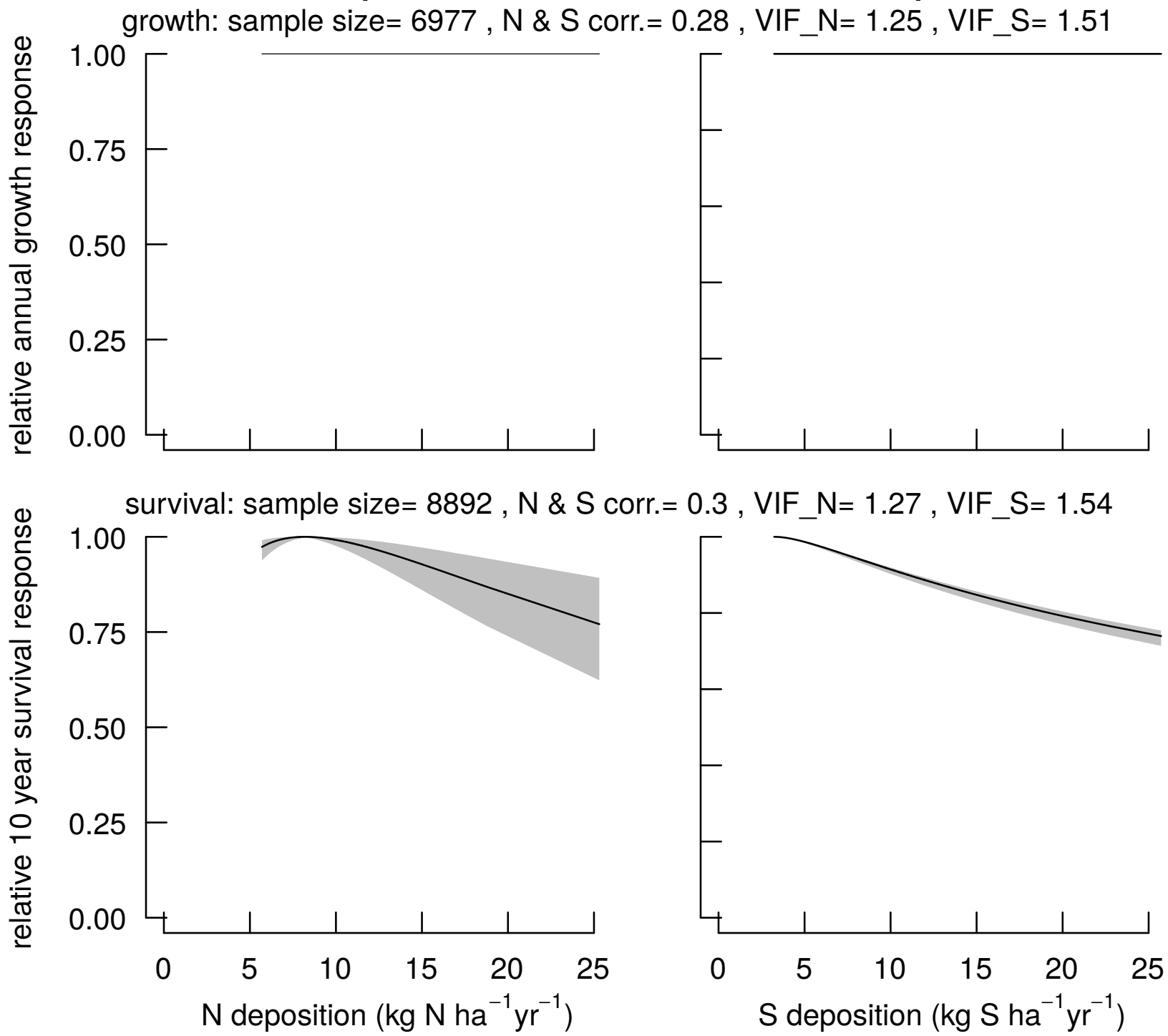
American sycamore

Platanus occidentalis

\section{N deposition}

$S$ deposition
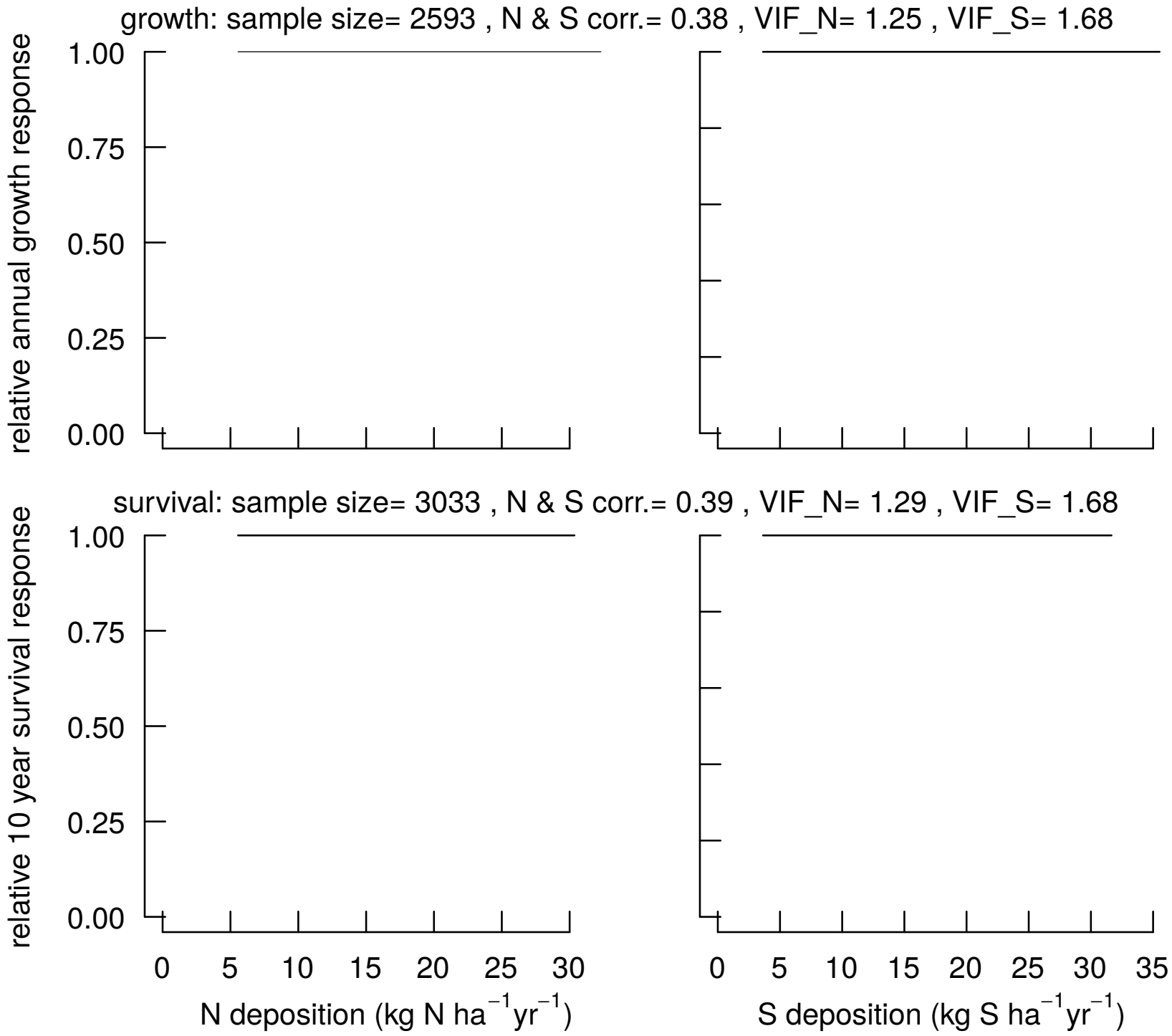


\section{balsam poplar}

N deposition

Populus balsamifera

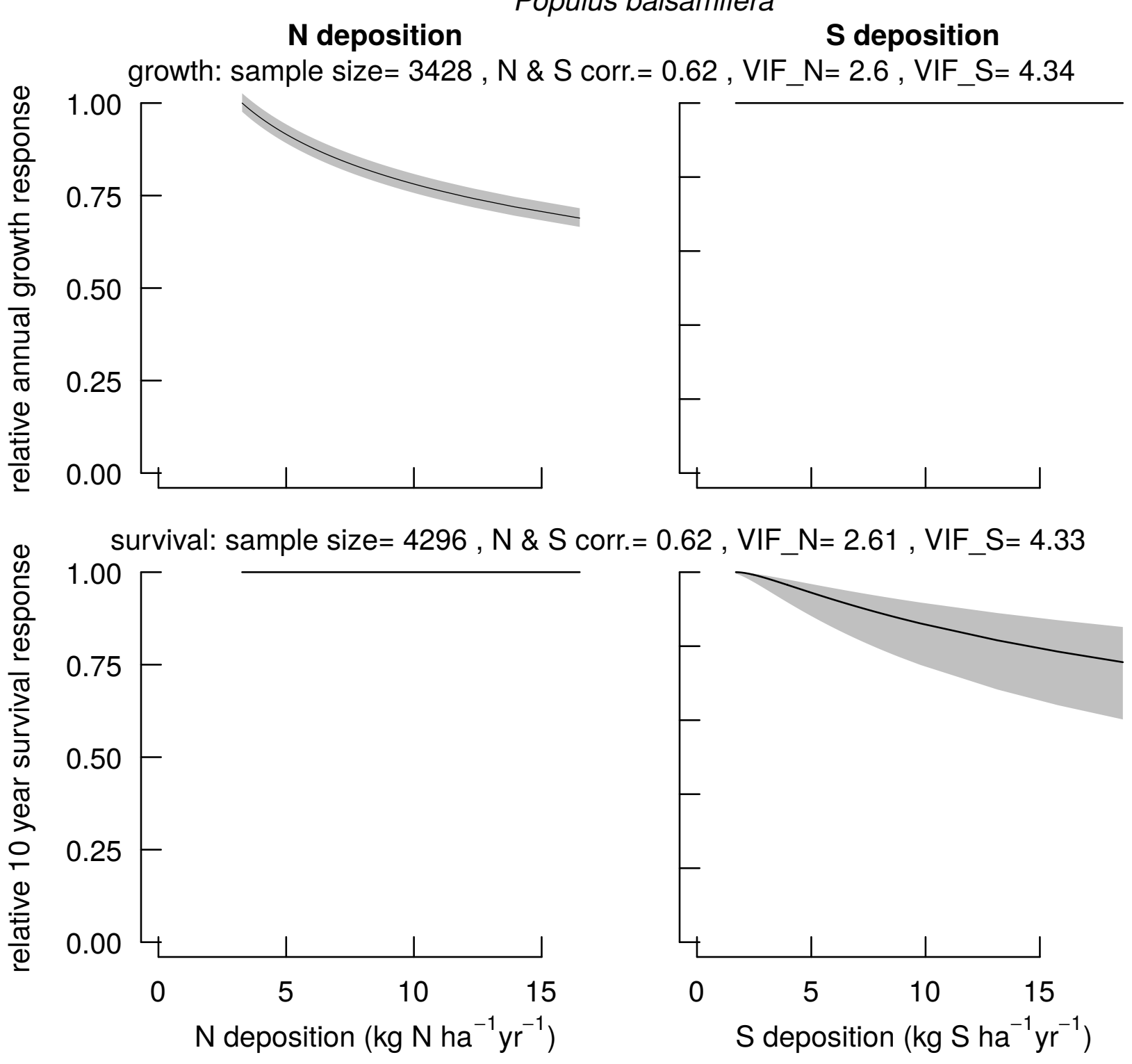




\section{bigtooth aspen}

Populus grandidentata

\section{$\mathrm{N}$ deposition}

\section{$S$ deposition}
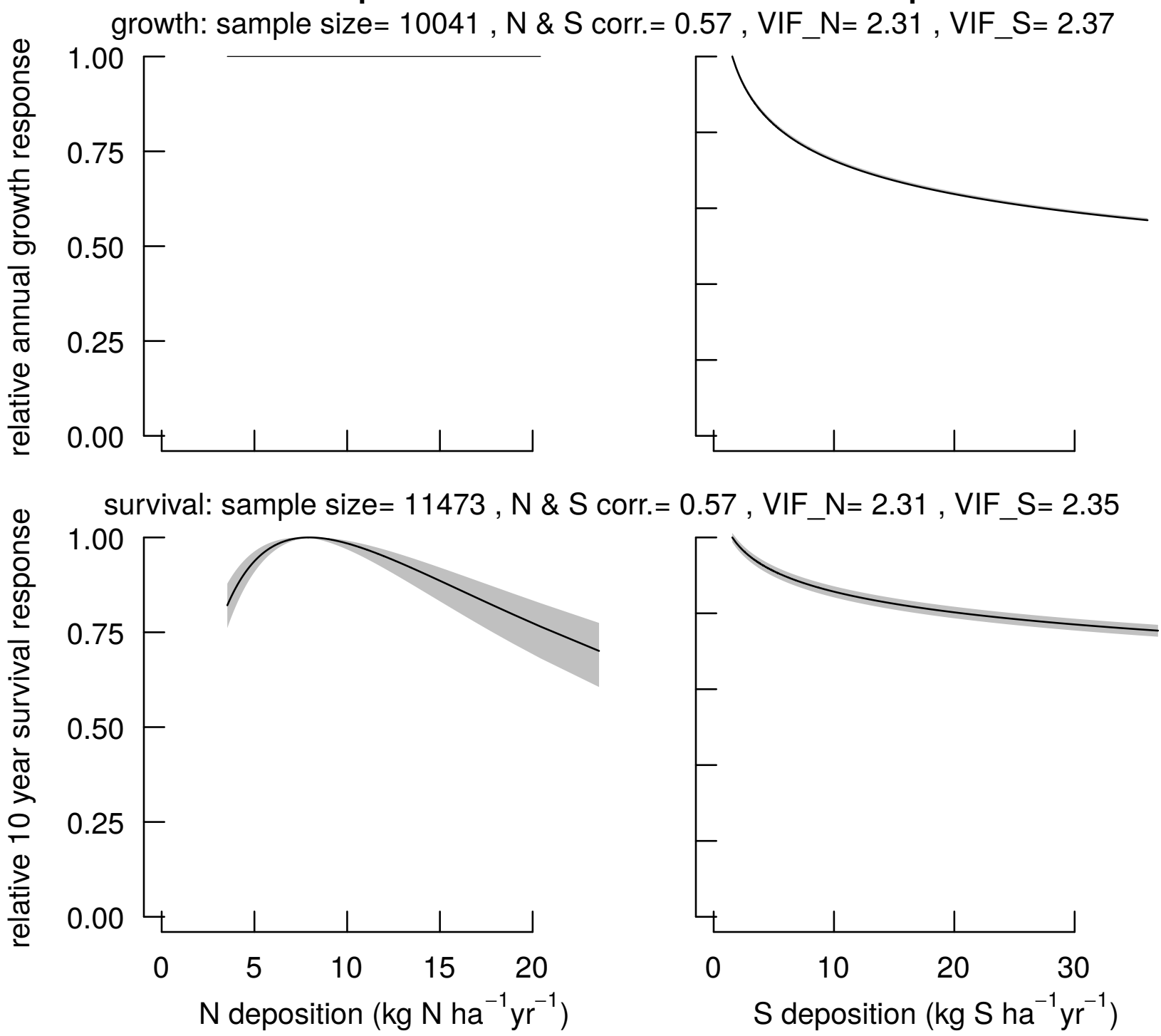


\section{quaking aspen}

N deposition

Populus tremuloides

\section{$\mathrm{S}$ deposition}

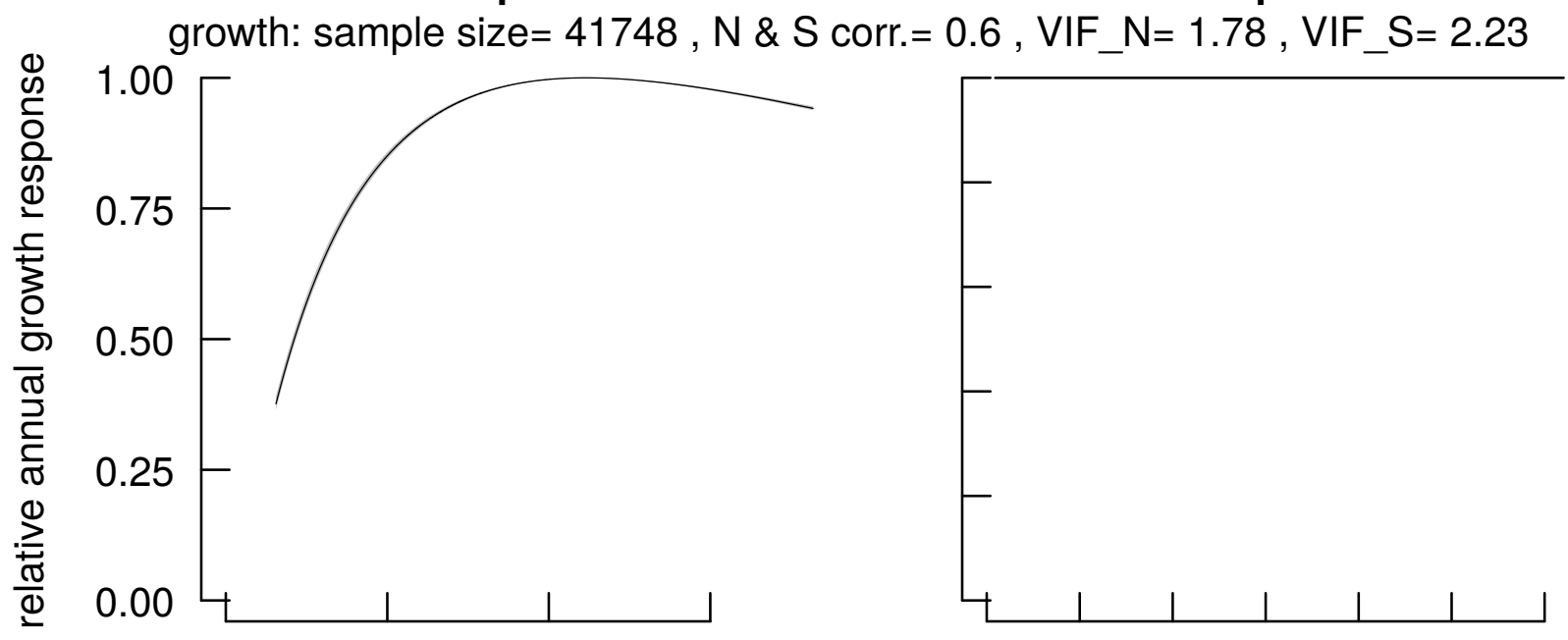

(1) survival: sample size $=51305, \mathrm{~N} \& \mathrm{~S}$ corr. $=0.61, \mathrm{VIF} \mathrm{N}=1.78, \mathrm{VIF} S \mathrm{~S}=2.26$

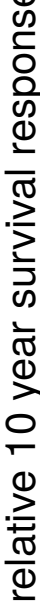
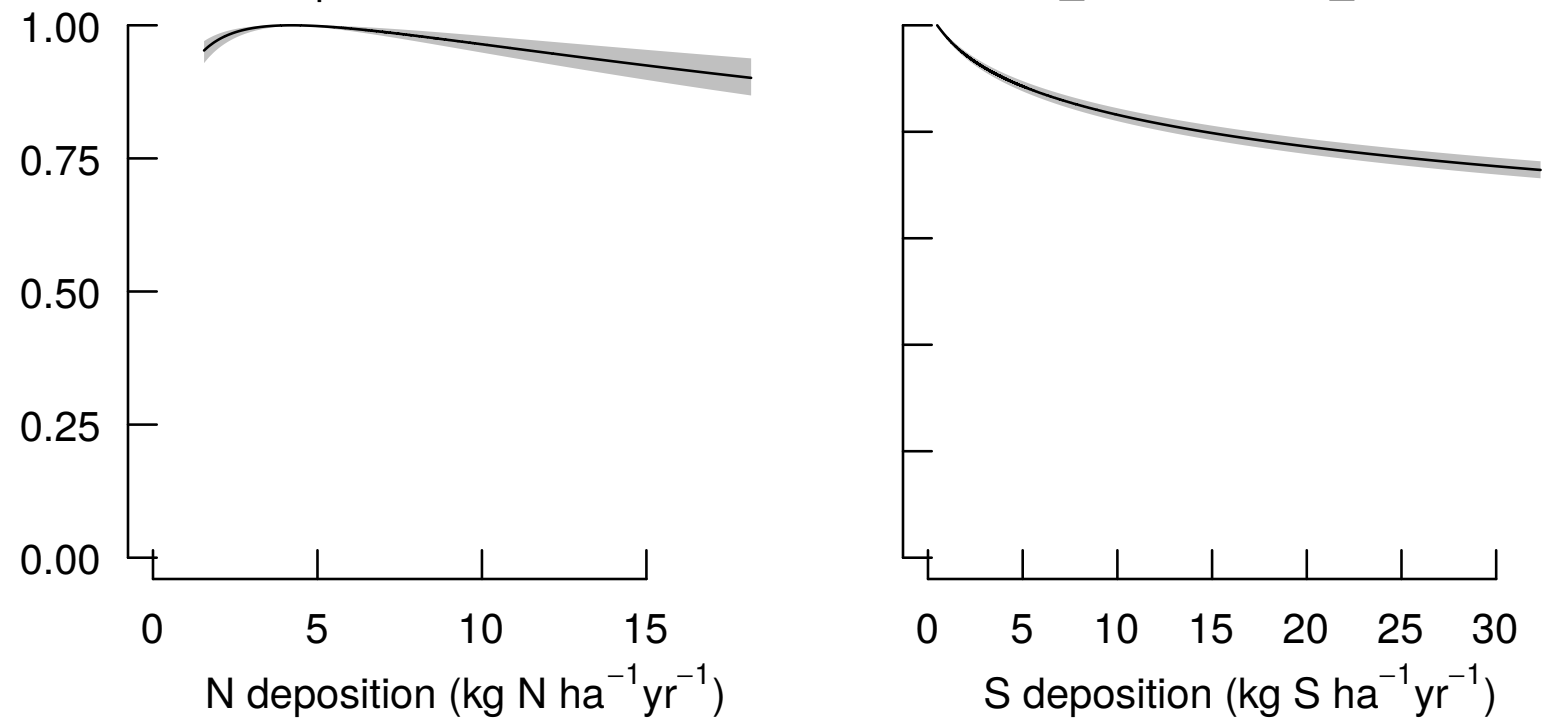


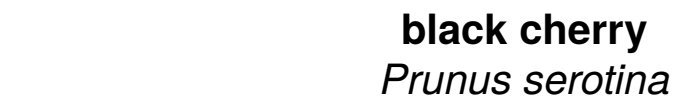

$\mathrm{N}$ deposition

Prunus serotina

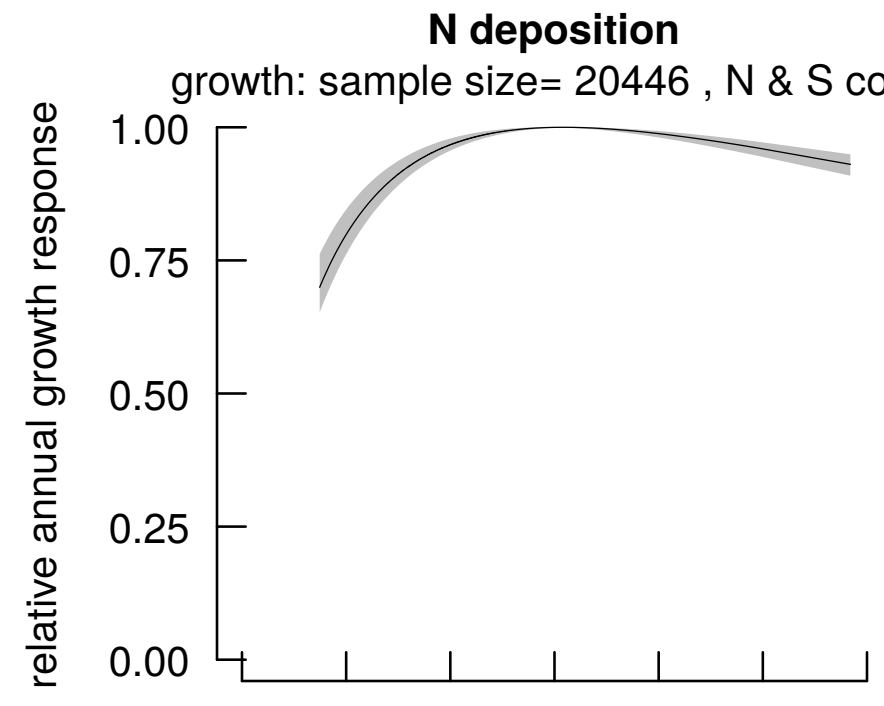

$S$ deposition
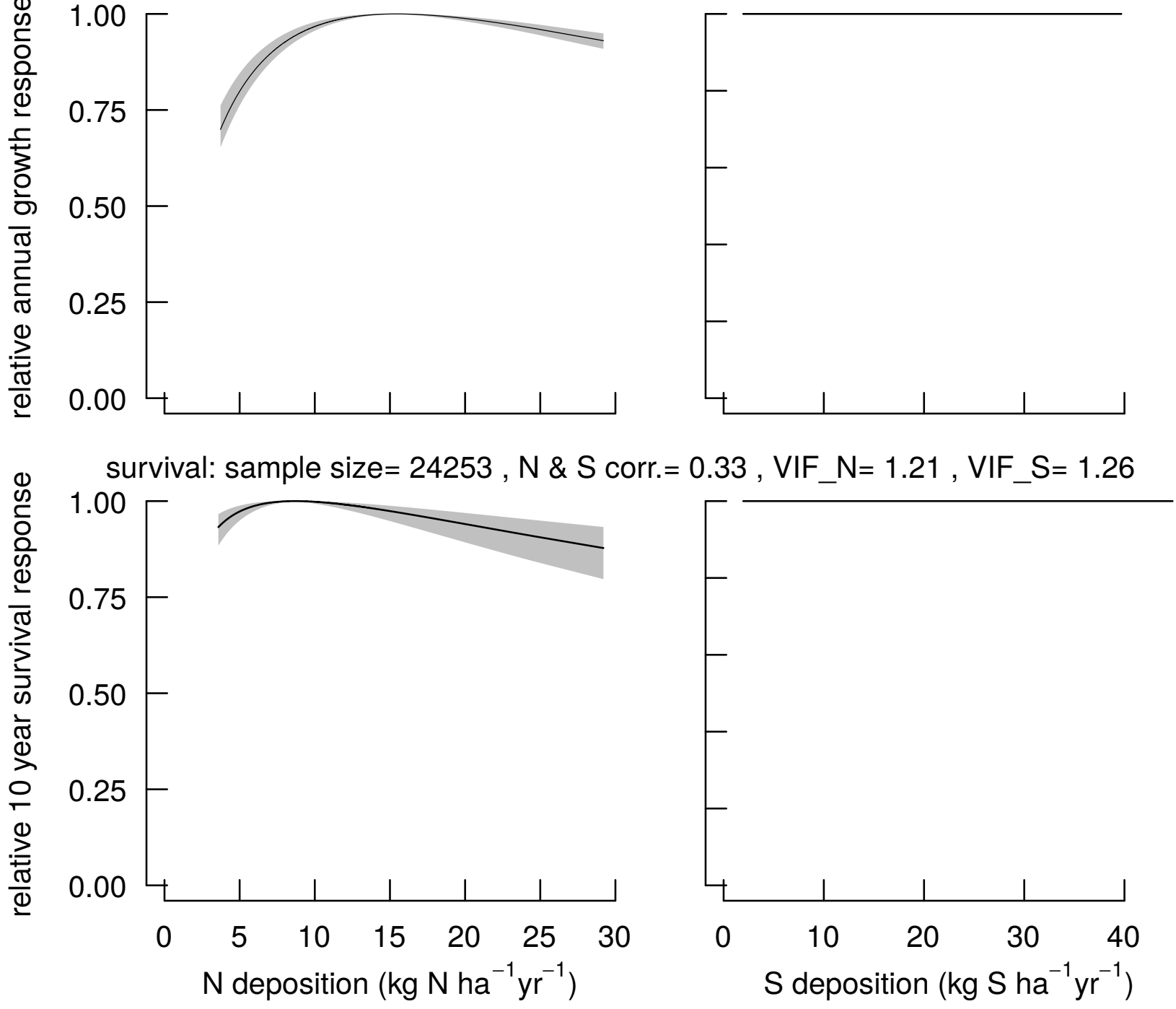


\section{white oak}

$\mathbf{N}$ deposition

Quercus alba

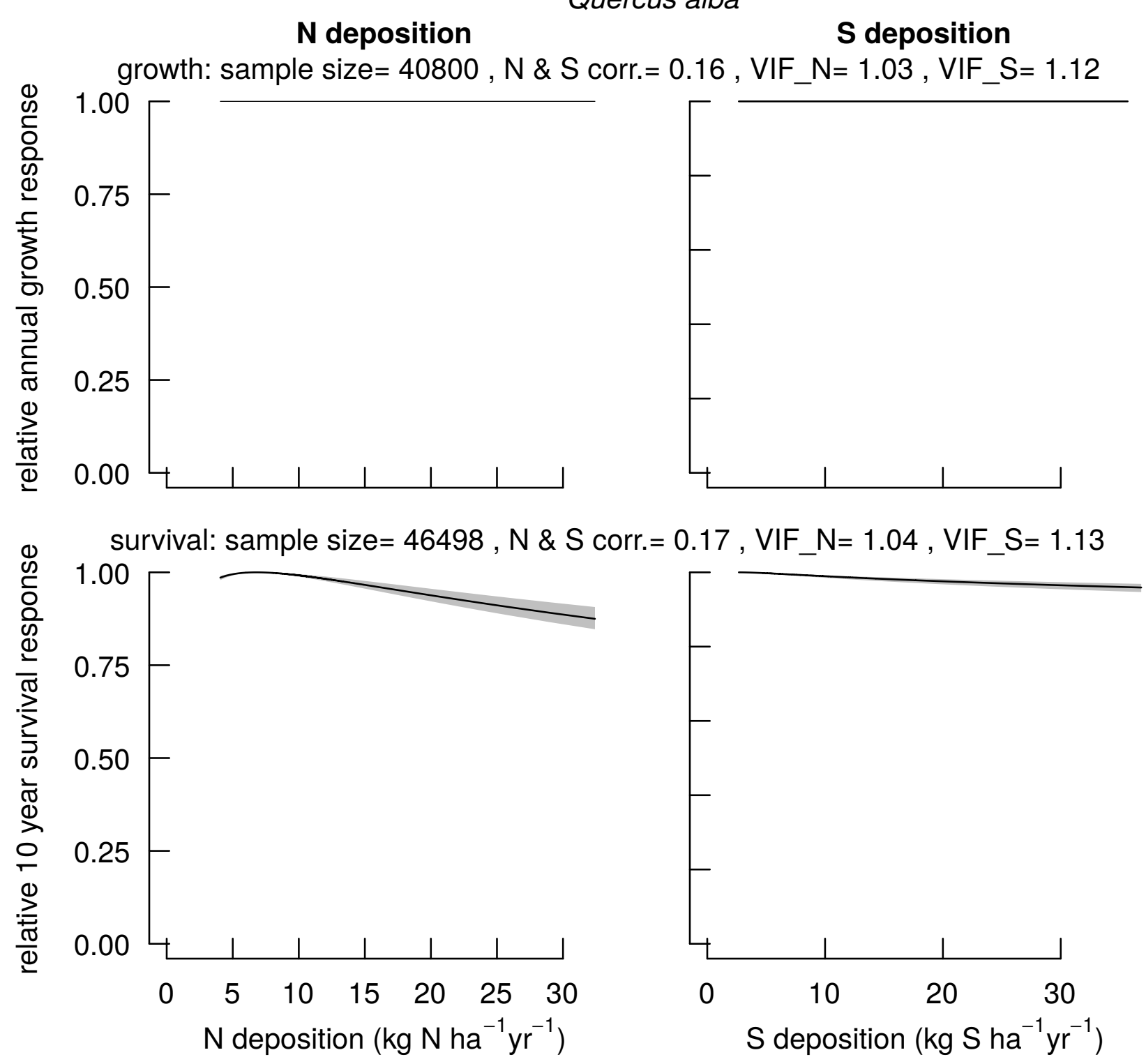




\section{canyon live oak}

N deposition

Quercus chrysolepis

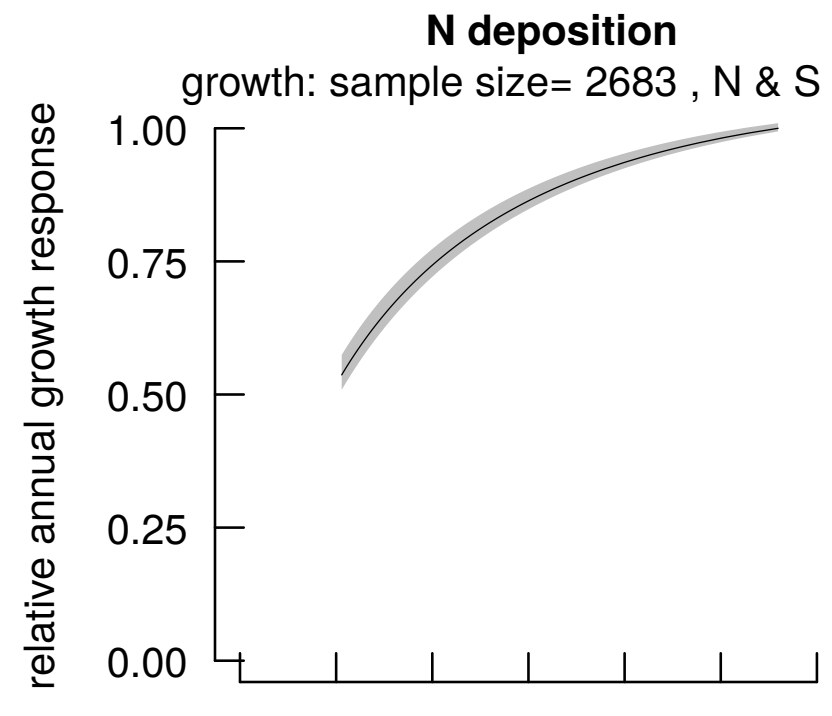

$S$ deposition
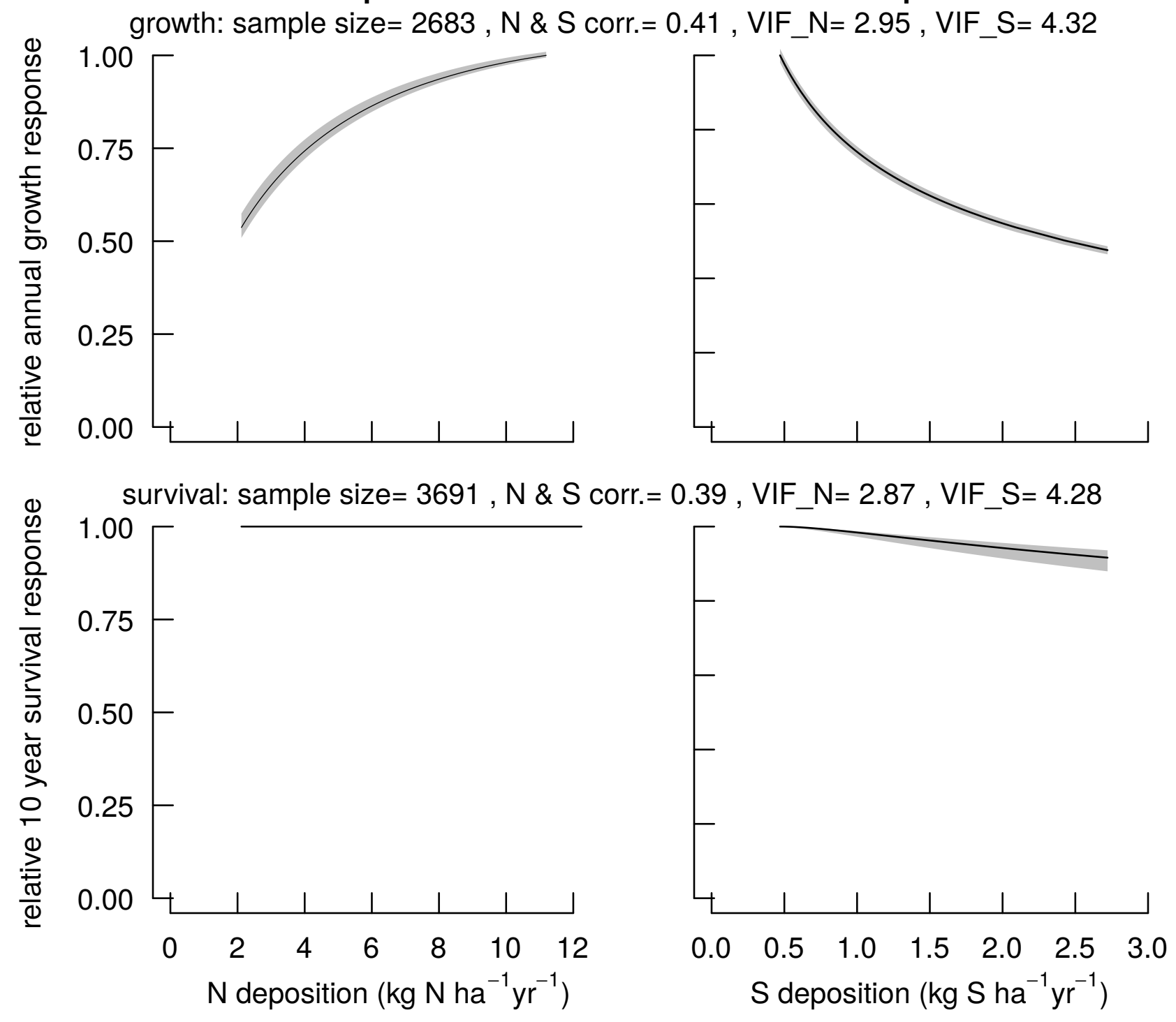


\section{scarlet oak}

N deposition

Quercus coccinea
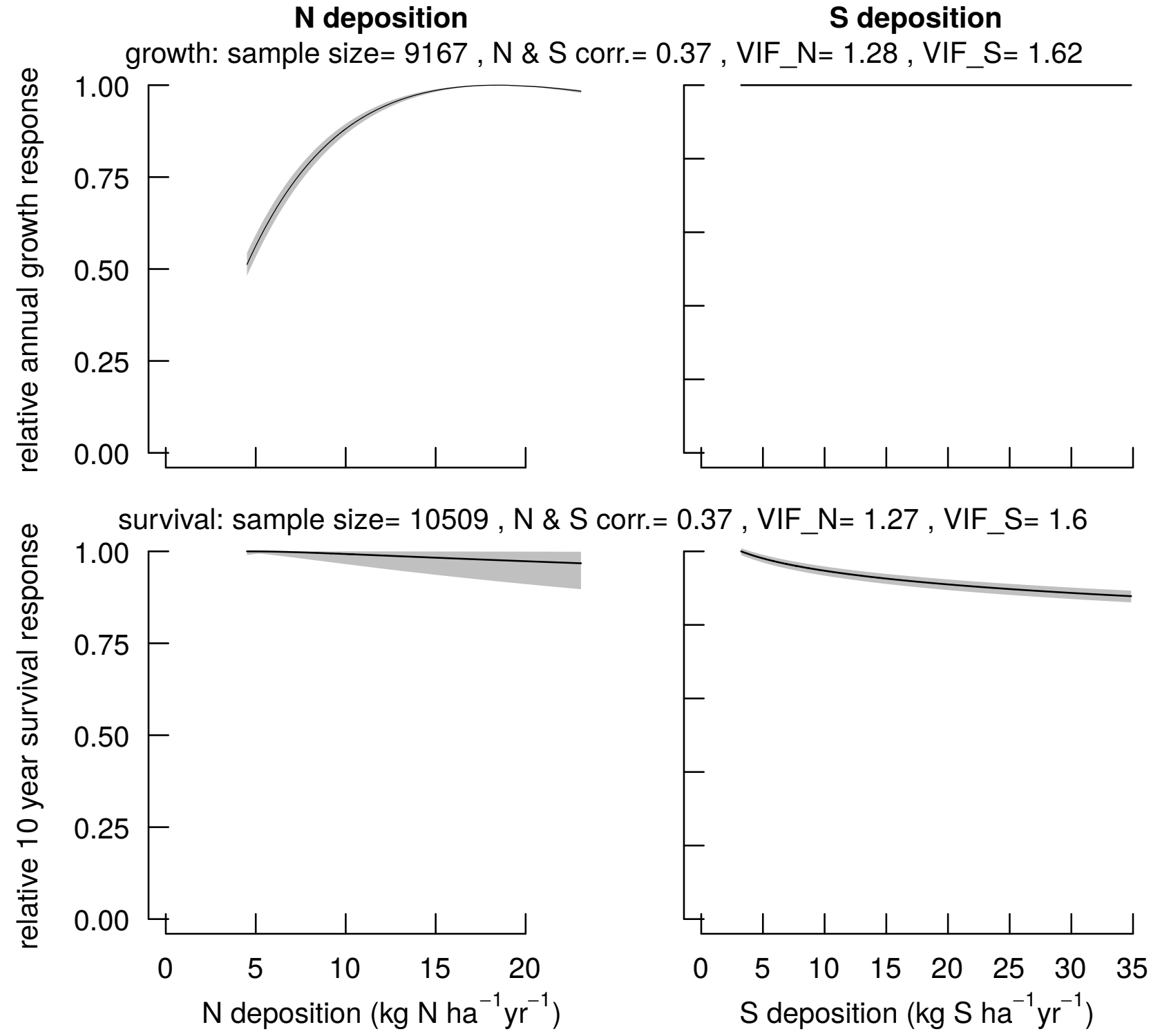

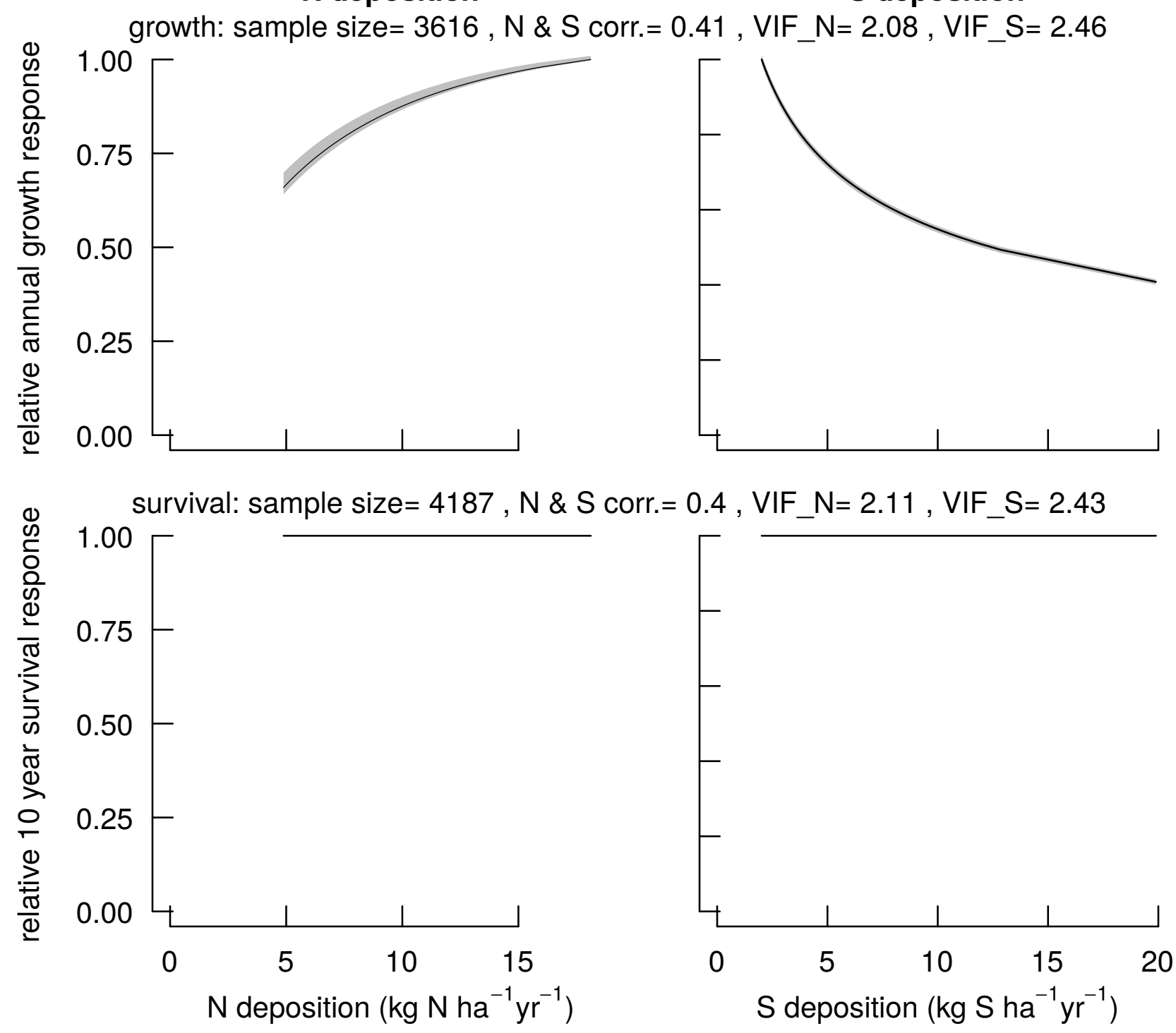


\section{southern red oak \\ Quercus falcata}

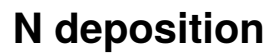

S deposition
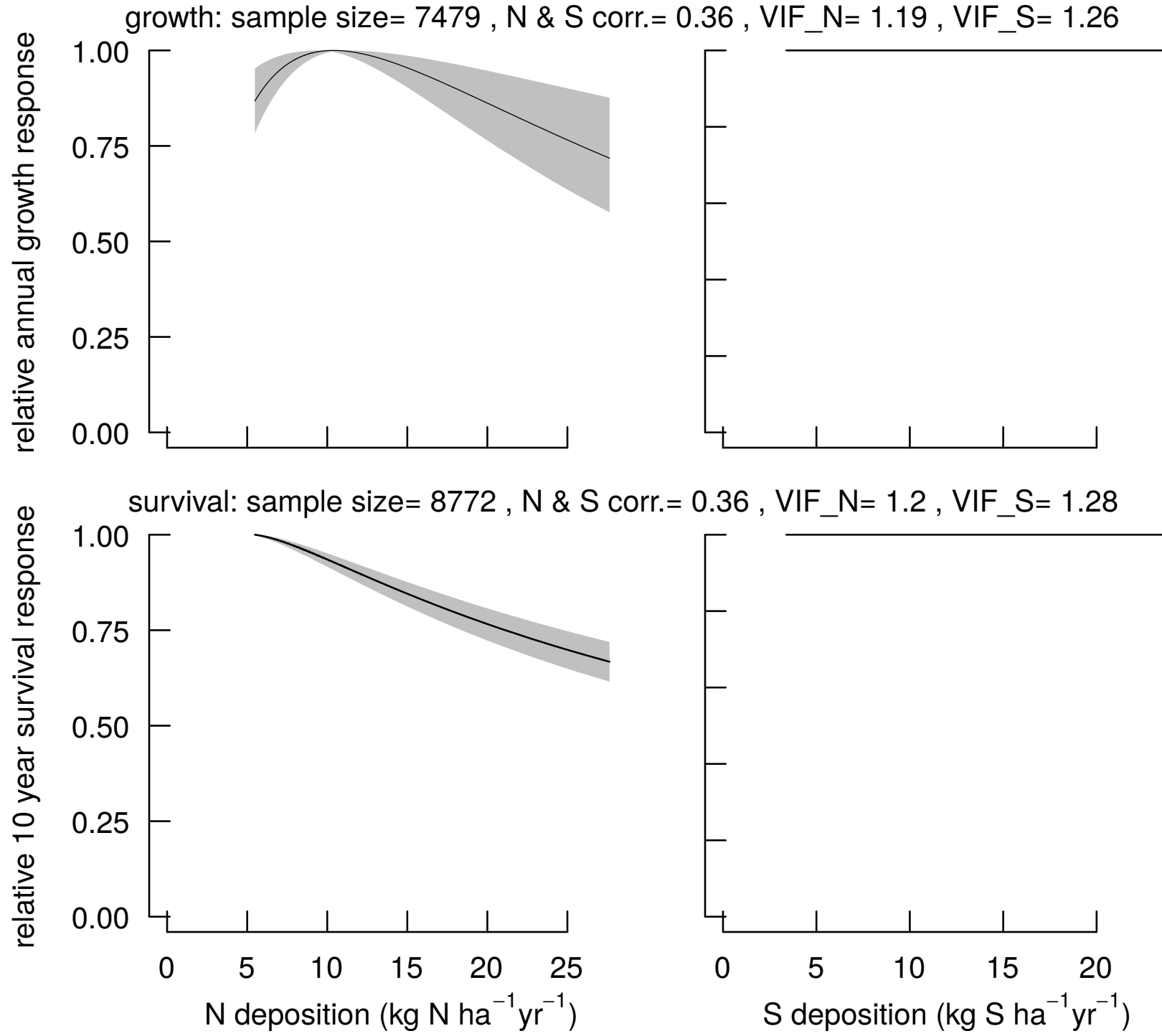
laurel oak

Quercus laurifolia

\section{N deposition}

$S$ deposition
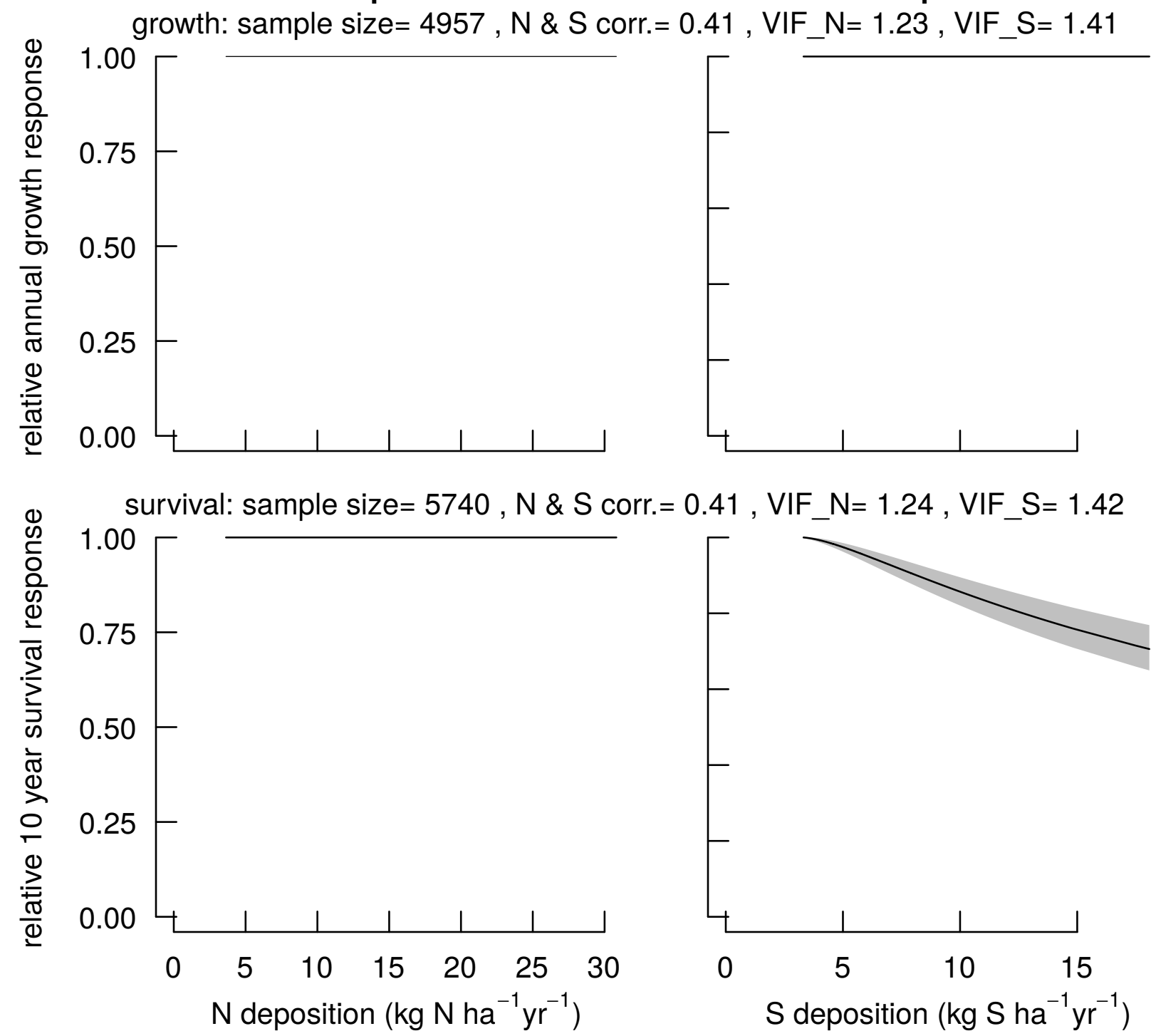


\section{bur oak}

Quercus macrocarpa

\section{N deposition \\ $\mathrm{S}$ deposition}
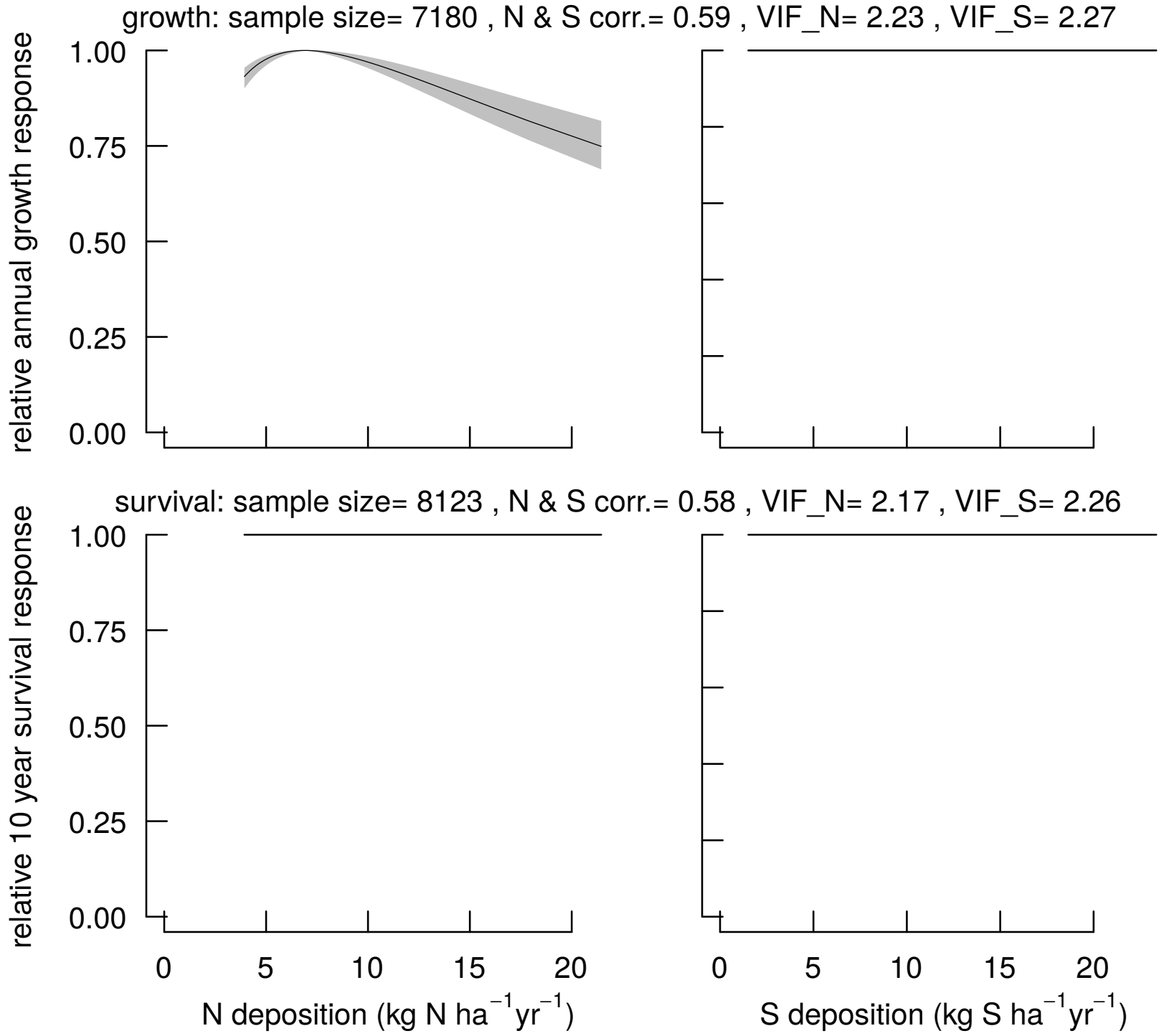


\section{chinkapin oak}

N deposition

Quercus muehlenbergii
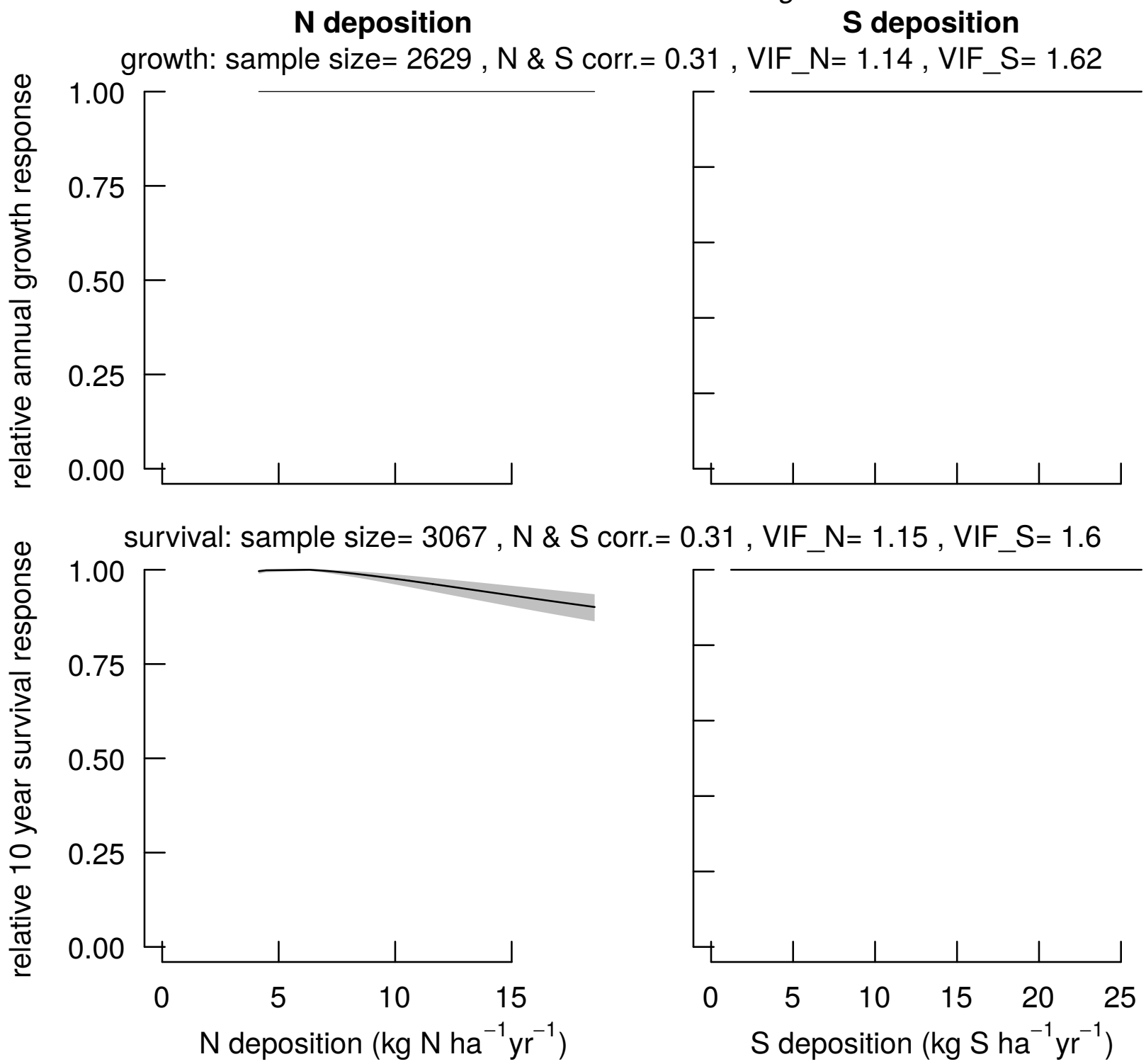


\section{water oak \\ Quercus nigra}

N deposition

$\mathbf{S}$ deposition
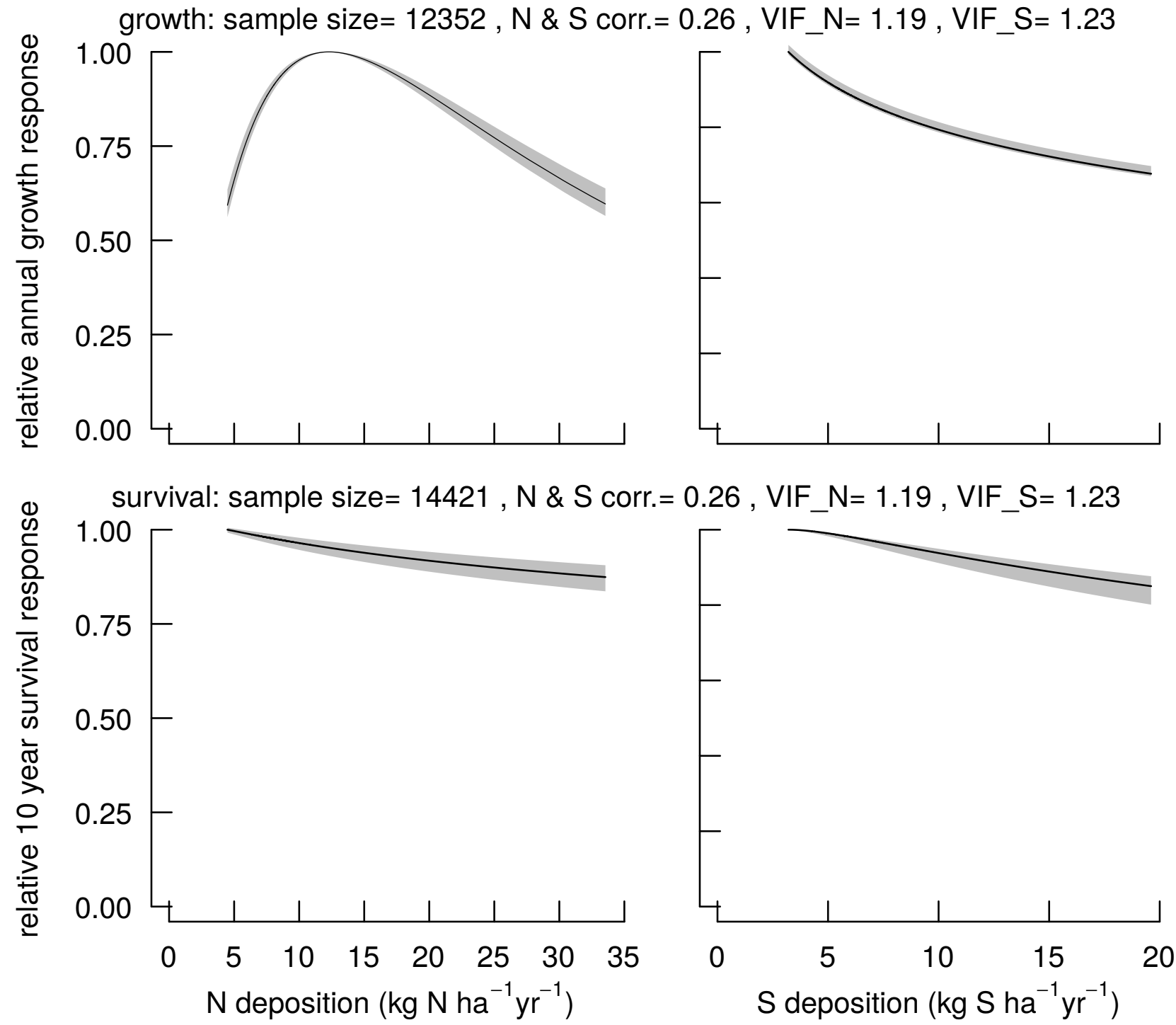


\section{willow oak}

$\mathbf{N}$ deposition

Quercus phellos

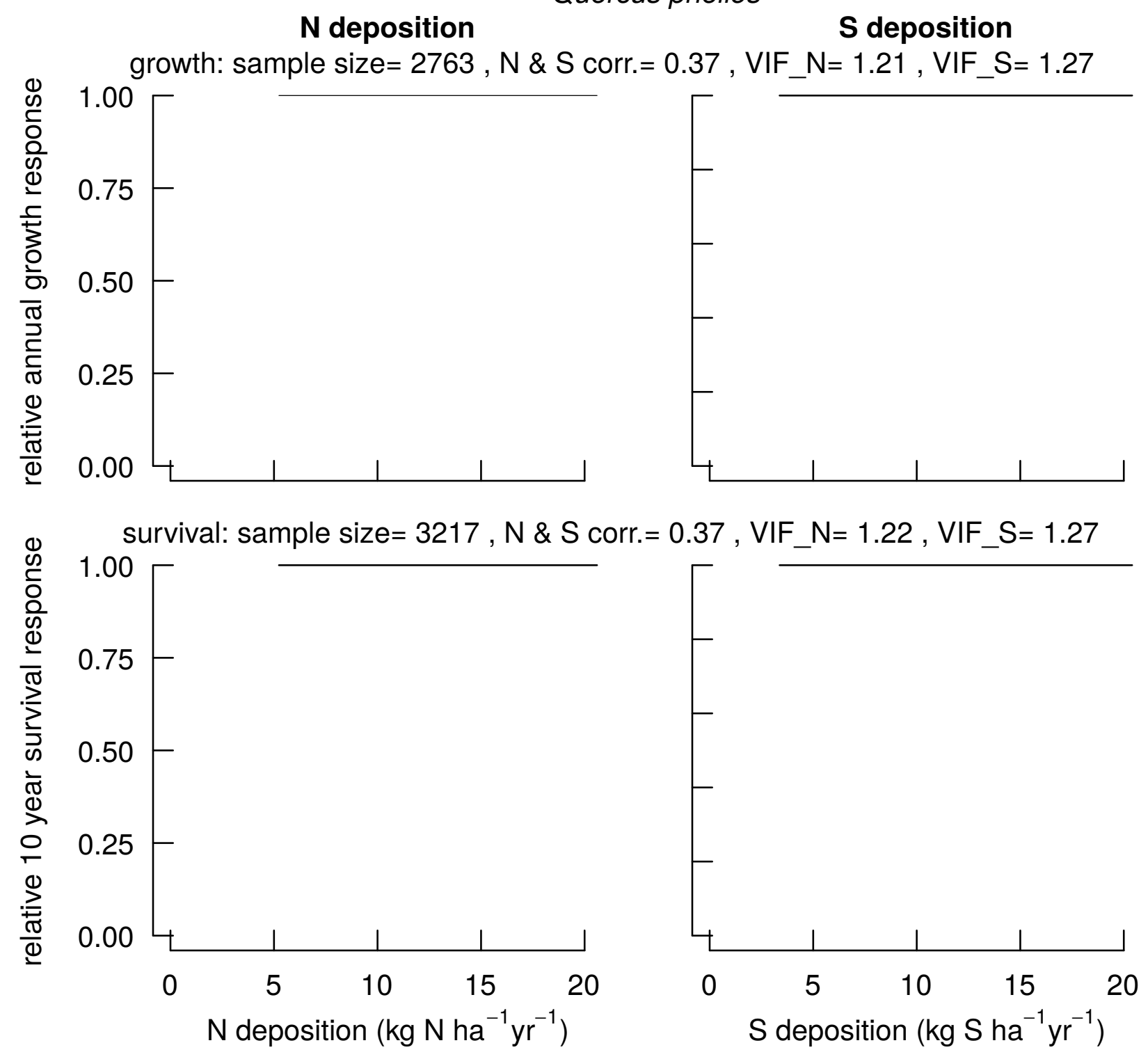




\section{chestnut oak}

N deposition

Quercus prinus

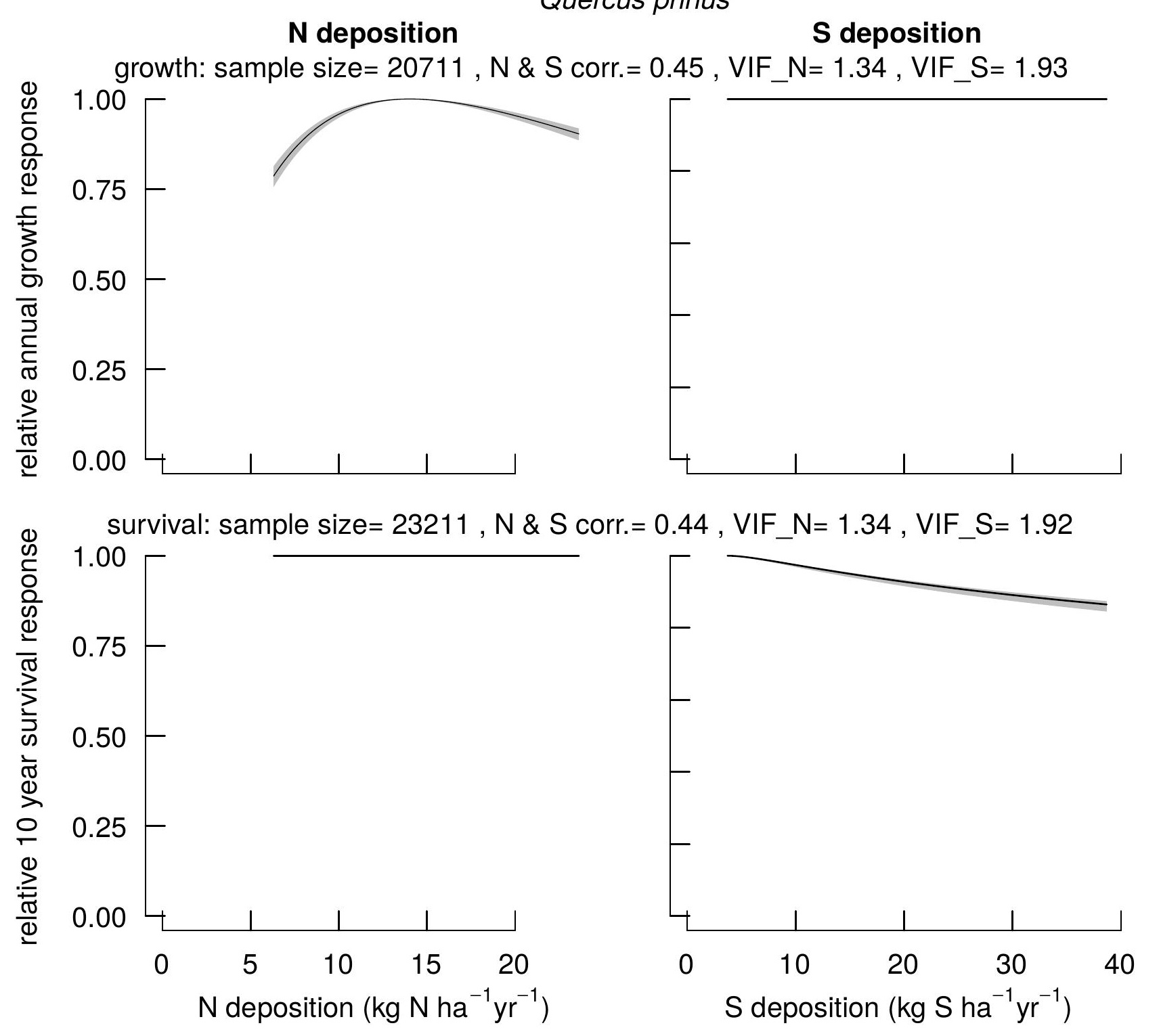




\section{northern red oak}

$\mathbf{N}$ deposition

Quercus rubra

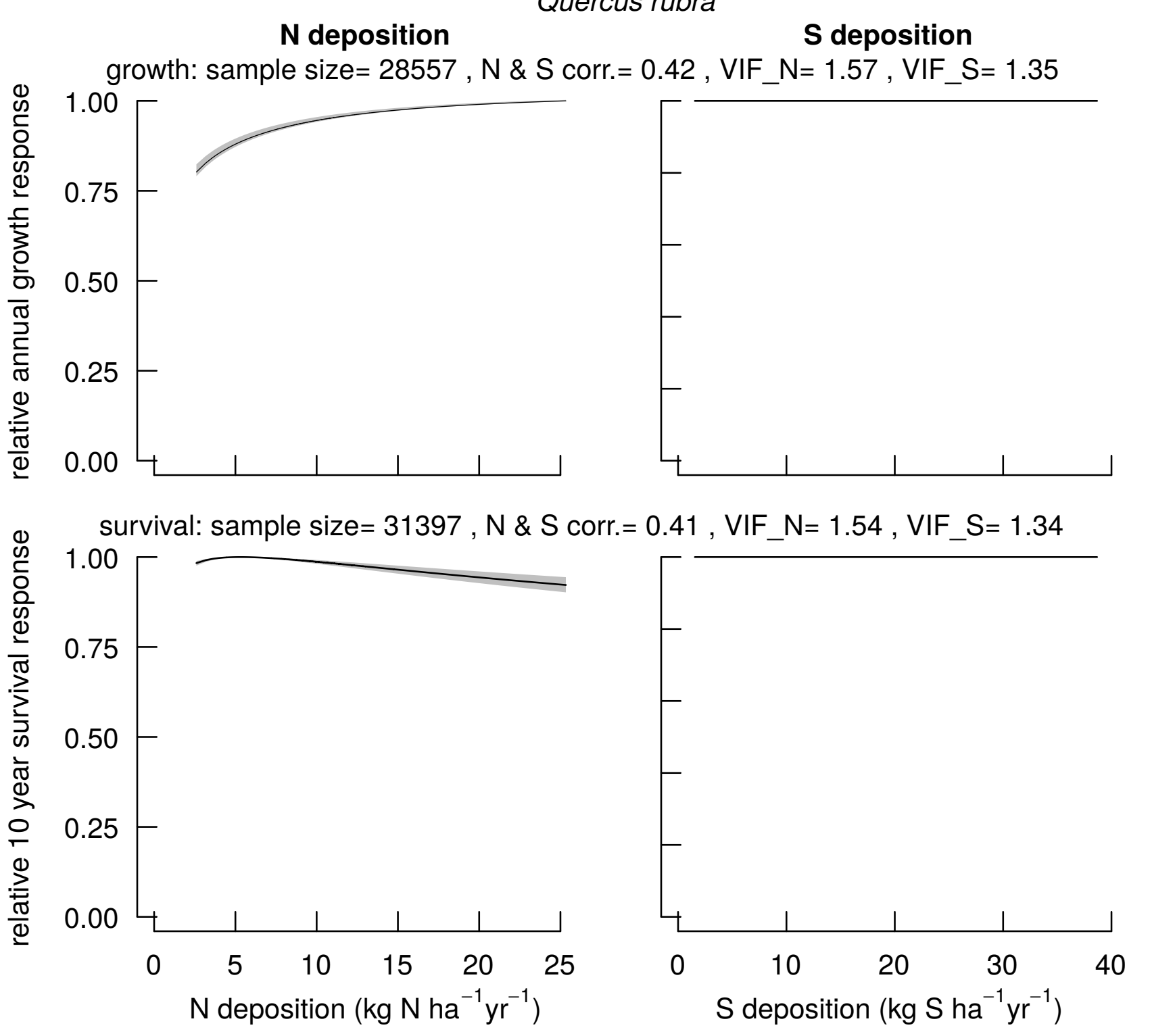




\section{post oak \\ Quercus stellata}

N deposition

$\mathbf{S}$ deposition
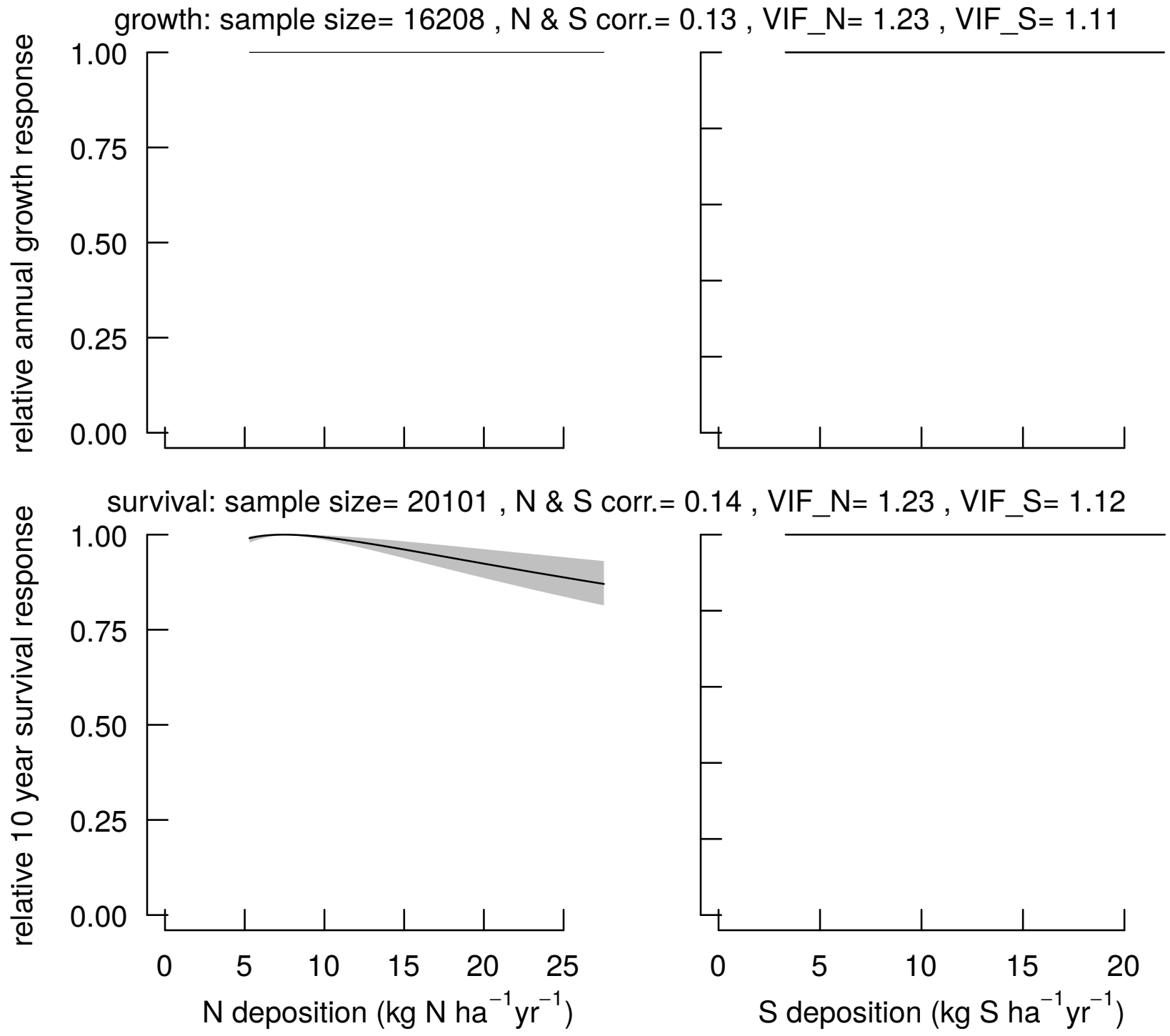


\section{black oak}

N deposition

Quercus velutina

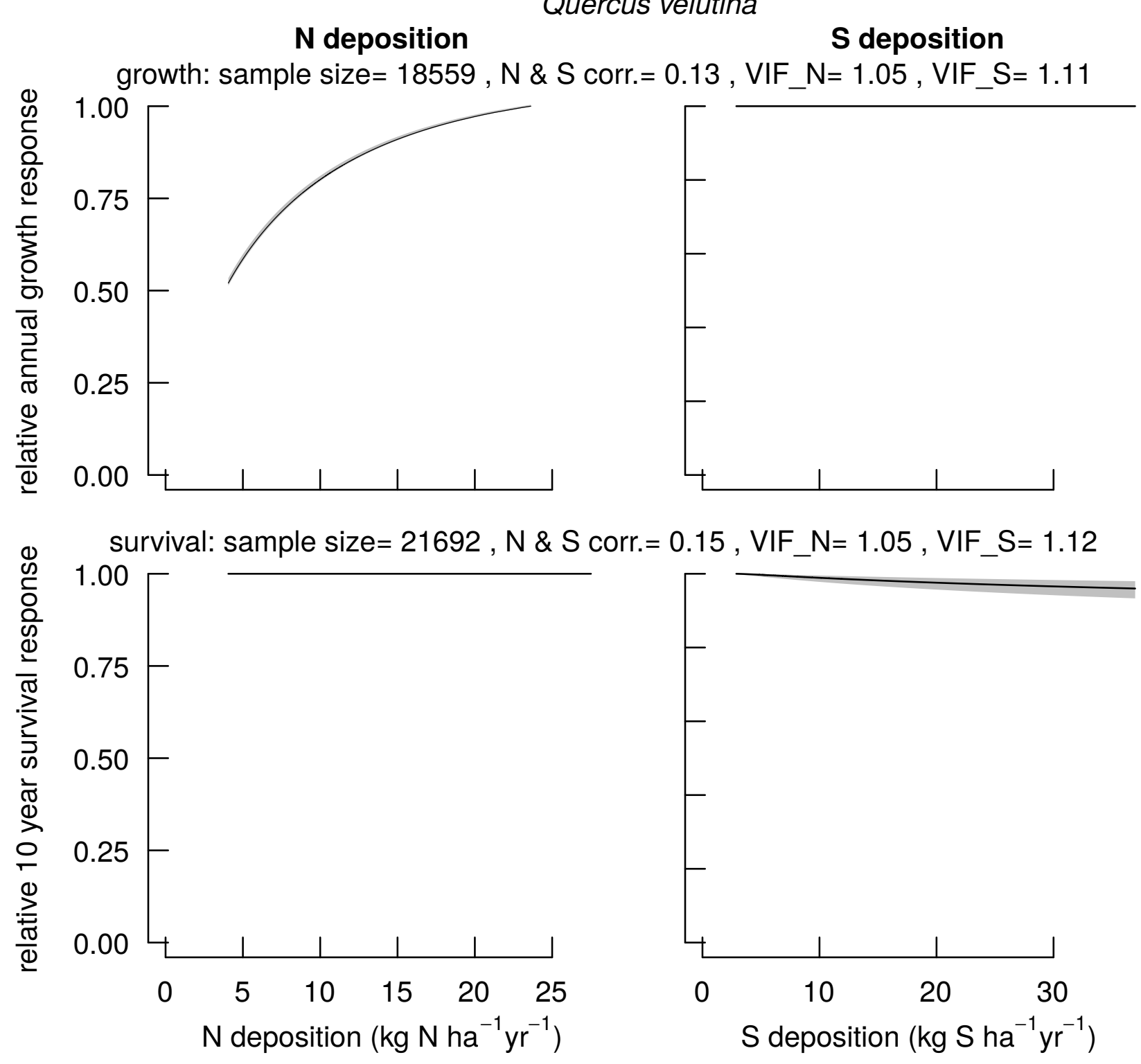




\section{black locust}

Robinia pseudoacacia

\section{N deposition \\ $S$ deposition}
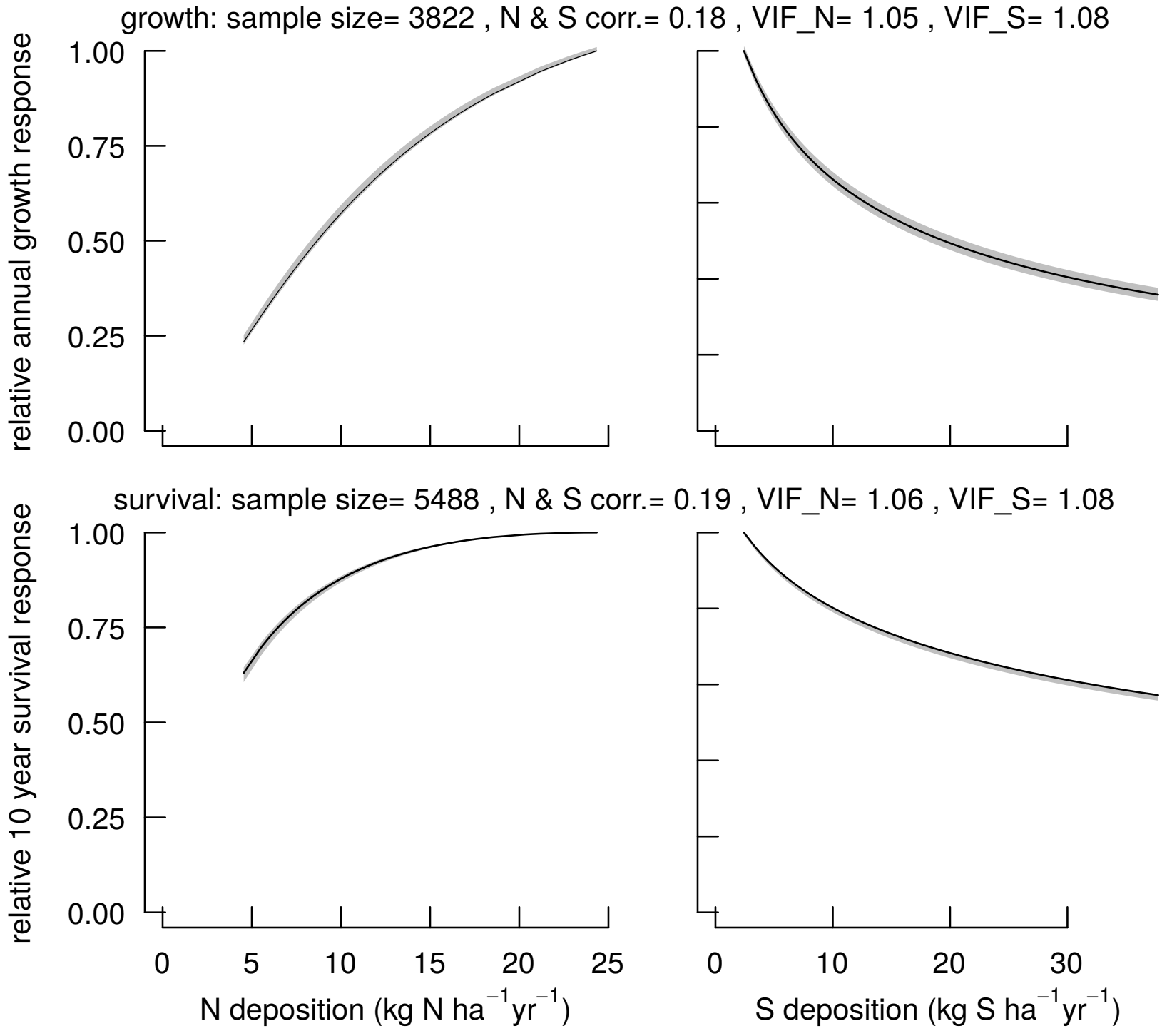


\section{black willow}

N deposition

Salix nigra
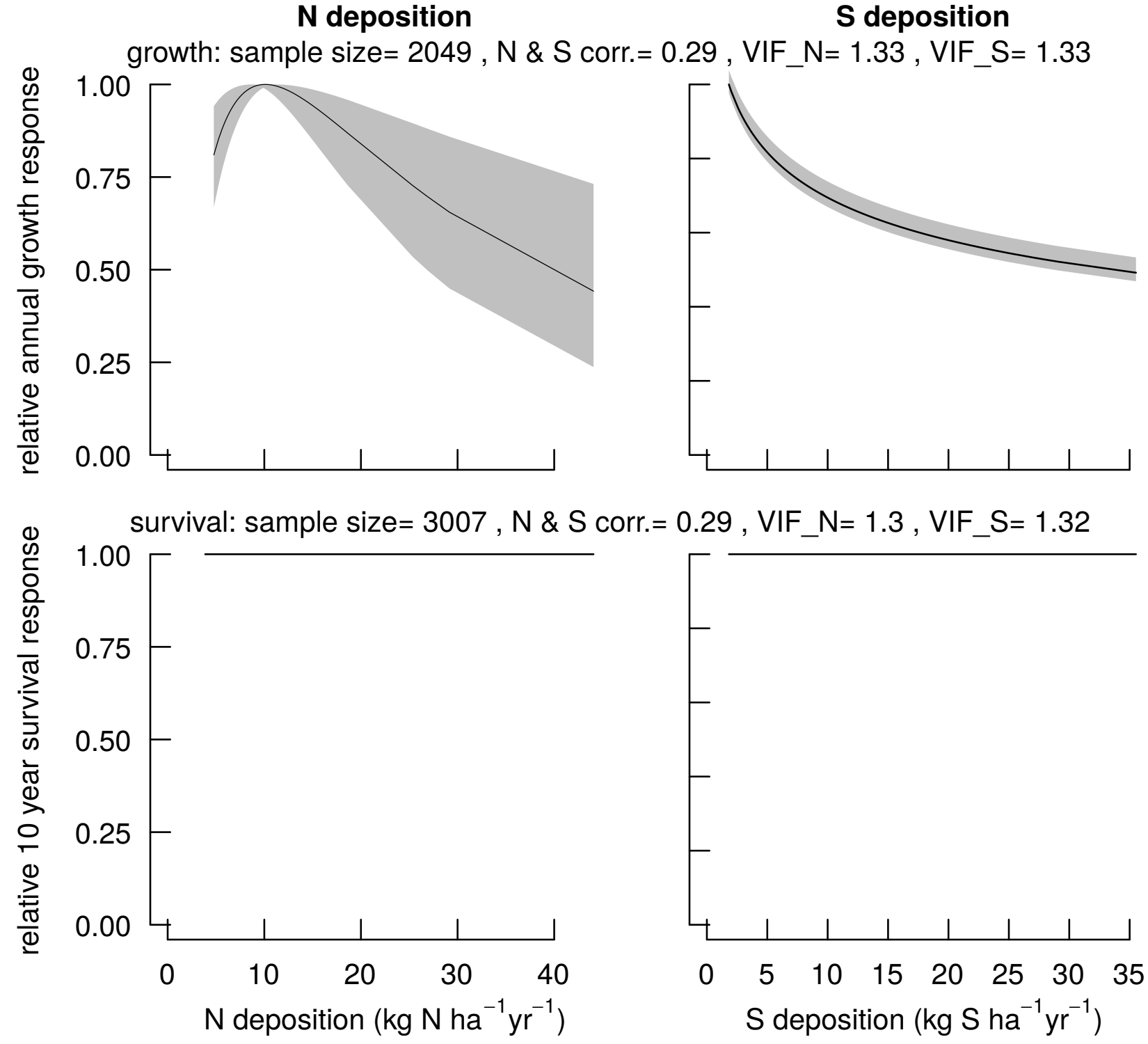


\section{sassafras}

Sassafras albidum

\section{N deposition}

$\mathrm{S}$ deposition

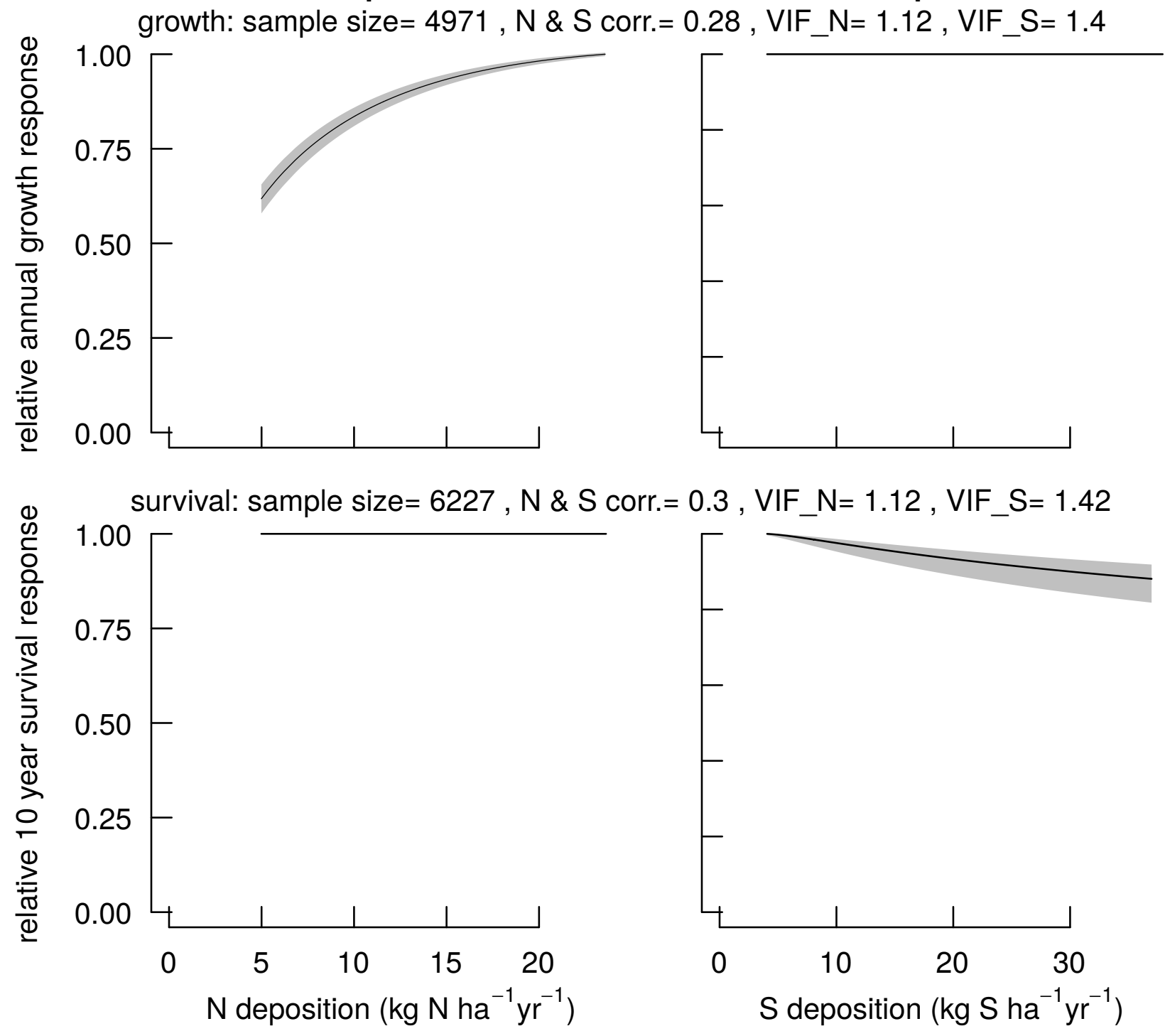




\section{American basswood}

Tilia americana

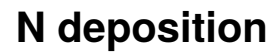

$\mathrm{S}$ deposition
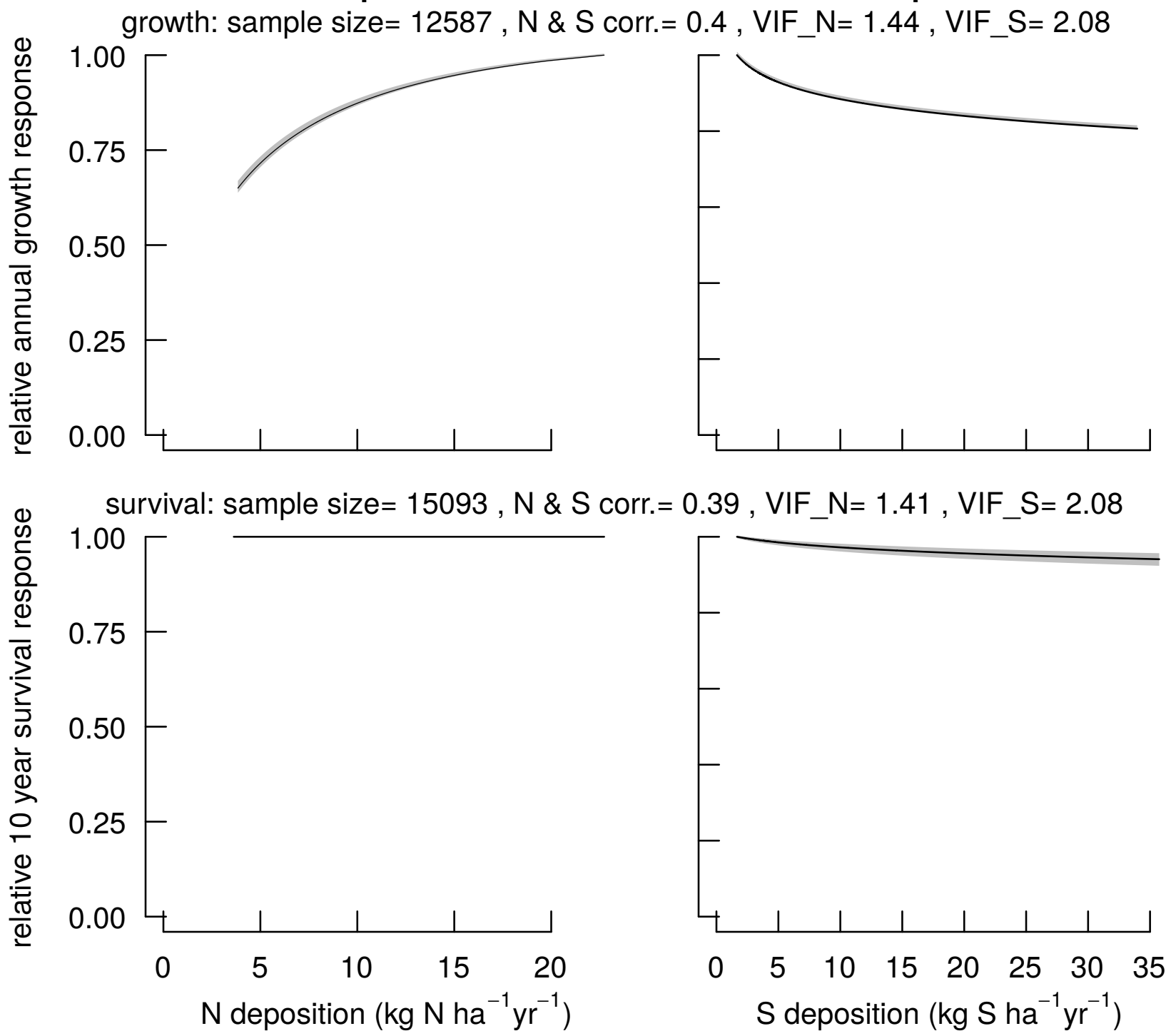


\section{winged elm}

$\mathbf{N}$ deposition

Ulmus alata
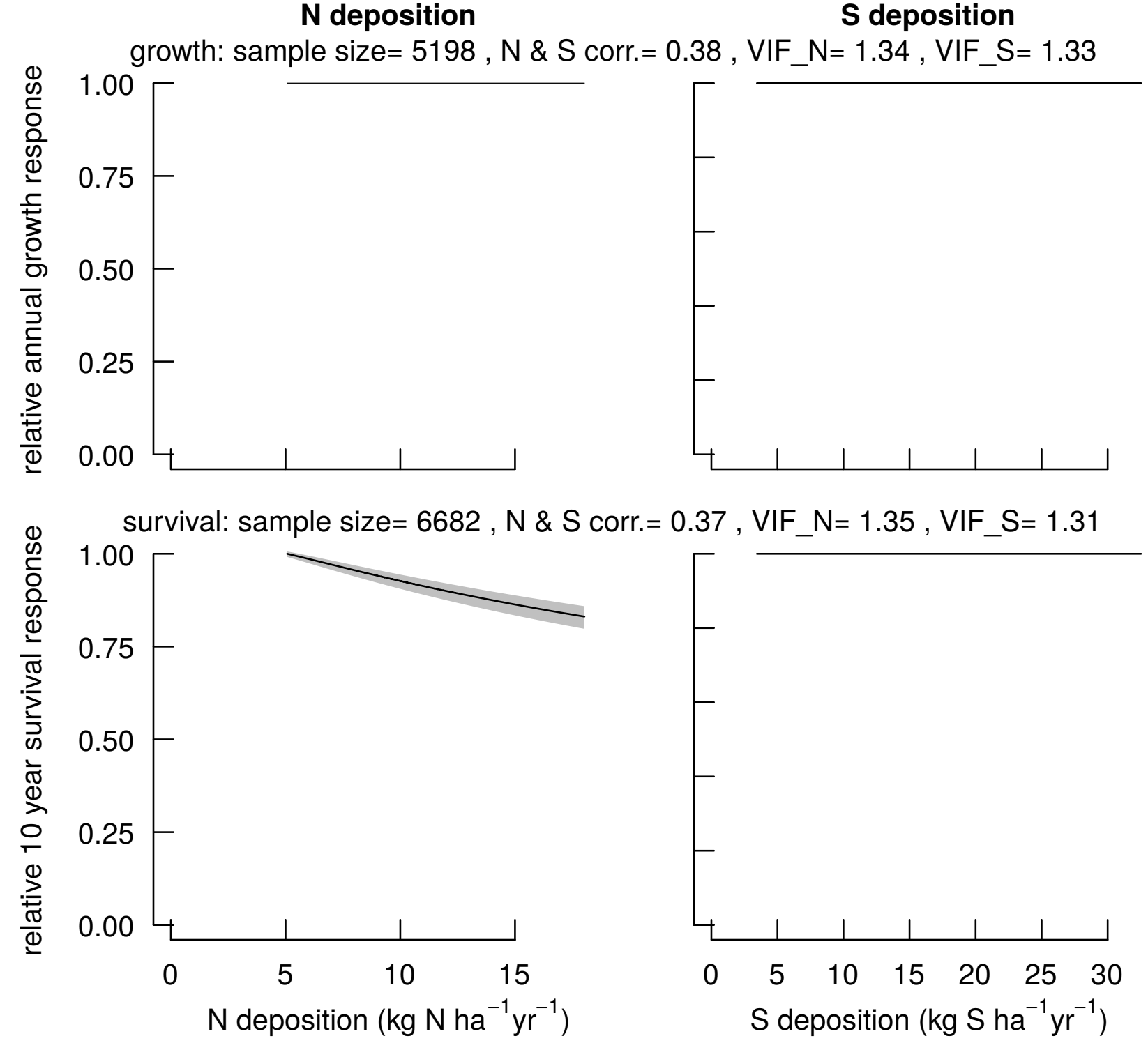
American elm

$\mathrm{N}$ deposition

Ulmus americana

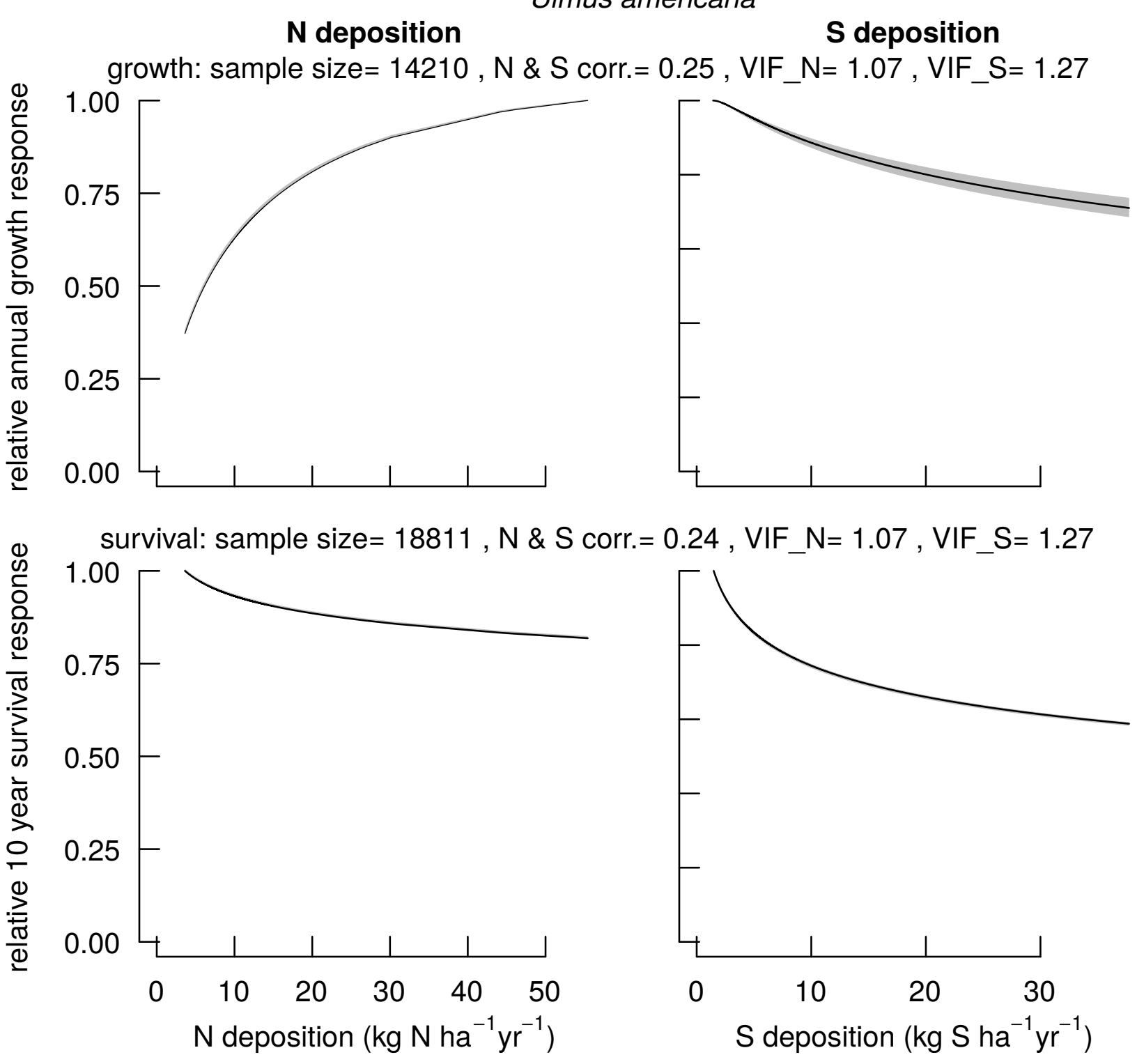


slippery elm

N deposition

Ulmus rubra

\section{$\mathbf{S}$ deposition}
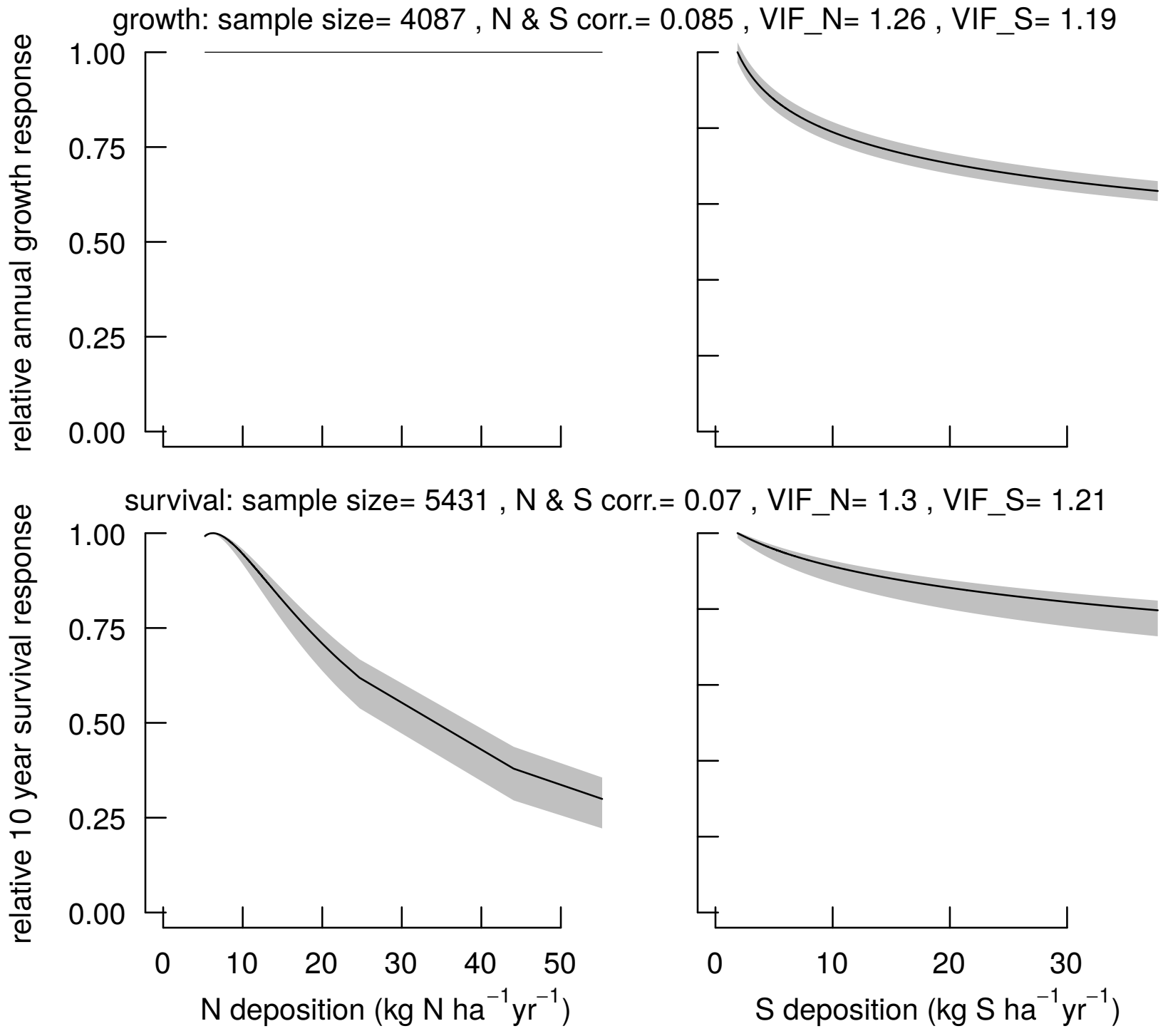